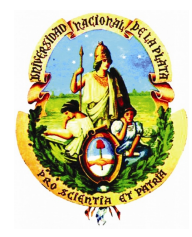

\title{
DETECCIÓN DE ESTRUCTURAS GEOLÓGICAS POTENCIALMENTE EN EQUILIBRIO ISOSTATICO A PARTIR DEL ANÁLISIS DE MODELOS GEOPOTENCIALES Y ANOMALÍAS DE BOUGUER
}

Tesis de Maestría

\section{CLAUDIA INFANTE}

Presentada ante la

Facultad de Ciencias Astronómicas y Geofísicas de la Universidad Nacional de La Plata como requisito para la obtención del Grado Académico de MAGÍSTER EN GEOMÁTICA

Directora de Tesis: Dra. Claudia Tocho Codirector de Tesis: Dr. Daniel Del Cogliano

Jurado de Tesis: Dr. Mauricio Gende Dr. Luis Guarracino

Dra. Patricia Martinez

La Plata, 19 de Abril de 2013 
Dedico este trabajo a la memoria de mis padres 


\section{Agradecimientos}

Deseo expresar mi más sincero agradecimiento a la Dra. Claudia Tocho ${ }^{1}$ y Dr. Daniel Del Cogliano ${ }^{1}$ quienes aportaron la idea inicial del plan de trabajo de la presente Tesis.

En particular quiero agradecer a Dra. Claudia Tocho que como directora supo con excelencia académica, generosidad y entusiasmo permanente guiarme en todo el desarrollo de este trabajo. Gracias por su afecto, paciencia y voluntad de trabajo inquebrantable que apuntalaron en todo momento mi desempeño.

Asimismo quiero expresar mi profundo agradecimiento al Dr. Daniel Del Cogliano quien desde el inicio de mis estudios de maestría alentó mi interés por la Geodesia en general y la Geodesia Física en particular, y orientó mi línea de estudio e investigación. Gracias por sus oportunas y enriquecedoras sugerencias, por su afecto y apoyo.

A la Ing. Lucrecia Galván² con quien compartimos todo un camino de formación, trabajo e investigación, e hicieron más amenas las horas dedicadas a ello. Gracias por su afecto y apoyo incondicional.

Al Ing. Iber Goitea ${ }^{2}$ quien me entusiasmó a iniciar los estudios de maestría y con quien también compartimos el interés por la Geodesia y la Cartografía. Gracias por su afecto y apoyo incondicional.

A las autoridades de la Universidad Nacional de Santiago del Estero y de la Facultad de Ciencias Exactas y Tecnologías por el apoyo institucional.

A mis padres, que ya no están físicamente, quienes con amor me alentaron desde los inicios del postgrado y hoy lo hacen desde el cielo. A mis hermanos y amigos de siempre por su amor y apoyo constante.

A los docentes de cada uno de los cursos que conforman el Plan de Estudios de la Maestría en Geomática, por su dedicación y generosidad, por el afecto y calidez con que siempre me acogieron e hicieron más placenteros mis viajes a La Plata.

Finalmente agradezco a los Jurados de este trabajo, Dr. Mauricio Gende, Dr. Luis Guarracino y Dra. Patricia Martínez, por su dedicación en la revisión del texto y sus acertadas observaciones que enriquecieron la presente versión final.

\footnotetext{
${ }^{1}$ Universidad Nacional de La Plata

${ }^{2}$ Universidad Nacional de Santiago del Estero
} 


\section{Resumen}

El campo de gravedad terrestre nos proporciona información de las variaciones de densidad en el interior de la Tierra. Entre las aplicaciones del campo de gravedad en esta Tesis se analizan las anomalías de la gravedad y las ondulaciones del geoide como observables geofísicas.

En geofísica las anomalías de la gravedad se utilizan a escala local y regional para determinar la distribución de densidad en el interior terrestre y por lo tanto estudiar las estructuras geológicas a distintas profundidades.

El objetivo general de la presente Tesis consiste en identificar estructuras geológicas, en una región central de Argentina a partir del filtrado del modelo geopotencial EGM2008 y del cálculo de las anomalías de aire libre y las anomalías de Bouguer, y determinar a priori si están equilibradas ó no isostáticamente.

La zona de estudio comprende variada topografía, desde llanura al Este hasta montaña al Oeste, y mar en el extremo Sur-Este.

El estado de compensación de las estructuras geológicas se evalúa a partir del análisis de anomalías de aire libre y Bouguer calculadas a partir de datos terrestres provenientes de la Red de Alta precisión del IGN.

Los datos gravimétricos terrestres utilizados son procesados de acuerdo a las últimas normas para la reducción de los datos de gravedad.

En la zona marina se integran anomalías de aire libre derivadas de la altimetría satelital del modelo DNSC08 para tener un mejor conocimiento e interpretación del campo de gravedad terrestre en términos de estructuras geológicas.

Los modelos geopotenciales brindan información del campo gravitacional terrestre global y pueden representarse como una descomposición espectral en longitudes de onda que permite analizar anomalías de masa a diferentes profundidades. Mediante el filtrado de la ondulación del geoide se elimina la componente de larga longitud de onda.

A partir de la ondulación del geoide residual se identifican el sistema de Los Andes al Oeste, el sistema de Tandil y de la Ventana al Este. Se analiza a priori el estado isostático de las estructuras, mediante su correlación con las anomalías gravimétricas.

Asimismo se especula sobre las características corticales de estructuras geológicas en equilibrio isostático a partir de la interpretación de ondulaciones del geoide respecto del elipsoide de referencia. Esta metodología se aplica en el sistema de Tandil corroborando que la estructura esta en equilibrio. Los resultados obtenidos con este nuevo enfoque complementan y/o validan aquellos obtenidos a partir de análisis isostáticos antes mencionado. 


\section{Índice de Contenidos}

\section{Capítulo I Introducción}

1.1 Generalidades 1

1.2 Motivación 2

1.3 Objetivos 3

1.3.1 Objetivo general 3

1.3.2 Objetivos específicos 3

1.4 Estructura de la Tesis 4

\section{Capítulo II Anomalías de aire libre y de Bouguer. Metodología}

2.1 Estandarización de los datos gravimétricos 6

2.2 Fórmula de gravedad normal de Somigliana 7

2.3 Eliminación del efecto gravimétricos de la atmósfera 7

2.4 Corrección por altura $\quad 8$

2.5 Anomalía de la gravedad $\quad 9$

$\begin{array}{lll}2.6 & \text { Anomalía de gravedad de aire libre } & 10\end{array}$

$\begin{array}{lll}2.7 & \text { Anomalía de gravedad de Bouguer } & 10\end{array}$

2.7.1 Anomalía de Bouguer usando el modelo de placa plana 10

2.7.2 Anomalía de Bouguer usando el modelo de capa esférica 11

$\begin{array}{ll}\text { 2.7.3 Corrección topográfica } & 14\end{array}$

$\begin{array}{ll}\text { 2.7.4 Anomalía de Bouguer plana completa o refinada } & 15\end{array}$

2.7.5 Anomalía de Bouguer en el mar 15

$\begin{array}{lll}2.8 & \text { Anomalía de gravedad de un modelo geopotencial } & 16\end{array}$

\section{Capítulo III Área de trabajo y datos disponibles}

$\begin{array}{lll}3.1 & \text { Descripción general del área de trabajo } & 18\end{array}$

$\begin{array}{ll}3.2 \text { Datos disponibles } & 21\end{array}$

$\begin{array}{ll}\text { 3.2.1 Datos terrestres. Fuente IGN } & 21\end{array}$

3.2.2 Datos marinos. Fuente Danish Nacional Space Center (DNSC) 24

3.2.3 Modelo Digital de Elevación SRTM30 PLUS v7.0 24

3.2.4 Modelo Geopotencial EGM2008 26

$\begin{array}{ll}\text { 3.2.5 Modelo Digital de Elevación DTM2006 } & 27\end{array}$

Capítulo IV Cálculo de anomalías de aire libre y Bouguer 
4.1 Tratamiento de los Datos: Organización de los datos de gravedad en la zona de estudio

4.1.1 Eliminación del término de Honkasalo 28

4.1.2 Eliminación del efecto gravimétrico de la atmósfera 28

$\begin{array}{lll}4.2 & \text { Corrección de aire libre } & 29\end{array}$

4.3 Anomalía gravimétrica de aire libre 30

4.3.1 Integración de AAL de datos terrestres con AAL en el mar 32

4.4 Anomalía gravimétrica de Bouguer 35

4.4.1 Anomalía de la gravedad de Bouguer Plana Simple (ABPS) 35

4.4.2 Anomalía de la gravedad de Bouguer Esférica Simple (ABES) 36

4.4.3 Comparación entre ABPS y ABES 37

4.4.4 Corrección topográfica 39

4.4.5 Anomalía de la gravedad de Bouguer completa 40

4.5 Anomalías gravimétricas para la localización de estructuras 44

4.6 Mapas de anomalías gravimétricas 45

4.7 Conclusiones del capítulo 45

\section{Capítulo V Evaluación de resultados}

5.1 Evaluación del modelo digital de elevación DTM2006 en puntos discretos IGN 48

5.2 Evaluación del modelo digital de elevación SRTM30 Plus en puntos discretos IGN 50

5.3 Comparación entre DTM2006 y SRTM30 PLUS 50

5.4 Influencia de la altura en el cálculo de anomalías gravimétricas 52

5.5 Anomalía de aire libre del modelo neopotencial EGM2008 y su comparación con las determinadas con datos IGN más datos DNSC08 53

5.6 Anomalía de Bouguer del modelo geopotencial EGM2008 y su comparación con las determinadas con datos IGN 55

5.7 Conclusiones del capítulo 56

\section{Capítulo VI Isostasia y equilibrio isostático}

6.1 Desarrollo histórico 58

6.2 Base matemática de la hipótesis de Airy 59

6.3 Base matemática de la hipótesis de Pratt 61

$\begin{array}{lll}6.4 & \text { Equilibrio isostático } & 61\end{array}$

6.5 Determinación del equilibrio isostático 62

6.6 Análisis cualitativo de los mapas de anomalías de aire libre y anomalías de Bouguer 
6.7 Análisis de anomalías de aire libre y Bouguer en relación con la topografía sobre perfiles

6.8 Conclusiones del capítulo

\section{Capítulo VII Ondulación del Geoide del modelo neopotencial EGM2008}

$\begin{array}{lll}7.1 & \text { Ondulación del geoide } & 72\end{array}$

$\begin{array}{lll}7.2 & \text { Filtrado del geoide } & 76\end{array}$

7.3 Correlación de la ondulación del geoide residual con anomalías de aire libre y Bouguer

7.4 Conclusiones del capítulo

Capítulo VIII Análisis de las características corticales de estructuras geológicas a partir de ondulaciones del geoide isostático

$\begin{array}{lll}\text { 8.1 } & \text { Introducción } & 84 \\ \text { 8.2 } & \text { Ondulación del geoide e isostasia } & 85 \\ \text { 8.3 } & \text { Análisis isostático de estructuras } & 86 \\ 8.4 & \text { Estructura de Tandil } & 87 \\ 8.5 & \text { Análisis de resultados } & 89 \\ \text { 8.6 } & \text { Conclusiones del capítulo } & 91\end{array}$

$\begin{array}{lll}\text { Capítulo IX Conclusiones } & 92\end{array}$

Bibliografía consultada y referencias citadas en el texto 95 


\section{Lista de Figuras}

$\begin{array}{llr}\text { Figura 2.1: } & \text { Anomalía de la gravedad } & 9\end{array}$

$\begin{array}{ll}\text { Figura 2.2: } \quad \text { Placa horizontal infinita de Bouguer } & 11\end{array}$

Figura 2.3: Geometría de la capa esférica en relación con la lámina horizontal $\begin{array}{ll}\text { infinita de Bouguer } & 12\end{array}$

Figura 2.4: Corrección de Bullard B y sus primeras diferencias 13

Figura 2.5: Corrección topográfica en el modelo plano 14

$\begin{array}{ll}\text { Figura 2.6: } \quad \text { Corrección de Bouguer en el mar } & 15\end{array}$

$\begin{array}{ll}\text { Figura 3.1: } & \text { Ubicación de la zona de trabajo }\end{array}$

Figura 3.2: Mapa topográfico de la zona de trabajo 20

Figura 3.3: Marco de referencia vertical argentino 22

Figura 3.4: Ubicación de los puntos IGN en la zona de estudio 23

Figura 3.5: Error vertical absoluto del $90 \%$ para América del Sur 25

Figura 4.1: Corrección por altura 30

Figura 4.2: Correlación de las anomalías gravimétricas de aire libre con la altura 31

Figura 4.3: Anomalías gravimétricas del mar provenientes del modelo DNSC08 32

Figura 4.4: Anomalías gravimétricas de aire libre. a) AAL a partir de datos IGN b) AAL del modelo integrado (datos IGN más el modelo DNSC08) 34

Figura 4.5: Anomalías gravimétricas de aire libre del modelo integrado superpuesto sobre mapa topográfico obtenido del modelo SRTM30 PLUS 34

Figura 4.6: Residuos de anomalías gravimétricas del modelo DNSC08 a partir de grilla con datos terrestres y marinos 35

Figura 4.7: Anomalías de Bouguer simple versus alturas en puntos IGN.

a) ABPS en relación con la altura. b) ABES en relación con la altura.

c) Diferencia entre ABES y ABPS en relación con la altura

Figura 4.8: Anomalías gravimétricas de Bouguer y mapa topográfico.

a) Anomalía de Bouguer plana simple. b) Anomalía de Bouguer esférica simple. c) Diferencia entre ABES-ABPS. 38

Figura 4.9: Correcciones topográficas versus alturas en puntos IGN 39

Figura 4.10: Corrección topográfica en relación con la topografía 40

Figura 4.11: Anomalía de Bouguer plana completa versus alturas en puntos IGN 41

Figura 4.12: Mapa de anomalía de Bouguer plana completa 42

Figura 4.13: Diferencias entre las diferentes anomalías de Bouguer calculadas.

a) Diferencia entre ABPC y ABPS. b) Diferencia entra ABPC y ABES 
Figura 4.14: Anomalías de Bouguer residual. a) ABPC-ABPS. b) ABPC-ABES

Figura 4.15: Mapa de anomalías de Bouguer plana completa en tierra y anomalías de aire libre en el mar

Figura 4.16: Mapa de anomalías de aire libre para la zona de estudio integrando datos terrestres IGN y anomalías DNSC08 en el mar

Figura 5.1: Diferencias entre alturas IGN y alturas del modelo digital de elevación

Figura 5.2: $\quad$ Evaluación de diferencias de alturas entre puntos IGN y modelo digital de elevación.

Figura 5.3: Evaluación de diferencias de alturas entre modelos SRTM30 PLUS y DTM2006.

Figura 5.4: Comparación entre modelos digitales de elevación. a) Modelo DTM2006.

b) modelos SRTM30 PLUS. c) Diferencia

Figura 5.5: Evaluación de diferencias de anomalías de aire libre en nodos de grilla entre el modelo integrado (IGN+DNSC) y el EGM2008

Figura 5.6: Comparación entre anomalías de aire libre. a) Modelo integrado (IGN+DNSC08). b) EGM2008. c) Diferencias

Figura 5.7: Evaluación de diferencias de anomalías de Bouguer plana simple en nodos de grilla entre modelo calculado y EGM2008

Figura 5.8: Comparación entre anomalías de Bouguer. a) Obtenidas a partir de datos IGN. b) EGM2008. c) Diferencias

Figura 6.1: $\quad$ Primera observación de la isostasia $\quad 59$

Figura 6.2: Geometría del modelo de Airy $\quad 60$

Figura 6.3: Geometría del modlo de Pratt 61

Figura 6.4: Anomalía de Bouguer a través de la margen continental pasiva 63

Figura 6.5: Anomalías de aire libre y Bouguer. Clasificación en nodos de grilla 66

Figura 6.6: Isolíneas de anomalías de aire libre y Bouguer sobre mapa topográfico $\quad 67$

Figura 6.7: Correlación entre anomalías de aire libre y Bouguer. 68

Figura 6.8: Perfiles de anomalías gravimétricas de aire libre y Bouguer, y perfiles topográficos a lo largo de paralelos

Figura7.1: Isolíneas de ondulación del geoide del modelo EGM2008 para diferentes grados en la zona de estudio

Figura 7.2: Identificación de estructuras a partir del trazado de ondulación del geoide residual EGM2008 sobre modelo digital de elevación SRTM30 PLUS 78

Figura 7.3: Isolíneas de ondulación del geoide residual EGM2008 sobre mapa de anomalías de aire libre (arriba) y anomalías de Bouguer (abajo)

Figura 8.1: Ubicación del perfil de la estructura de Tandil sobre mapa topográfico

Figura 8.2: $\quad$ Perfil topográfico del modelo DTM2006 sobre sección de la estructura 
de Tandil

Figura 8.3: Ondulación del geoide isostático sobre sección de la estructura de Tandil 88

Figura 8.4: Ondulación del geoide residual sobre sección de estructura de Tandil 89

Figura 8.5: Ondulación geoide residual e isostático sobre sección de Tandil 90

Figura 8.6: Desvíos (ondulación geoide residual menos geoide isostático) sobre sección Tandil 


\section{Lista de Tablas}

Tabla 2.1: $\quad$ Valores de la corrección Bullard B 13

Tabla 3.1: $\quad$ Resumen del rendimiento de la altura del modelo SRTM 25

Tabla 4.1: $\quad$ Resumen estadístico de los datos de gravedad en puntos IGN 28

Tabla 4.2: $\quad$ Resumen estadístico de los datos de gravedad corregidos por efectos atmosféricos en puntos IGN 29

Tabla 4.3: Resumen estadístico de la corrección por altura usando la aproximación de primer orden para un modelo de Tierra esférica y segundo orden para un modelo de Tierra elipsoidal y las diferencias entre ambas aproximaciones en puntos IGN

Tabla 4.4: $\quad$ Resumen estadístico de los datos de gravedad corregidos por altura en $\begin{array}{ll}\text { puntos IGN } & 30\end{array}$

Tabla 4.5: $\quad$ Anomalías de aire libre en puntos IGN 31

Tabla 4.6: $\quad$ Anomalías de aire libre en el mar del modelo DNSC08 32

Tabla 4.7: $\quad$ Anomalías de aire libre del modelo integrado con datos IGN y DNSC08 33

Tabla 4.8: Estadísticos de los residuos de anomalías de aire libre del modelo DNSC08 con el modelo integrado (datos terrestres y marinos) 35

Tabla 4.9: $\quad$ Anomalías de Bouguer para los puntos IGN 36

Tabla 4.10: Diferencia entre las ABPC y las ABPS y ABES 43

Tabla 5.1: Evaluación del modelo digital de elevación global en puntos discretos IGN. Diferencias entre alturas IGN y DTM2006 y SRTM30 PLUS respectivamente 48

Tabla 5.2: $\quad$ Resumen estadístico de alturas DTM2006, SRTM30 PLUS y sus diferencias 51

Tabla 5.3: Anomalías de aire libre del modelo integrado (IGN+DNSC08), del modelo EGM2008, y sus diferencias

Tabla 5.4: Anomalías de Bouguer obtenidas a partir de datos IGN, del modelo EGM2008 y sus diferencias

Tabla 7.1: $\quad$ Resumen estadístico de ondulación del geoide N del EGM2008

Tabla 7.2: $\quad$ Relación entre las longitudes de onda del modelo neopotencial y las fuentes que las originan $\quad 76$

Tabla 7.3: $\quad$ Resumen estadístico de ondulación del geoide residual del EGM2008 77

Tabla 7.4: $\quad$ Estructuras identificadas a priori a partir de la ondulación residual $\mathrm{N}_{\mathrm{R}} \quad 79$

Tabla 7.5: $\quad$ Anomalías de aire libre y Bouguer de las estructuras identificadas 79

Tabla 8.1: $\quad$ Resumen estadístico de los desvíos entre geoide isostático y el geoide residual 91 


\title{
Lista de nomenclatura y acrónimos utilizados en el texto
}

\author{
AAL Anomalía de aire libre \\ AB Anomalía de Bouguer \\ $\mathrm{AB}_{\mathrm{m}} \quad$ Anomalía de Bouguer en el mar \\ ABES Anomalía de Bouguer esférica simple \\ ABPC Anomalía de Bouguer plana completa \\ ABPS Anomalía de Bouuer plana simple \\ BACARA Base de Calibración de la República Argentina \\ BB Corrección Bullard B \\ CHAMP Challenging Minisatellite Payload \\ $\mathrm{CB}_{\mathrm{m}} \quad$ Corrección de Bouguer en el mar \\ CT Corrección Topográfica \\ DEM Digital Elevation Model \\ DNSC08 Danish National Space Center \\ DTM2006 Digital Terrain Model 2006 \\ EGM2008 Earth Gravitational Model 2008 \\ ERS European Remote Sensing \\ FFT Fast Fourier Transform \\ GEOSAT Geodetic Satellite \\ GFO Geosat Follow On \\ GOCE Gravity Field and Steady State Ocean Circulation Explorer \\ GPS Global Positioning System \\ GRACE Gravity Recovery and Climate Experiment \\ GRS80 Geodetic Reference System 1980 \\ GTOPO30 Global 30 Arc Second Elevation Data \\ IAG International Association of Geodesy \\ ICESAT Ice, Cloud, and Land Elevation Satellite \\ ICGEM International Center for Global Earth Models \\ IGM Instituto Geográfico Militar argentino \\ IGN Instituto Geográfico Nacional argentino \\ IGSN71 International Gravity Standardization Network 1971 \\ mGal miligal \\ NASA National Aeronautics and Space Administration \\ NGA National Geospatial Intelligence Agency
}


PARN Punto Altimétrico de Referencia Normal

SIO Scripps Institution of Oceanography

SRTM Shuttle Radar Topography Mission

UCGS United States Geological Survey 


\section{Capítulo I}

\section{Introducción}

\subsection{Generalidades}

El campo de gravedad terrestre nos proporciona información de las variaciones de densidad en el interior de la Tierra. Las mediciones precisas de la aceleración de la gravedad en distintos puntos de la superficie terrestre registran las variaciones anómalas de dicha aceleración y permiten inferir cambios bruscos en la densidad. Este es el fundamento del método gravimétrico, a partir del cual se determinan estructuras geológicas en subsuperficie para una gran variedad de aplicaciones. Los observables geofísicos utilizados en esta Tesis son las anomalías de la gravedad y las anomalías del geoide.

La anomalía de la gravedad es la diferencia entre la aceleración de gravedad observada, causada por las masas de la Tierra y la rotación terrestre, y la aceleración de gravedad normal generada por una distribución de masa de referencia. La aceleración de la gravedad se observa generalmente sobre la superficie terrestre y se reduce al geoide, mientras que la aceleración de la gravedad normal se calcula sobre el elipsoide de referencia.

Los valores de gravedad observados están afectados por factores espaciales y temporales. Entre lo factores temporales más importantes podemos mencionar, el drift instrumental y las mareas terrestres. Entre los factores espaciales debemos considerar la variación de la gravedad con la altura, la existencia de masas interpuestas entre la estación y la superficie de referencia, la topografia circundante a la estación y la latitud. Los valores de gravedad observados deben ser corregidos por dichos efectos para obtener las anomalías gravimétricas. De esta forma las anomalías de gravedad pueden atribuirse a distribuciones de densidad heterogéneas en el interior de la Tierra.

Existen diferentes tipos de anomalías de gravedad de acuerdo a como se corrigen los valores observados de los mencionados efectos, por ejemplo, anomalías de aire libre y anomalías de Bouguer. Las anomalías de aire libre están fuertemente correlacionadas con la topografía es por ello que suele emplearse las anomalías de Bouguer para el análisis de perturbaciones de masa a escala regional y local (Torge, 1989).

El estado isostático o de compensación de masas de las estructuras geológicas puede ser evaluado a partir del análisis de las anomalías de aire libre y Bouguer. Si una estructura 
geológica está en equilibrio isostático las anomalías de aire libre son aproximadamente iguales a cero lejos de los bordes de la estructura, mientras que las anomalías de Bouguer están correlacionadas con la imagen especular de la topografía. De esta manera los mapas gravimétricos de aire libre y de Bouguer revelan a priori si una estructura está compensada o no. Las observaciones reales reflejan que largas longitudes de onda de la gravedad (> $100 \mathrm{~km}$ ) están compensadas ya que las señales de larga longitud de onda ven estructuras más profundas sublitosféricas.

El geoide es la superficie equipotencial del campo de gravedad terrestre que mejor ajusta al nivel medio del mar para una determinada época. Desde el punto de vista geofísico, esta superficie equipotencial pone de manifiesto la distribución de las masas terrestres y sus heterogeneidades. La figura del geoide queda definida por su altura u ondulación, que es la distancia entre el geoide y el elipsoide de referencia medida a lo largo de la normal al elipsoide.

La presencia de masas anómalas perturba y ondula la superficie equipotencial del geoide. La anomalía del geoide es inversamente proporcional a la distancia a las masas anómalas y se ve afectada por variaciones laterales de densidad situadas en un amplio rango de profundidades desde el límite núcleo-manto hasta la corteza.

Es posible especular sobre las características corticales de estructuras geológicas en equilibrio isostático a partir de la interpretación de desviaciones del geoide respecto del elipsoide de referencia (Haxby y Turcotte, 1978). Los resultados obtenidos con este enfoque complementan y/o validan aquellos obtenidos a partir de análisis isostático antes mencionado.

El avance de la geodesia espacial ha contribuido al desarrollo de modelos geopotenciales de alta calidad y resolución. Estos modelos brindan información del campo gravitacional terrestre global y pueden representarse como una descomposición espectral en longitudes de onda que permite analizar anomalías de masa a diferentes profundidades. En particular es posible detectar estructuras geológicas en la corteza mediante el filtrado de la ondulación del geoide, eliminando la componente de larga longitud de onda.

\subsection{Motivación}


Con el desarrollo de las misiones espaciales gravimétricas CHAMP ${ }^{1}$, GRACE$^{2}$ y $\mathrm{GOCE}^{3}$, se ha mejorado el conocimiento del campo de gravedad global y de sus variaciones espacio-temporales. Las variaciones temporales se han monitoreado durante un lapso de tiempo de más de diez años con una resolución espacial de 200 km y con un nivel del milímetro en términos de geoide y 0,05 mGal en términos de gravedad (Rummel, 2005). Los desafíos futuros comprenden la mejora en precisión de la gravedad en todo el rango de escalas espaciales y temporales.

Entre las aplicaciones del campo de gravedad en Ciencias de la Tierra, es de interés en esta Tesis analizar las anomalías de la gravedad y las ondulaciones del geoide como una medida de la desviación de la distribución de las masas terrestres reales de la que se basa un modelo de referencia de distribución de masas, utilizando datos terrestres y datos obtenidos a partir de modelos geopotenciales.

Respecto al tratamiento de los datos gravimétricos terrestres utilizados, los mismos son procesados de acuerdo a las últimas normas para la reducción de los datos de gravedad (Hinze et al., 2005). Las mejoras en las capacidades computacionales, un mejor conocimiento de la topografía global y un mayor interés en definir con más precisión las anomalías de la gravedad, motivó la revisión de las ecuaciones involucradas en los cálculos de las reducciones gravimetricas utilizados en forma tradicional (Hinze et al., 2005).

\subsection{Objetivos}

\subsubsection{Objetivo General}

El objetivo general de la presente Tesis consiste en identificar estructuras geológicas, que a priori pueden estar equilibradas ó no isostáticamente, a partir del filtrado de modelos geopotenciales globales y del cálculo de las anomalías de aire libre y las anomalías de Bouguer en una zona central de Argentina, que comprende variada topografía, desde llanura al Este hasta montaña al Oeste. Asimismo, se infiere sobre las características corticales a partir del análisis de la ondulación del geoide isostático.

\subsubsection{Objetivos Específicos}

\footnotetext{
${ }^{1}$ CHAMP Challenging Minisatellite Payload

${ }^{2}$ Gravity Recovery and Climate Experiment

${ }^{3}$ Gravity Field and Steady State Ocean Circulation Explorer
} 
Para alcanzar el objetivo general propuesto se establecieron los siguientes objetivos específicos:

1.- Estudiar y analizar las ecuaciones que describen los distintos tipos de anomalías gravimétricas que se pretenden interpretar.

2.- Tratar el dato gravimétrico y los modelos geopotenciales más modernos (EGM2008, Pavlis et al., 2008; EIGEN-6C, Förste et al., 2011).

3.- Integrar la información gravimétrica terrestre con anomalías de gravedad de aire libre derivadas de la altimetría satelital en el mar para obtener un mejor conocimiento e interpretación del campo de gravedad terrestre en términos de estructuras geológicas.

4.- Calcular las anomalías de Bouguer simples utilizando un modelo de placa plana y de capa esférica. Calcular las anomalías de Bouguer refinadas mediante las correcciones topográficas calculadas con un $\mathrm{DEM}^{4}$.

5.- Confeccionar mapas de anomalías de aire libre y de Bouguer para la zona de estudio.

6.- Comparar las anomalías de gravedad de Bouguer con las derivadas a partir del modelo geopotencial EGM2008 ${ }^{5}$ (Pavlis et al., 2008) con el fin de evaluar la eficacia de estos datos en el cálculo de las reducciones gravimétricas.

7.- Analizar las ondulaciones geoidales del modelo geopotencial EGM2008 en toda la zona de estudio y detectar la presencia de estructuras geológicas a partir del filtrado matemático de las ondulaciones geoidales.

9.- Analizar del estado isostático a priori de las estructuras geológicas identificadas previamente.

\subsection{Estructura de la Tesis}

Con el propósito de presentar la estructura general de la Tesis, en esta Sección se realiza una breve descripción de los contenidos.

En el Capítulo II se presentan las ecuaciones básicas para calcular las anomalías de gravedad de aire libre y Bouguer en puntos discretos de gravedad observada. Para el cálculo de las reducciones gravimetricas se consideran las últimas recomendaciones de la $\mathrm{IAG}^{6}$. Se analizan las anomalías de Bouguer tanto simples como completas. Se pone énfasis en el cálculo de las correcciones topográficas.

\footnotetext{
${ }^{4}$ Digital Elevation Model

${ }^{5}$ Earth Global Model

${ }^{6}$ International Association of Geodesy
} 
En el Capítulo III se introduce el área de estudio y se describen los datos disponibles para elaborar mapas de anomalías de aire libre y de Bouguer. Se describen brevemente los datos terrestres y aquellos derivados de fuentes satelitales.

En el Capítulo IV se calculan las anomalías de aire libre y las anomalías de Bouguer para todos los puntos $\mathrm{IGN}^{7}$ ubicados en el área de estudio. Se obtiene un mapa integrando anomalías de Bouguer en tierra y anomalías de aire libre en el mar.

En el Capítulo V se presentan los resultados de la comparación entre las anomalías de de aire libre y Bouguer calculadas en el capítulo anterior con las derivadas a partir del modelo geopotencial EGM2008 y se realiza un cuidadoso análisis entre las alturas medidas en las estaciones gravimétricas y los diferentes DEMs utilizados en el desarrollo de las distintas etapas del presente trabajo.

En el Capítulo VI se analiza el estado isostático de las estructuras geológicas correlacionando las anomalías de aire libre y Bouguer con la topografía.

En el Capítulo VII se examinan las ondulaciones del geoide del modelo geopotencial EGM2008 y se realizan filtrados matemáticos de las mismas con el fin de detectar la presencia de estructuras geológicas.

En el Capítulo VIII se infiere sobre las características de la corteza de una de las estructuras en equilibrio isostatico identificadas previamente a partir del análisis de las ondulaciones del geoide isostatico.

En el Capítulo IX se enumeran las conclusiones y se especifican trabajos a futuro que pueden realizarse en base a los resultados obtenidos en el presente trabajo de Tesis.

\footnotetext{
${ }^{7}$ Instituto Geográfico Nacional
} 


\section{Capítulo II}

\section{Anomalías de aire libre y de Bouguer Metodología de cálculo}

En este capítulo se presentan las ecuaciones básicas para calcular las anomalías gravimétricas de aire libre y Bouguer tanto simples como completas, con un modelo planar ó esférico. Se pone énfasis en el cálculo de las correcciones topográficas.

\subsection{Estandarización de los datos gravimétricos}

Los valores de gravedad de la red altimétrica nacional están expresados en el sistema IGSN71 ${ }^{1}$, que constituye la Red Internacional de Estandarización de la Gravedad del año 1971. Los valores IGSN71 incluyen una corrección que elimina la parte media del efecto de marea (Honkasalo, 1964). Esta corrección se ha considerado inapropiada debido a los errores resultantes en el cálculo del geoide a partir de los valores de gravedad corregidos por este término (Heikkinen, 1979). Así, siguiendo la recomendación de la IAG (Uotila, 1980), se debe eliminar el término de Honkasalo $\Delta \mathrm{g}_{\mathrm{H}}$ de los valores de gravedad referidos a IGSN71 mediante la adición de la corrección dependiente de la latitud geodésica $\varphi$ en miligales como se indica en la ecuación (2.1):

$$
\Delta g_{H}=0.0371\left(1-3 \operatorname{sen}^{2} \varphi\right),
$$

De esta manera se obtendrán los valores de gravedad gob_cH corregidos por el término de Honkasalo como sigue:

$$
g_{\text {Obs_cH }}=g_{\text {Obs_IGSN } 71}+\Delta g_{H} \text {, }
$$

siendo gobs_IGSN71 el valor de la gravedad observado sobre la superficie terrestre referido al sistema IGSN71.

\footnotetext{
${ }^{1}$ International Gravity Standardization Network
} 
En lo sucesivo, y por simplicidad en la nomenclatura, en esta Tesis llamaremos simplemente $\mathrm{g}_{\mathrm{Obs}}$ al valor de la gravedad observado y corregido por el término de Honkasalo.

\subsection{Fórmula de Gravedad Normal de Somigliana}

La gravedad normal o teórica $\gamma$ en función de la latitud geodésica $\varphi$, se calcula con la fórmula cerrada de Somigliana (Heiskanen y Moritz, 1967):

$$
\gamma=\frac{\gamma_{e}\left(1+k \cdot \operatorname{sen}^{2} \varphi\right)}{\left(1-e^{2} \operatorname{sen}^{2} \varphi\right)^{1 / 2}},
$$

donde $\gamma_{\mathrm{e}}$ es el valor de la gravedad normal en el Ecuador; $\mathrm{k}$ es la constante de gravedad normal $\mathrm{y} \mathrm{e}^{2}$ es el cuadrado de la primera excentricidad del elipsoide de referencia.

En este trabajo utilizaremos el GRS80 ${ }^{1}$ (Moritz, 1980) cuyos parámetros y constantes de definición se observan en el Anexo 1.

\subsection{Eliminación del efecto gravimétrico de la atmósfera}

La gravedad normal calculada con la ecuación y parámetros del GRS80 contiene el efecto gravimétrico de la masas atmosféricas, al utilizarla en el cálculo de las anomalías de gravedad se debe aplicar la corrección atmosférica $\delta g_{a t m}$.

El efecto en la gravedad de la masa de la atmósfera se calcula usando la expresión analítica descripta por Ecker y Mittermayer (1969), para GRS80 con la ecuación (2.4) (Wenzel, 1985).

$$
\delta g_{a t m}=0.874-9.9 \times 10^{-5} \mathrm{H}+3.56 \times 10^{-9} \mathrm{H}^{2},
$$

donde el efecto de la gravedad se da en miligales y la altura $\mathrm{H}$ de la estación en metros.

Esta corrección se suma a la gravedad observada en la estación. La corrección atmosférica aumenta a una anomalía de la gravedad en aproximadamente $0.86 \mathrm{mGal}$ a una altura de 100 metros y aproximadamente $0.77 \mathrm{mGal}$ a $1000 \mathrm{~m}$ (Hinze et al., 2005).

\footnotetext{
${ }^{1}$ Geodetic Reference System 1980
} 


\subsection{Corrección por altura}

La gravedad varía por una serie de efectos espaciales y temporales. Dentro de los efectos espaciales sabemos que la gravedad varía con la altura. La corrección de la altura $\delta \mathrm{g}_{\mathrm{h}} \mathrm{se}$ conoce como la corrección de aire libre y tiene en cuenta la variación de la gravedad con la elevación por encima de una determinada superficie de referencia, generalmente se adopta el geoide como superficie de referencia. Esta corrección se obtiene a partir del desarrollo en serie de Taylor del gradiente vertical de la gravedad, reteniendo los dos primeros términos de la serie podemos expresar:

$$
\delta g_{h}=-\frac{\partial g}{\partial H} H+\frac{1}{2} \frac{\partial^{2} g}{\partial H^{2}} H^{2}
$$

En la reducción de aire libre el gradiente vertical de la gravedad real se desconoce y generalmente se reemplaza por el gradiente vertical de gravedad normal

$$
\delta g_{h} \approx-\frac{\partial \gamma}{\partial h} H+\frac{1}{2} \frac{\partial^{2} \gamma}{\partial h^{2}} H^{2}
$$

Tradicionalmente basta con utilizar para esta corrección, el primer orden de una aproximación de Tierra esférica dada por la fórmula:

$$
\delta g_{h}=-0.3086 H,
$$

donde $\mathrm{H}$ es la altura de la estación en metros $\mathrm{y} \delta \mathrm{g}_{\mathrm{h}}$ en miligales.

Para mejorar la precisión de esta reducción, se recomienda el uso de una fórmula hasta un desarrollo de segundo orden de un modelo de Tierra elipsoidal (Torge, 1989). La corrección de la gravedad teórica para una altura $\mathrm{H}$ sobre el nivel del mar como se expresa en la ecuación (2.8):

$$
\delta g_{h}=-\frac{2 \gamma_{e}}{a}\left[1+f+m+\left(-3 f+\frac{5}{2} m\right) \operatorname{sen}^{2} \varphi\right] H+\frac{3 \gamma_{e} H^{2}}{a^{2}}
$$

donde $\mathrm{H}$ es la altura de la gravedad observada en $\mathrm{P}$ sobre el geoide, $\mathrm{f}$ es el aplanamiento geométrico, $\mathrm{m}$ es el parámetro geodésico, relación entre las aceleraciones gravitacional y centrifuga en el ecuador y a es el semieje mayor (radio ecuatorial) del elipsoide de referencia. 
Usando los parámetros del elipsoide del GRS80 (Anexo 1) la fórmula de segundo orden que expresa la variación de la gravedad normal con la altura y con la latitud geodésica $\varphi$ es:

$$
\delta g_{h}=-\left(0.3087691-0.0004398 \operatorname{sen}^{2} \varphi\right) H+7.2125 \times 10^{-8} H^{2},
$$

donde la altura $\mathrm{H}$ está en metros y el efecto gravimétrico está expresado en miligales.

\subsection{Anomalía de la gravedad}

La anomalía de la gravedad $\Delta \mathrm{g}$ se define como la diferencia entre la gravedad observada g en P sobre el geoide o reducida al geoide y la gravedad normal (o teórica) $\gamma$ en Q sobre el elipsoide (Heiskanen y Moritz, 1967, Hofmann y Moritz, 2005), ver Figura 2.1 y ecuación (2.10):

$$
\Delta g=g_{P}-\gamma_{Q}
$$

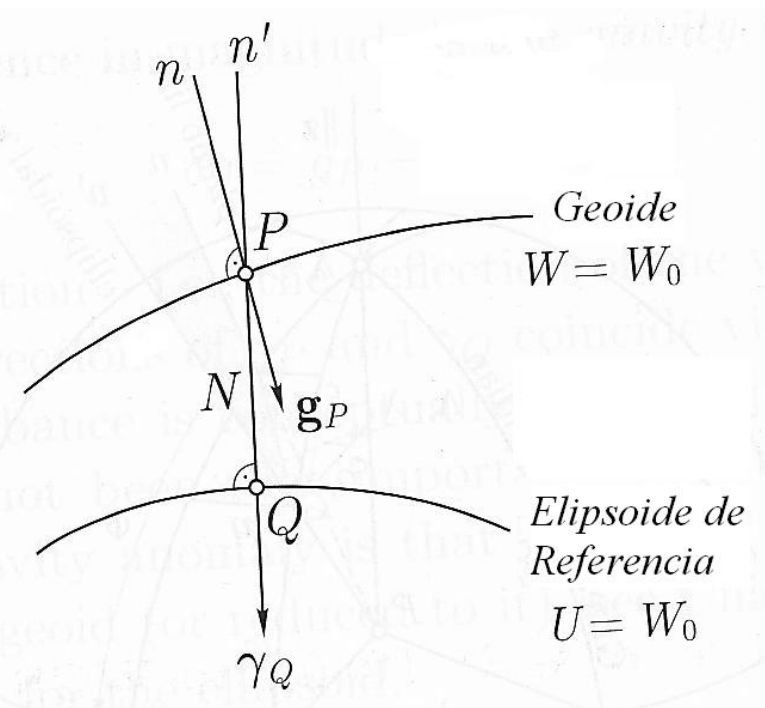

Figura 2.1 - Anomalía de la gravedad (Hofmann y Mortitz, 2005)

La distancia PQ entre el geoide y elipsoide se llama altitud del geoide u ondulación del geoide, y se designa por $\mathrm{N}$.

Generalmente, el valor de la gravedad se observa sobre la superficie terrestre y no sobre el geoide. En consecuencia, para obtener el valor de gravedad $\mathrm{g}_{\mathrm{P}}$ sobre el geoide es necesario realizar reducciones gravimétricas. 


\subsection{Anomalía de gravedad de aire libre}

La anomalía de aire libre (AAL) se obtiene aplicando la corrección de aire libre al valor de la gravedad observada para reducirla al geoide. La reducción o corrección de aire libre sólo considera el efecto de la diferencia de altura entre el punto de observación y el geoide sin considerar el efecto de las masas.

El cálculo de la anomalía gravimétrica de aire libre se obtiene con la ecuación (2.11)

$$
A A L=g_{\text {obs }}+\delta g_{a t m}+\delta g_{h}-\gamma
$$

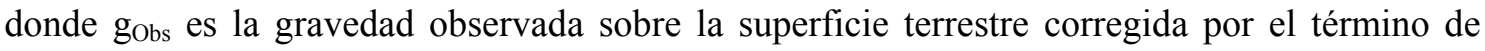
Honkasalo, $\delta g_{a t m}$ es la corrección atmosférica, $\delta g_{\mathrm{h}}$ es la corrección de aire libre y $\gamma$ es la gravedad normal sobre la superficie del elipsoide de referencia, calculada con la fórmula de Somigliana.

\subsection{Anomalía de gravedad de Bouguer}

Las anomalías de aire libre sólo consideran la variación de la gravedad con la altura y la variación por latitud pero no tienen en cuenta la masa interpuesta entre la estación y la superficie de referencia. El efecto gravimétrico de la masa topográfica contenida entre la estación y el geoide puede modelarse utilizando la atracción de una placa infinita o de una capa esférica, ambas de densidad constante.

Las anomalías de Bouguer $(\mathrm{AB})$ remueven las masas topográficas existentes entre la estación y el geoide y se calculan mediante la siguiente fórmula:

$$
A B=g_{O b s}-\delta g_{T O P}+\delta g_{a t m}+\delta g_{h}-\gamma
$$

donde, $\delta g_{\mathrm{TOP}}$ es el efecto gravimétrico de las masas topográficas.

La anomalía de Bouguer definida por la ecuación (2.12) no es exacta ya que se pueden utilizar diferentes aproximaciones sobre la forma, distribución y densidad de la masa de la topografía. Veremos dos modelos para el cálculo de esta anomalía: el modelo de lámina o placa plana y el modelo de capa esférica.

\subsubsection{Anomalías de Bouguer usando el modelo de placa plana}

La corrección de Bouguer considera el efecto gravimétrico de las masas topográfica entre la estación $\mathrm{P}$ y el geoide $\mathrm{P}_{0} \mathrm{y}$ se calcula tradicionalmente, suponiendo que la masa entre el 
datum vertical (geoide) y la estación puede ser representada por una lámina o placa horizontal infinita con espesor igual a la altura $\mathrm{H}$ de la estación (ver Figura 2.2).

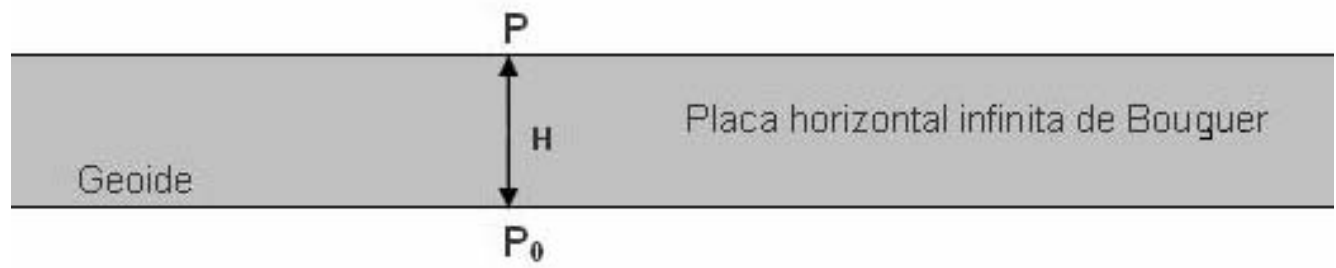

Figura 2.2 - Placa horizontal infinita de Bouguer (modificado de Hofmann y Moritz, 2005)

El efecto gravimétrico $\delta g_{\mathrm{PB}}$ para el modelo de placa plana se calcula con la ecuación (2.13) (Heiskanen y Moritz, 1967; Hofmann y Moritz, 2005):

$$
\delta g_{P B}=2 \pi G \rho H=4.193 \times 10^{-5} \rho H
$$

donde la constante gravitacional $\mathrm{G}$ es $6.673 \pm 0.001 \times 10^{-8} \mathrm{~cm}^{3} \mathrm{~g}^{-1} \mathrm{~s}^{-2}$ (Mohr y Taylor, 2001), $\rho$ es la densidad de la placa horizontal en gramos por centímetros cúbicos y $\mathrm{H}$ es el espesor de la placa plana horizontal en metros.

Si consideramos que la densidad estándar para la corteza $\rho$ es de $2.67 \mathrm{~g} / \mathrm{cm}^{3}$, entonces la ecuación (2.13) queda:

$$
\delta g_{P B}=2 \pi G \rho H=0.1119 H,
$$

donde $\mathrm{H}$ se expresa en metros y $\delta \mathrm{g}_{\mathrm{PB}}$ en miligales.

Finalmente, la anomalía de Bouguer se obtiene con la ecuación (2.15). A esta anomalía de gravedad la llamamos Anomalía de Bouguer Plana Simple (ABPS) y utiliza la aproximación $\delta \mathrm{g}_{\mathrm{TOP}} \approx \delta \mathrm{g}_{\mathrm{PB}}$.

$$
A B P S=g_{O b s}-\delta g_{P B}+\delta g_{a t m}+\delta g_{h}-\gamma
$$

\subsubsection{Anomalías de Bouguer usando el modelo de capa esférica}

En lugar de aproximar la topografía existente entre la estación y el geoide con una placa plana infinita se sugiere en las nuevas normas para la reducción de los datos de gravedad (Hinze et al., 2005), utilizar una capa esférica de radio de $166.7 \mathrm{~km}$ expresada por LaFehr (1991): 


$$
\delta g_{B E}=2 \pi G \rho[(1+\mu) H-\lambda R]
$$

donde $\mu$ y $\lambda$ son coeficientes adimensionales definidos por LaFehr, $\mathrm{R}$ es el radio de la Tierra, $\mathrm{H}$ es la altura de la estación, y $\rho$ es la densidad del material que se toma para la capa esférica.

La diferencia entre la placa de Bouguer y la capa esférica es la llamada corrección Bullard B (BB), que representa el efecto de la curvatura de la capa

$$
B B=2 \pi G \rho[\mu H-\lambda R]
$$

El efecto gravimétrico de la capa esférica es más pequeño que la formulación de la lámina horizontal debido al truncamiento convencional a $166.7 \mathrm{~km}$.

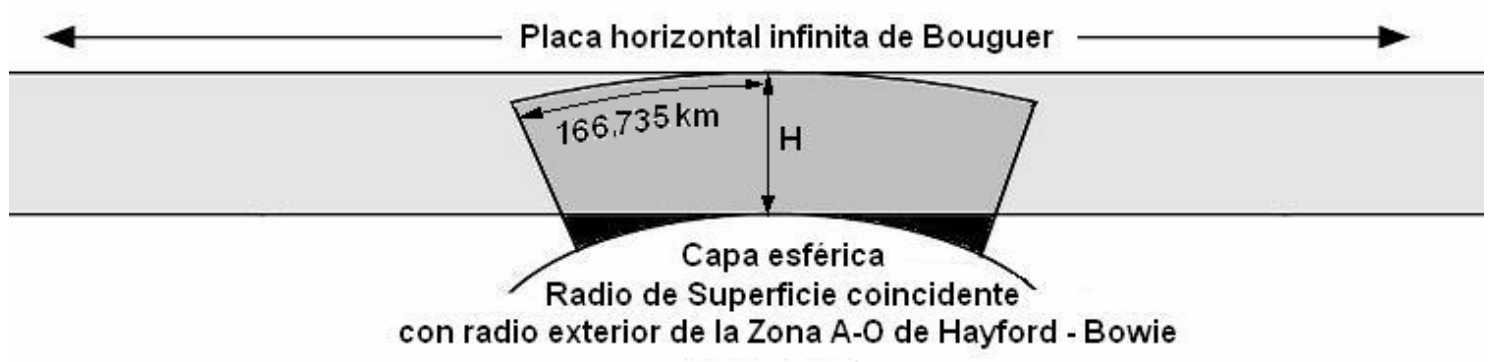

Figura 2.3 - Geometría de la capa esférica en relación con la lámina horizontal infinita de Bouguer (modificado de LaFehr, 1991)

Esta corrección fue propuesta por Bullard en 1936 y se conoce como la corrección de Bullard B o corrección esférica. El propósito de ésta corrección como paso previo al cálculo de la anomalía de Bouguer es convertir la geometría de la lámina plana infinita a una capa esférica (ver Figura 2.3) cuyo espesor es la altura de la estación y cuyo radio (longitud de arco) de la estación es 166,735 km, que es el radio exterior del sistema de terreno de Hayford-Bowie (Hayford and Bowie, 1912). El mismo corresponde a uno de los sectores (o anillos) definidos en las Tablas gravimétricas de Hayford para la determinación del efecto de la topografía y la compensación isostática (Heiskanen y Moritz, 1967). El efecto de la corrección esférica es equivalente a la eliminación de toda la lámina sobre la superficie de la Tierra y más allá de 167 kilómetros ya sea por encima o por debajo de la superficie terrestre (es decir, toda la zona de color gris claro) y la adición de la parte de la capa por debajo de la lámina (es decir, la zona de color negro sólido).

Los valores de esta corrección BB se observan en la Tabla 2.1 y la variación de esta corrección se muestra en la Figura 2.4 (LaFehr, 1991). En la Figura 2.4 se advierte que para bajas alturas predomina la capa esférica directamente subyacente a la lámina infinita (zona de 
sombreado sólido negro), es decir el efecto del truncamiento es relativamente pequeño. Para una elevación cercana a los $4000 \mathrm{~m}$ la corrección es casi cero, por lo que el efecto del truncamiento se equilibra con el material de la capa en la base de la lámina infinita. La recta de diferencias mostrado en la figura mencionada indica que esta corrección puede ser tan alta como 14 microgales por metro de diferencia de altura en alturas bajas.

Tabla 2.1.- Valores de la Corrección Bullard B (LaFehr, 1991)

\begin{tabular}{|c|c|c|c|c|c|c|c|}
\hline $\begin{array}{c}\mathbf{H} \\
(\mathbf{m})\end{array}$ & $\begin{array}{c}\text { BB } \\
\text { (mGal) }\end{array}$ & $\begin{array}{c}\mathbf{H} \\
(\mathbf{m})\end{array}$ & $\begin{array}{c}\text { BB } \\
\text { (mGal) }\end{array}$ & $\begin{array}{c}\mathbf{H} \\
(\mathbf{m})\end{array}$ & $\begin{array}{c}\text { BB } \\
\text { (mGal) }\end{array}$ & $\begin{array}{c}\mathbf{H} \\
(\mathbf{m})\end{array}$ & $\begin{array}{c}\text { BB } \\
\text { (mGal) }\end{array}$ \\
\hline 0 & 0,000 & 1600 & 1,439 & 3200 & 1,071 & 4800 & -1.099 \\
\hline 100 & 0,143 & 1700 & 1,469 & 3300 & 0,988 & 4900 & -1.295 \\
\hline 200 & 0,279 & 1800 & 1,491 & 3400 & 0,898 & 5000 & -1.497 \\
\hline 300 & 0,407 & 1900 & 1,507 & 3500 & 0,801 & 5100 & -1.707 \\
\hline 400 & 0,529 & 2000 & 1,516 & 3600 & 0,697 & 5200 & -1.923 \\
\hline 500 & 0,644 & 2100 & 1,518 & 3700 & 0,586 & 5300 & -2.147 \\
\hline 600 & 0,751 & 2200 & 1,512 & 3800 & 0,468 & 5400 & -2.377 \\
\hline 700 & 0,852 & 2300 & 1,500 & 3900 & 0,343 & 5500 & -2.615 \\
\hline 800 & 0,945 & 2400 & 1,481 & 4000 & 0,211 & 5600 & -2.859 \\
\hline 900 & 1,032 & 2500 & 1,454 & 4100 & 0,072 & 5700 & -3.111 \\
\hline 1000 & 1,111 & 2600 & 1,420 & 4200 & $-0,074$ & 5800 & -3.370 \\
\hline 1100 & 1,183 & 2700 & 1,380 & 4300 & $-0,228$ & 5900 & -3.635 \\
\hline 1200 & 1,248 & 2800 & 1,332 & 4400 & $-0,388$ & 6000 & -3.908 \\
\hline 1300 & 1,307 & 2900 & 1,278 & 4500 & $-0,555$ & 6100 & -4.188 \\
\hline 1400 & 1,358 & 3000 & 1,216 & 4600 & $-0,729$ & 6200 & -4.475 \\
\hline 1500 & 1,402 & 3100 & 1,147 & 4700 & $-0,911$ & 6300 & -4.768 \\
\hline
\end{tabular}

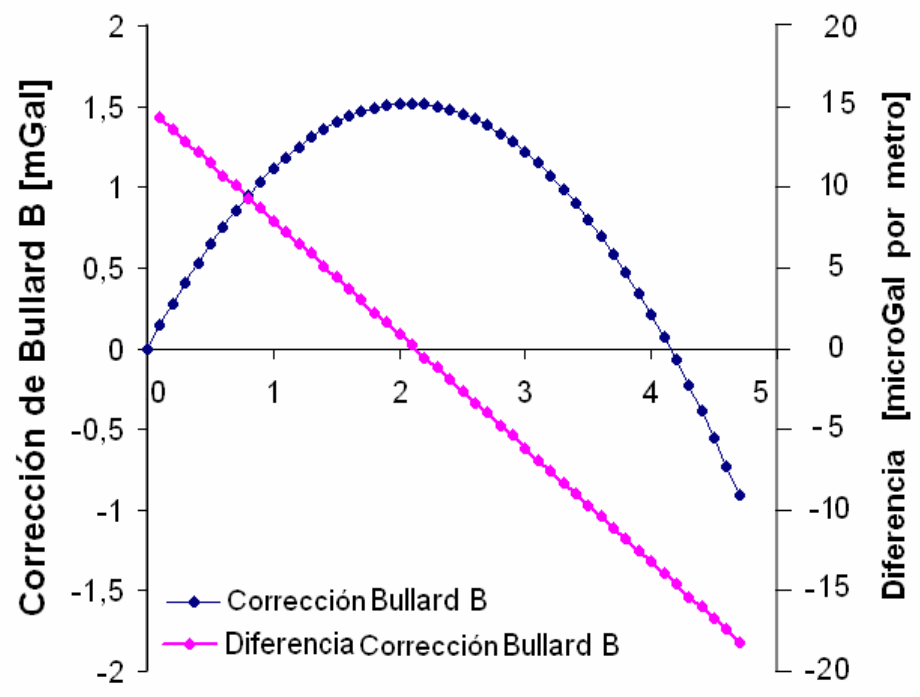

Altura $[\mathrm{km}]$

Figura 2.4 - Corrección de Bullard B y sus primeras diferencias (LaFehr, 1991)

La fórmula exacta (LaFehr, 1991) para el cálculo de los coeficientes $\mu$ y $\lambda$ de la ecuación (2.16 y 2.17) se detallan en el Anexo 1. 
La anomalía de gravedad de Bouguer esférica simple (ABES) utiliza la aproximación $\delta \mathrm{g}_{\text {TOP }} \approx \delta \mathrm{g}_{\mathrm{BE}}$, que es el efecto gravitacional de la cáscara o capa esférica de Bouguer de espesor constante $\mathrm{H}$.

$$
A B E S=g_{O b s}-\delta g_{B E}+\delta g_{a t m}+\delta g_{h}-\gamma
$$

\subsubsection{Corrección topográfica}

Tanto en el cálculo de las ABPS (ecuación 2.15) como en las ABES (ecuación 2.18) usamos modelos simples para calcular el efecto gravitacional de la masa topográfica existente entre la estación y la superficie de referencia, asemejándola a una placa horizontal infinita o a una capa esférica, ambas de espesor y densidad constante. En ambos casos no se tuvo en cuenta el exceso o defecto de masa en las cercanías de la estación.

A èstas anomalías de Bouguer llamadas simples se les suele sumar un término que considere el efecto gravimétrico del terreno residual, llamado corrección topográfica, obteniendo así las anomalías de Bouguer completas o refinadas.

La corrección topográfica se puede calcular de diferentes formas. En el modelo plano se tiene en cuenta la topografía circundante al punto de cálculo, y se suele considerar hasta una extensión de 166,735 km (zona O del sistema de Hayford-Bowie). Para este cálculo se pueden aplicar técnicas de la Transformada Rápida de Fourier (FFT), para lo cual es necesario contar con un Modelo Digital de Elevación en la región de cálculo.

La corrección topográfica plana $\delta \mathrm{g}_{\mathrm{CTP}}$ es siempre positiva para todas las estaciones en Tierra (ver Figura 2.5). En esta Tesis para el cálculo de $\delta g_{\text {СтP }}$ se utilizó el programa Tc2DFT desarrollado por Yecai Li (Li, 1993), en el Departamento de Geomática de la Universidad de Calgary (Canadá) que usa la transformada de Fourier 2D.

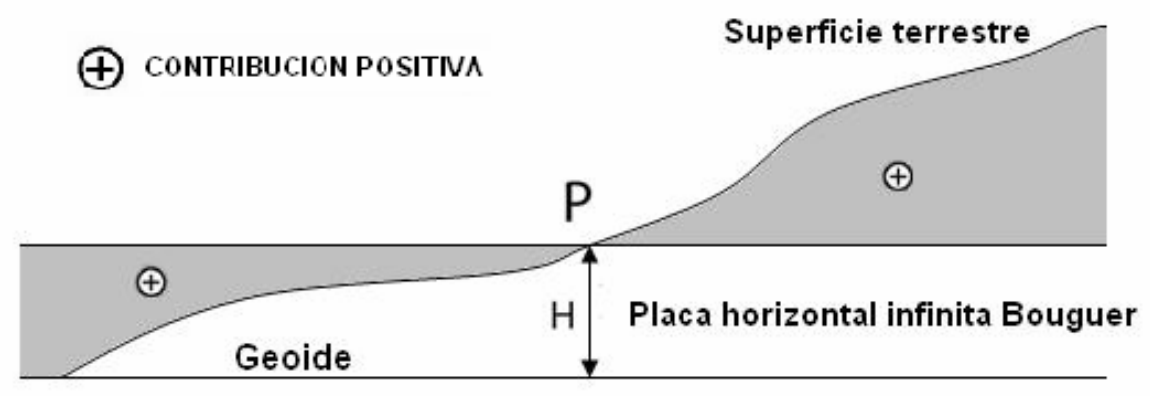

Figura 2.5 - Corrección topográfica en el modelo plano 


\subsubsection{Anomalías de Bouguer plana completa o refinada}

La corrección topográfica plana $\delta \mathrm{g}_{\mathrm{CTP}}$ se añade a la anomalía de Bouguer plana simple para dar la anomalía de gravedad de Bouguer plana completa (ABPC):

$$
A B P C=A B P S+\delta g_{C T P}
$$

De manera análoga se obtiene la anomalía de gravedad de Bouguer esférica completa (ABEC) si se añade a la anomalía de Bouguer esférica simple la corrección topográfica esférica $\delta g_{\text {CTE. }}$

\subsubsection{Anomalías de Bouguer en el mar}

En las áreas cubiertas por el mar, la gravedad, generalmente, se mide en la superficie del océano (Figura 2.7) (Lillie, 1999).

Es posible determinar la corrección de Bouguer en el mar debido a que la densidad y la profundidad del agua son bien conocidas. En lugar de remover las masas topográficas por encima del geoide, como se hace en la tierra, la corrección de Bouguer puede verse como una forma de homogeneizar a la corteza.

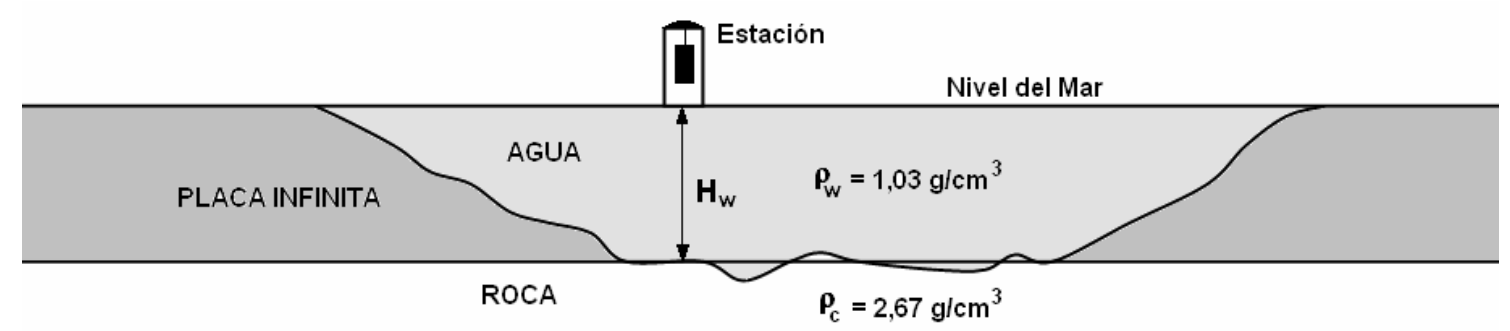

Figura 2.6 - Corrección de Bouguer en el mar (modificado de Lillie, 1999)

La corrección de Bouguer en el mar se calcula con la expresión de una placa infinita, igual a la profundidad del agua y con un contraste densidad igual a la diferencia entre la densidad del agua y la densidad de la corteza

$$
C B_{m}=0.0419\left(\rho_{w}-\rho_{c}\right) H_{w},
$$

donde $H_{w}$ es la profundidad del océano, $\rho_{\mathrm{w}}$ es la densidad del agua de mar, $\rho_{\mathrm{c}}$ la densidad de la corteza, cuyos valores se asumen $1.03 \mathrm{~g} / \mathrm{cm}^{3}$ y $2.67 \mathrm{~g} / \mathrm{cm}^{3}$, respectivamente.

$$
C B_{m}=0.0419\left(-1.64 \mathrm{~g} / \mathrm{cm}^{3}\right) H_{w}=-0.0687(\mathrm{mGal} / \mathrm{m}) H_{w},
$$


donde $\mathrm{CB}_{\mathrm{m}}$ está en miligales y la profundidad del océano en metros.

Manteniendo la convención definida anteriormente, la corrección de Bouguer en el mar $\left(\mathrm{CB}_{\mathrm{m}}\right)$, se resta a la anomalía de aire libre (AAL) para obtener las anomalías de gravedad Bouguer en el mar $\left(\mathrm{AB}_{\mathrm{m}}\right)$ :

$$
\begin{gathered}
A B_{m}=A A L-C B_{m}, \\
A B_{m}=A A L+(0.0687 \mathrm{mGal} / \mathrm{m}) H_{w} .
\end{gathered}
$$

\subsection{Anomalías de gravedad de un modelo geopotencial}

Un modelo geopotencial queda definido por el conjunto de coeficientes armónicos esféricos normalizados y adimensionales $\bar{C}_{n m}$ y $\bar{S}_{n m}$ que son determinados observacionalmente. A partir de un modelo geopotencial, cualquier magnitud del potencial terrestre puede ser calculada (Del Cogliano, 2006).

El potencial perturbador $\mathrm{T}$ definido como la diferencia entre el potencial de la gravedad real y el potencial de la gravedad normal (Heiskanen y Moritz, 1967; Hofmann y Moritz, 2005), puede expresarse en el desarrollo en serie de armónicos esféricos (Torge, 2001):

$$
T(r, \vartheta, \lambda)=\frac{G M}{r} \sum_{n=2}^{\infty}\left(\frac{a}{r}\right)^{n} \sum_{m=0}^{n}\left(\Delta \overline{C_{n m}} \cos \cdot m \lambda+\Delta \bar{S}_{n m} \cdot \operatorname{sen} \cdot m \lambda\right) \bar{P}_{n m}(\cos \vartheta)
$$

Siendo $\mathrm{n}$ el grado y $\mathrm{m}$ el orden del desarrollo en serie. Se denota con $\mathrm{r}$ la distancia geocéntrica, G la constante gravitacional, $\mathrm{M}$ la masa de la Tierra más la atmósfera, a el semieje mayor del elipsoide de referencia, $\bar{P}_{n m}$ las funciones asociadas de Legendre normalizadas, $\Delta \bar{C}_{n m}$ y $\Delta \bar{S}_{n m}$ los coeficientes armónicos esféricos, $\vartheta$ la co-latitud geocéntrica y $\lambda$ la longitud geocéntrica.

En la primera sumatoria se suele considerar el desarrollo a partir de grado $\mathrm{n}$ igual a dos, asumiendo que el origen del sistema de coordenadas coincide con el geocentro.

Los desarrollos en armónicos esféricos representan una descomposición espectral del campo gravitacional. La longitud de onda $\kappa$ asociada con el desarrollo al grado y orden $n$ (Del Cogliano, 2006) es:

$$
\kappa=\frac{360}{n} 111 \mathrm{~km} .
$$


que corresponde a una resolución de 180\% . Es decir que para el grado de 360, la longitud de onda asociada es de $111 \mathrm{~km}$, con una resolución de aproximadamente $55 \mathrm{~km}$. Para el grado 2190 la longitud de onda asociada es $18 \mathrm{~km}$ con una resolución de aproximadamente $9 \mathrm{~km}$, es decir 5 minutos de arco.

Aplicando la aproximación esférica de la ecuación de la geodesia física (ecuación 2.26) que relaciona las observaciones gravimétricas con el potencial perturbador, se obtiene la anomalía de la gravedad $\Delta \mathrm{g}$ (ecuación 2.27)

$$
\begin{gathered}
\Delta g(r, \vartheta, \lambda)=-\frac{\delta T}{\delta r}-\frac{2}{r} T \\
\Delta g(r, \vartheta, \lambda)=\frac{G M}{r^{2}}\left[\sum_{n=2}^{\infty}(n-1)\left(\frac{a}{r}\right)^{n} \sum_{m=0}^{n}\left(\Delta \overline{C_{n m}} \cos \cdot m \lambda+\Delta \overline{S_{n m}} \cdot \operatorname{sen} \cdot m \lambda\right) \bar{P}_{n m}(\cos \vartheta)\right] .
\end{gathered}
$$

La anomalía de Bouguer considera la masa interpuesta entre la superficie terrestre y la superficie de referencia, con algún modelo que estime su efecto gravitacional (ya sea con un modelo de placa plana o una capa esférica, ecuaciones (2.15) y (2.18) respectivamente). De esta manera la anomalía de Bouguer se puede obtener a partir de la ecuación (2.27) menos la atracción de la placa de Bouguer ${ }^{1}$ :

$$
\Delta g_{B}(\varphi, \lambda)=\Delta g(\varphi, \lambda)-2 \pi G \rho H(\varphi, \lambda)
$$

considerando un valor de densidad constante $\rho$ para la topografía y otra para los océanos.

\footnotetext{
${ }^{1}$ Barthelmes, F. (2002) GFZ. Scientific Technical Reports STR 09/02. Definition of Functionals of the Geopotential and their calculation from Spherical Harmonics Models.
} 


\section{Capítulo III}

\section{Área de Trabajo y Datos Disponibles}

En este capítulo se describen la ubicación de la zona de estudio y los datos disponibles para realización de la Tesis. Se describen brevemente los datos terrestres y aquellos derivados de fuentes satelitales.

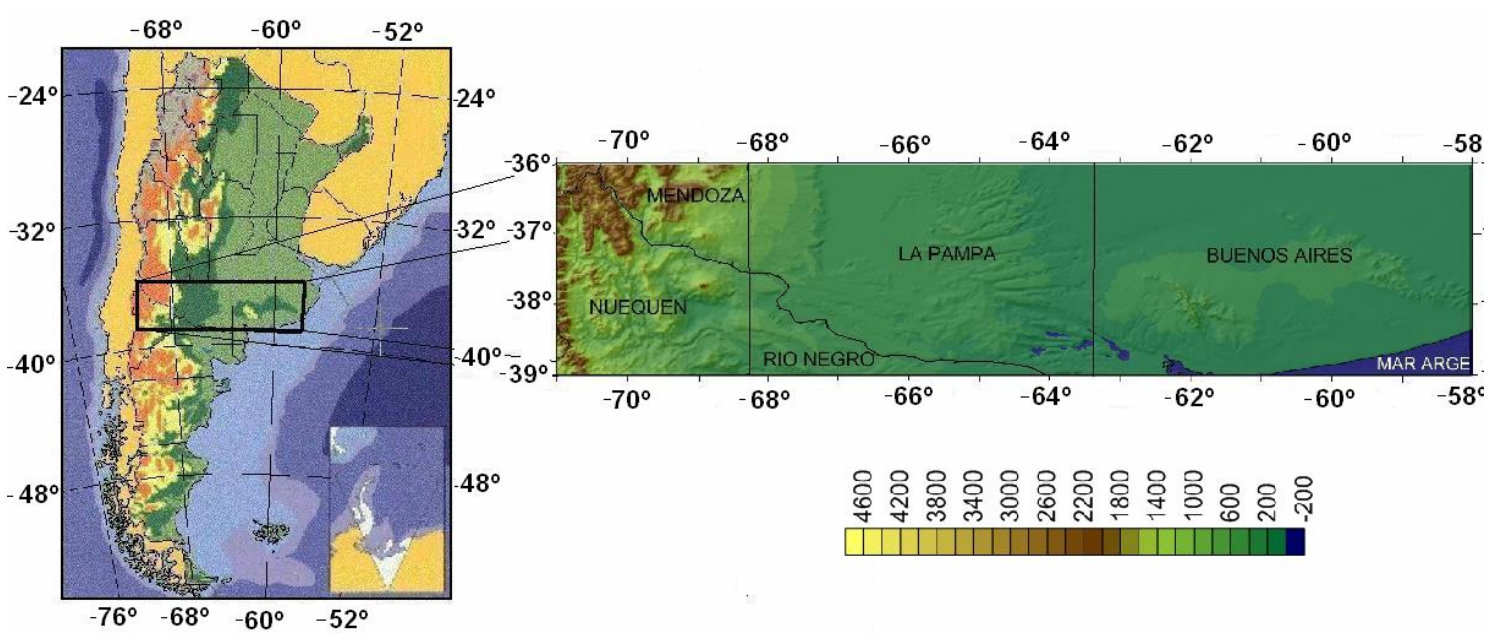

Figura 3.1 - Ubicación de la zona de trabajo. Unidad: [m]

\subsection{Descripción general del área de trabajo}

La zona de trabajo está comprendida entre las latitudes $36^{\circ} \mathrm{S}$ y $39^{\circ} \mathrm{S}$ y las longitudes $58^{\circ} \mathrm{O}$ a $71^{\circ} \mathrm{O}$. Comprende a un sector central del territorio de la República Argentina con características topográficas diferentes que incluye zona de montaña al Oeste y llanura al Este (Figura 3.1 y 3.2). Abarca gran parte de las provincias de Buenos Aires y La Pampa, y parte de las provincias de Neuquén, Mendoza y Río Negro, y cubre una pequeña fracción del mar Argentino en el extremo Sur-Este. Las alturas asumen valores entre $0 \mathrm{~m}$ en la costa al Este y superan los 4000 m en la zona de montaña al Oeste. En la Figura 3.2 se advierte que en general la zona de trabajo presenta un relieve de llanura con elevaciones bajas y de suave pendiente. Las pendientes medias en la zona de estudio son inferiores al uno por ciento en el $83 \%$ del área, y varían entre el uno y el veinte por ciento en el resto del área analizada. 
Hacia el Oeste se encuentra el sistema de Los Andes caracterizado por la presencia de cordones montañosos con alturas que llegan a los $4500 \mathrm{~m}$ y se advierte la presencia de volcanes como por ejemplo, los volcanes Domuyo (3800 m), Tromen (3300 m), y Auca Mahuida (2000 m) en la provincia de Neuquén y el volcán Payun Matrú en la provincia de Mendoza (3200 m) entre otros. La variada topografía así como la presencia de la costa marítima condicionan las variables que se analizan y justifican la elección de la zona de trabajo. 


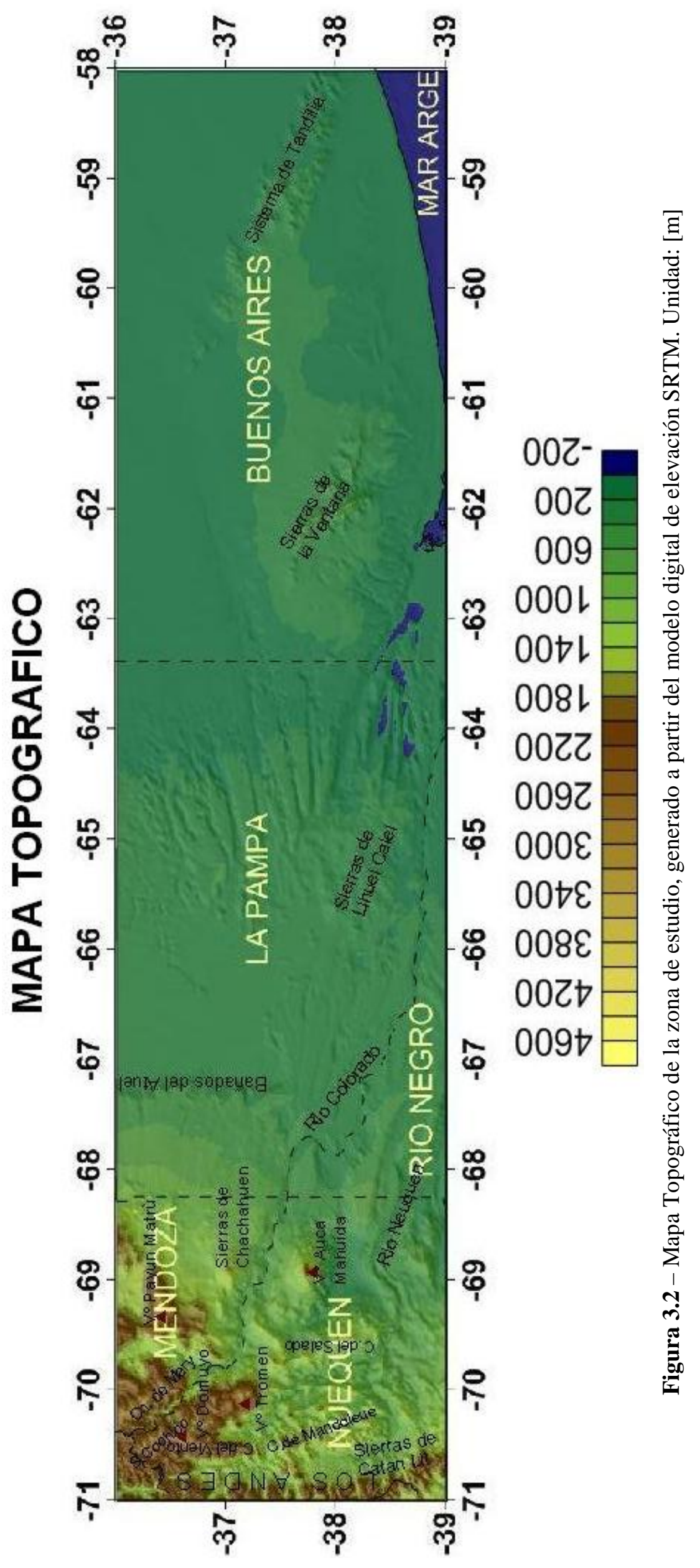




\subsection{Datos Disponibles}

\subsubsection{Datos terrestres - Fuente IGN}

El marco de referencia vertical argentino está materializado por la Red Altimétrica nacional (Figura 3.3) medida por el IGN, antes IGM ${ }^{1}$. La red altimétrica está conformada por 87.529 km de nivelación de alta precisión y por 72.805 km de nivelación topográfica y 3.250 km de nivelación auxiliar para apoyo fotogramétrico (IGM 130 IGN - 1879-2009).

Las líneas de nivelación de alta precisión dividen al territorio argentino en grandes polígonos dentro de los cuales se desarrollan las de precisión, dividiéndolos a su vez en seis u ocho polígonos menores. Las líneas de alta precisión nacen y culminan en puntos nodales, que son puntos de orden cero, y conforman polígonos o mallas. En el interior de estas mallas se desarrollan las líneas de nivelación de precisión, que están vinculadas a puntos fijos de alta precisión. Finalmente éstas se densifican con las líneas de nivelación topográfica. La separación media entre los puntos altimétricos no supera los $9 \mathrm{~km}$. Todos los puntos de la red de nivelación de alta precisión cuentan con valores de gravedad medidos.

El origen del sistema vertical argentino se definió en el año 1923, a partir del análisis de una corta serie de registros efectuados por el mareógrafo de Mar del Plata. Posteriormente, hacia 1950, su materialización física fue trasladada a las Sierras de Tandil, donde el basamento rocoso cristalino garantiza una estabilidad sísmica y geológica perdurable en el tiempo. Desde entonces el origen está materializado por el denominado Punto Altimétrico de Referencia Normal (PARN) de Tandil (Brunini et al., 2001).

La Red Gravimétrica nacional articula subredes de diferentes órdenes (ver Figura 3.3):

- Red de Orden Cero: compuesta por cinco puntos de gravedad absoluta que fueron medidos en dos etapas en los años 1989 y 1991 con el gravímetro interferométrico JILAG 3 de la Universidad de Hannover (Alemania). Estos puntos son: Miguelete y Tandil (Provincia de Buenos Aires), San Lorenzo (Salta), Comodoro Rivadavia (Chubut) y San Juan (San Juan).

- Red de Primer Orden: compuesta por 86 puntos localizados en los aeródromos que integran la denominada red BACARA (Base de Calibración de la República Argentina). Esta red fue medida en 1986.

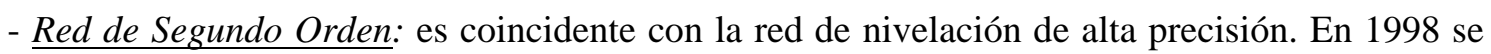
completó la medición gravimétrica de los 15.905 puntos que integran la citada red.

- Red de Tercer Orden: está constituida por parte de la red de nivelación topográfica. Hasta el presente se midieron 2.175 puntos.

El total de los puntos gravimétricos medidos por el IGM hasta el año 2001 es de 18.248 (IGM 130 IGN - 1879-2009). En 1945 se adopta como punto Datum gravimétrico provisorio, al

\footnotetext{
${ }^{1}$ Instituto Geográfico Militar
} 
pilar del Observatorio Astronómico de la Universidad Nacional de La Plata. En 1952 se comienza con las observaciones con gravímetros relativos y el establecimiento del pilar, donde se constituiría la Estación Fundamental Buenos Aires “A”. La llamada Operación BACARA (Base de Calibración de la República Argentina) constituiría lo que fue la primera Red Gravimétrica de Orden Superior de nuestro país. Pocos años después se mide la Red IGSN71 con desvíos del orden de la décima de mGal. Esta red toma 11 puntos de la Red BACARA.
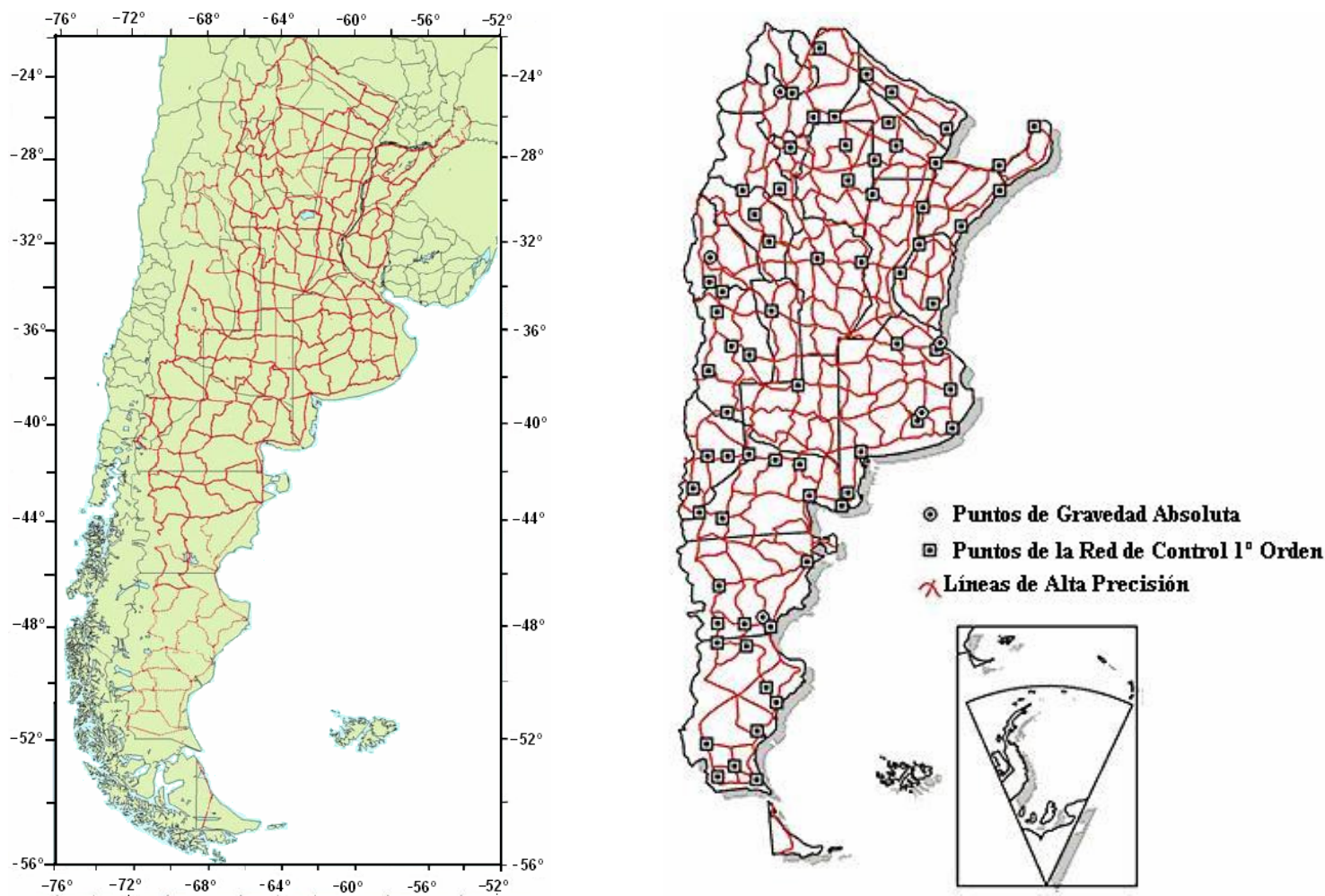

Figura 3.3 - Marco de referencia vertical argentino. Red de Nivelación (izquierda) y Red Gravimétrica (derecha). (Fuente: IGN)

\section{Estado actual de la información gravimétrica}

Casi el 85 \% de los puntos fijos de nivelación cuenta con información gravimétrica, (Villella. y Pacino, 2010). Los datos gravimétricos disponibles fueron inicialmente referidos a la Red Potsdam y posteriormente transformados a IGSN71, mediante una corrección constante de -14.93 mGal. Luego de una serie de verificaciones hoy se puede decir que la precisión de los valores de gravedad disponibles, es en el peor de los casos, de $0.5 \mathrm{mGal}$ (Villella y Pacino, 2010). 


\section{Datos usados para el área de trabajo}

En la zona de estudio se dispone de 2695 puntos de la Red de Nivelación de alta precisión del IGN con valores de posición, cota y gravedad observada. Los puntos se encuentran distribuidos en toda la zona de estudio siguiendo itinerarios de nivelación con puntos espaciados a distancias variables entre 3 a $10 \mathrm{~km}$. (Figura 3.4).

La posición de los puntos está definida en el sistema WGS84 con valores de latitud y longitud expresados en décimas de grado. La precisión de los mismos es variable.

Las alturas de los puntos de nivelación están referidas al Datum Vertical Local argentino establecido en el mareógrafo de Mar del Plata, provincia de Buenos Aires. Las alturas son alturas niveladas y están expresadas en metros.

Los puntos de nivelación se distribuyen en itinerarios que, en general, siguen las vías de comunicación como caminos y vías férreas. Las alturas de los puntos IGN en la zona de estudio, están comprendidas entre $3 \mathrm{~m}$ al Este y $2202 \mathrm{~m}$ al Oeste. Nótese que las alturas de la zona de trabajo, de acuerdo al modelo SRTM ${ }^{2}$ que describiremos a continuación llegan a superar valores de $4000 \mathrm{~m}$ al Oeste.

Los valores de gravedad corresponden al sistema IGSN71, el total de puntos de nivelación se advierten huecos de datos de gravedad, resultando sólo 2429 puntos con gravedad. Estos puntos se encuentran ubicados a la Figura 3.4 en color blanco. Los puntos rojos son puntos de la red de nivelación que contienen posición y cota pero carecen de valores de gravedad observada. Los valores de gravedad están expresados en unidades de miligal.

En este trabajo se usaron los 2429 puntos de la red de nivelación de alta precisión del IGN con posición, cota y gravedad.

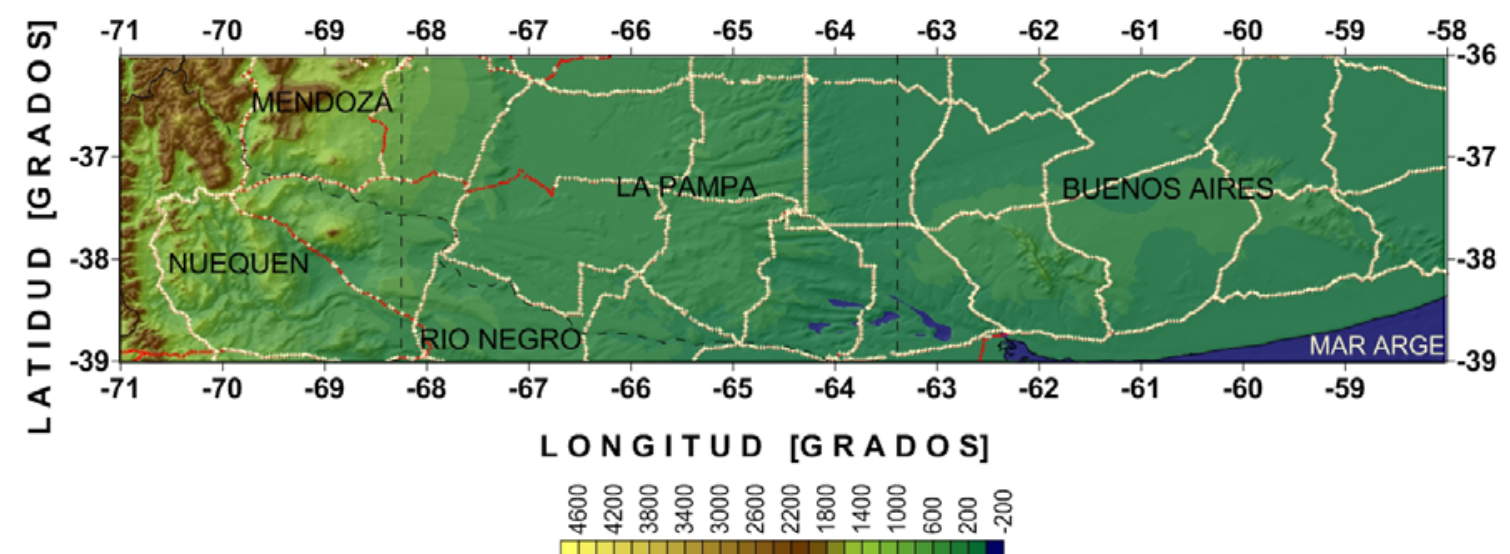

Figura 3.4 - Ubicación de los puntos IGN en la zona de estudio. Puntos blancos con gravedad y cota. Puntos rojos sin gravedad

\footnotetext{
${ }^{2}$ Shuttle Radar Topography Mission
} 


\subsubsection{Datos marinos - Fuente Danish National Space Center (DNSC)}

El DNSC08GRA es un modelo de anomalías de la gravedad derivado de la altimetría satelital proporcionados por el DNSC ${ }^{3}$. Este modelo es una actualización de DNSC07 y se basa en datos de la altimetría satelital (Andersen and Knudsen, 2008) de diversas fuentes. Los datos provienen de técnicas de rastreo (retracked) de las misiones ERS ${ }^{4}-1$ y ERS-2, datos del satélite GEOSAT $^{5}$ y GFO ${ }^{6}$ diseñados para medir la altura de la superficie marina por técnicas radar, datos de altimetría del ICESAT ${ }^{7}$ para latitudes altas, y datos de la topografía de la superficie de los océanos de las misiones TOPEX/Poseidon y JASON-1. El resultado es un modelo de anomalías gravimétricas con una resolución espacial de un minuto (aproximadamente dos kilómetros sobre la superficie terrestre). Este modelo usa el modelo geopotencial EGM2008 como modelo de referencia, (Pavlis et al., 2008).

Se usaron las anomalías gravimétricas del modelo DNSC08 en el extremo Sur-Este de la zona de trabajo correspondiente a un sector del Mar Argentino (Figuras 3.2 y 3.4). Los valores de anomalías gravimétricas están expresados en unidades de miligal.

\subsubsection{Modelo Digital de Elevación SRTM30 PLUS V7.0}

El Modelo Digital de Elevación SRTM30 PLUS (Becker et al., 2009) es un modelo global de topografía/batimetría del Scripps Institution of Oceanography (SIO) de la Universidad de San Diego, California de los Estados Unidos; de 30 segundos de arco por 30 segundos de arco ( $\sim$ km por 1 km de resolución espacial). En áreas continentales, el modelo está basado en alturas del terreno de la Misión Topográfica de Radar del Trasbordador Espacial Endeavour de la Agencia Espacial Norteamericana (NASA) (Farr et al., 2007) y en datos del DEM GTOPO30 del United States Geological Survey (USGS) en zonas de latitudes altas donde los datos SRTM no están disponibles. En regiones marinas, SRTM30 PLUS se basa en un actualizado modelo batimétrico global que combina información batimétrica inferida de datos altimétricos satelitales, con sondeos de profundidad desde barcos (Sandwell y Smith, 2001). Las coordenadas están expresadas en el sistema WGS84 y las alturas están referidas al Modelo Geopotencial EGM96.

La precisión de las elevaciones del SRTM fue analizada en detalle por varios autores (Rodríguez et al., 2005; Farr et al., 2007). Para la evaluación del modelo a nivel global se realizaron comparaciones en puntos de control terrestre, cuyas alturas fueron determinadas

\footnotetext{
${ }^{3}$ Danish Nacional Space Center

${ }^{4}$ European Remote Sensing

${ }^{5}$ Geodetic Satellite

${ }^{6}$ Geosat Follow On

${ }^{7}$ Ice, Cloud, and land Elevation Satellite
} 
independientemente usando posicionamiento GPS cinemático. Dichas comparaciones indican que el error absoluto de la elevaciones SRTM, con un nivel de confianza del 90\% (corresponde 1,5 de la desviación estándar), tienen un rango entre $\pm 6 \mathrm{~m} \mathrm{a} \pm 10 \mathrm{~m}$, dependiendo del área geográfica. El error esperado para el modelo SRTM es de $16 \mathrm{~m}$, sin embargo los resultados obtenidos de la validación muestran que la precisión vertical absoluta es mejor que 9 m, y para Sudamérica el error absoluto es de 6.2 m (Rodríguez, 2005; Farr et al., 2007), (Ver Tabla 3.1).

Tabla 3.1.- Resumen del rendimiento de la altura del modelo SRTM. Las cantidades representan los errores del 90\% en metros. (Rodríguez et al., 2005; Farr et al., 2007).

\begin{tabular}{l|cccccc}
\hline & África & Australia & Eurasia & Islas & $\begin{array}{c}\text { América } \\
\text { del Norte }\end{array}$ & $\begin{array}{c}\text { América } \\
\text { del Sur }\end{array}$ \\
\hline $\begin{array}{l}\text { Error Absoluto por } \\
\text { Geo-localización }\end{array}$ & 11.9 & 7.2 & 8.8 & 9.0 & 12.6 & 9.0 \\
\hline Error en altura absoluto & 5.6 & 6.0 & 6.2 & 8.0 & 9.0 & 6.2 \\
\hline Error en altura relativo & 9.8 & 4.7 & 8.7 & 6.2 & 7.0 & 5.5 \\
\hline $\begin{array}{l}\text { Error altura de larga longitud de } \\
\text { onda }\end{array}$ & 3.1 & 6.0 & 2.6 & 3.7 & 4.0 & 4.9 \\
\hline
\end{tabular}

En la Figura 3.5 se muestra la estructura espacial del error vertical donde se advierte que los mayores errores están asociados con pendientes empinadas como en Los Andes.
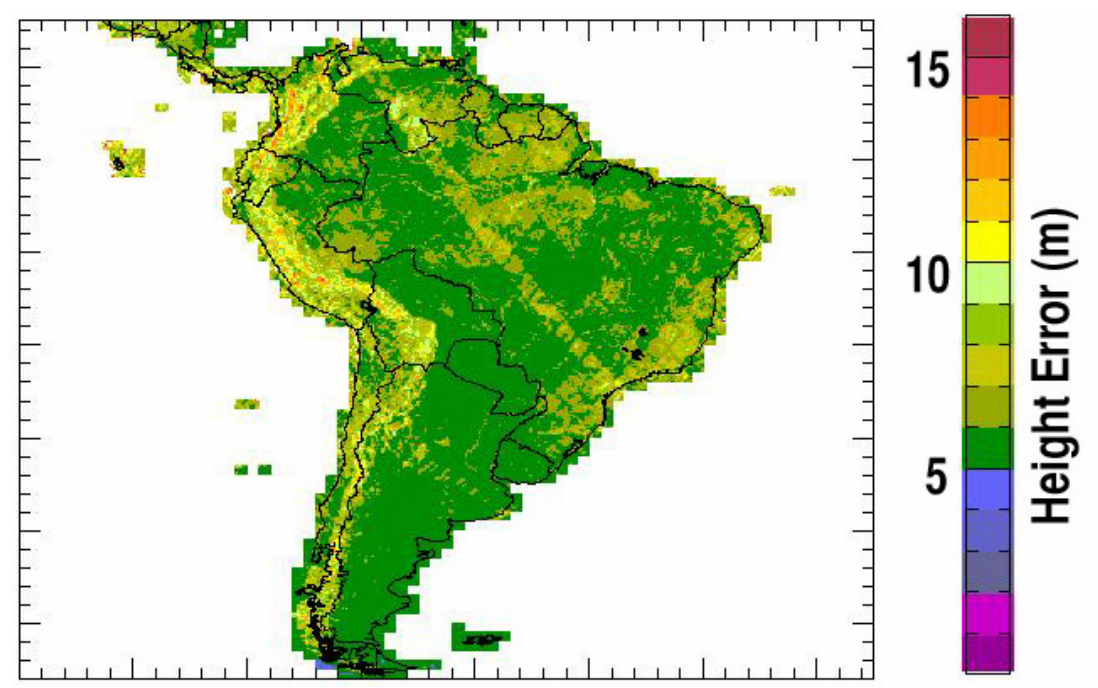

Figura 3.5 - Error vertical absoluto del 90\% para América del Sur (Rodríguez et al., 2005; Farr et al., 2007)

Se usó el modelo SRTM30 PLUS para calcular las correcciones topográficas para obtener las anomalías gravimétricas de Bouguer completas en los puntos discretos IGN. 


\subsubsection{Modelo Geopotencial EGM2008}

EGM2008 es un modelo del potencial gravitacional terrestre en términos de armónicos esféricos (Pavlis et al., 2008) de la NGA ${ }^{8}$ desarrollado por una combinación de datos de la misión GRACE (Tapley et al., 2005), los cuales contribuyen al modelo hasta el grado y orden 180 a través de la solución ITG GRACE03S (Mayer-Gür, 2008), con información contenida en una base de datos global compilada por la NGA de anomalías de gravedad de aire libre con una resolución de 5 minutos de arco. Esta base de datos contiene datos de fuentes terrestres, aerotransportadas y derivadas de la altimetría satelital. Es un modelo completo de grado y orden 2159, asimismo contiene coeficientes de armónicos esféricos adicionales que se extienden hasta grado 2190 y orden 2159, con una resolución espacial de 5 minutos de arco ( $9 \mathrm{~km}$ de resolución espacial). Es el modelo geopotencial global de mayor resolución espacial disponible hasta el presente.

El predecesor del EGM2008, fue el modelo geopotencial EGM96 (Lemoine et al., 1998) con coeficientes armónicos esféricos hasta grado y orden 360. Los mismos fueron obtenidos a partir de una solución compuesta en la que se usaron diferentes técnicas de estimación. Para los grados más bajos (hasta grado 70) la solución contiene una combinación datos de satélite, gravedad en superficie y datos de altimetría satelital, para los grados 71 a 359 se usaron anomalías de gravedad medias contenidas en una grilla con una resolución espacial de 30 minutos de arco y para el grado 360 la solución se obtuvo por métodos de cuadratura. La desventaja más significativa era la discontinuidad del error cuando se cambia la técnica de estimación (Pavlis et al., 2012).

El éxito de las misiones espaciales gravimétricas, en particular de la misión GRACE, trajo como consecuencia una mejora en la calidad de las soluciones sólo con datos de satélite ampliando la solución hasta el grado 180. Esta solución fue combinada con anomalías de gravedad medias con una resolución de 5 minutos de arco para logar una solución hasta el grado 2159 usando técnicas de estimación por mínimos cuadrados. Se desarrollaron tres modelos gravitacionales preliminares: PGM2004 (Pavlis et al., 2005), PGM2006 (Pavlis et al., 2006) y PGM2007 (Pavlis et al., 2007), que sirvieron para comprobar la calidad de la solución. La solución final del EGM2008 asimismo contiene coeficientes de armónicos esféricos adicionales que se extienden hasta grado 2190 y orden 2159.

En áreas cubiertas con alta calidad de los datos de gravedad, las discrepancias entre las ondulaciones del geoide EGM2008 y valores GPS/nivelación independientes, son del orden de $\pm 5 \mathrm{~cm}$ a $\pm 10 \mathrm{~cm}$ (Pavlis et al., 2012), mientras en Argentina las discrepancia son mayores

\footnotetext{
${ }^{8}$ National Geospatial Intelligence Agency
} 
(Pacino y Tocho, 2009). Las desviaciones de la vertical del EGM2008 comparado con valores astrogeodésicos independientes en Estados Unidos y Australia se encuentran dentro de $\pm 1,1 \mathrm{a} \pm$ a 1.3 segundos de arco. Estos resultados indican que EGM2008 resulta comparable con modelos detallados de geoide regional contemporáneos. EGM2008 representa una mejora con respecto al modelo EGM96, predecesor del EGM2008, en un factor de seis en resolución, y por factores de tres a seis en precisión.

\subsubsection{Modelo Digital de Elevación DTM2006}

DTM2006.0 (Pavlis et al; 2006) es el modelo digital de elevación que usa el calculador del ICGEM $^{9}$ para obtener las alturas del geoide a partir de las anomalías de altura y las anomalías de Bouguer en términos de armónicos esféricos. El calculador del ICGEM ofrece la posibilidad de obtener de manera separada la topografía con este modelo. La resolución del mismo es la del EGM2008 (Pavlis et al., 2008), es decir 5 minutos de arco. Las coordenadas están expresadas en el sistema WGS84 y las alturas están referidas al EGM2008.

El DTM2006.0 cuenta con una base de datos compilada por la NGA que se obtuvo por la fusión de datos SRTM y datos del DTM2002 (Saleh y Pavlis, 2003) en tierra, datos de elevaciones de hielo derivados del altímetro láser ICESAT y datos del proyecto BEDMAP, del British Antartic Survey (BAS) sobre Groenlandia y Antártida, respectivamente. Sobre el océano, el DTM2006.0 contiene esencialmente la misma información que el DTM2002, que se origina en estimaciones de valores de batimetría, altimetría y sondeos de profundidad de (Smith y Sandwell, 1997). El DTM2006.0 fue compilado en 30 segundos de arco por 30 segundos de arco de resolución (que proporciona la altura e información de profundidad solamente), en 2 minutos de arco por 2 minutos de arco y 5 minutos de arco por 5 minutos de arco de resolución, donde las profundidades de lagos y los datos de espesor de hielo también están incluidos.

\footnotetext{
${ }^{9}$ International Center for Global Earth Models
} 


\section{Capítulo IV}

\section{Cálculo de anomalías de aire libre y Bouguer}

En este capítulo se calculan las anomalías de gravedad de aire libre y de Bouguer para todos los puntos IGN ubicados en el área de estudio. Se determinan tanto las anomalías de gravedad de Bouguer simples como las completas. Para la posterior detección de estructuras geológicas se obtiene un mapa de anomalías de Bouguer en tierra y anomalías de aire libre en el mar.

\subsection{Tratamiento de los Datos: Organización de los datos de gravedad en la zona de estudio}

\subsubsection{Eliminación del término de Honkasalo}

La base de datos gravimétricos está homogenizada en el sistema IGSN71, siguiendo las recomendaciones de la IAG (Uotila, 1980) se ha eliminado el término de Honkasalo (Heikkinen, 1979) con las ecuaciones (2.1) y (2.2). Esta corrección es función de la latitud, y tiene un valor muy pequeño, cuya magnitud promedio para la gravedad observada en la zona de estudio es $0.004 \mathrm{mGal}$. El resumen estadístico de los datos de gravedad originales y los que resultaron luego de la corrección se muestran en la Tabla 4.1.

Tabla 4.1.- Resumen estadístico de los datos de gravedad en puntos IGN

\begin{tabular}{l|c|c}
\hline & Gravedad observada & $\begin{array}{c}\text { Gravedad corregida } \\
\text { término Honkasalo }\end{array}$ \\
\hline Número Puntos: & 2429 & 2429 \\
\hline Valor Máximo de gravedad [mGal]: & 980069.45 & 980069.44 \\
\hline Valor Mínimo de gravedad [mGal] : & 979228.75 & 979228.75 \\
\hline Rango de valores de gravedad [mGal] : & 840.70 & 840.69 \\
\hline Promedio gravedad [mGal]: & 979861.59 & 979861.58 \\
\hline Desviación estándar gravedad [mGal]: & \pm 125.83 & \pm 125.83 \\
\hline
\end{tabular}

\subsubsection{Eliminación del efecto gravimétrico de la atmósfera}

La corrección del efecto gravimétrico de la masa atmosférica (ecuación 2.4) es necesaria ya que una de las constantes fundamentales (GM) que define al GRS80 incluye la masa de la atmósfera. 
Para la zona de estudio esta corrección toma valores entre 0.873 y 0.673 mGal para alturas comprendidas entre 4 y 2200 m de elevación, respectivamente. La corrección se aplica a los valores de gravedad observados y corregidos por el término de Honkasalo. El resumen estadístico se muestra en la Tabla 4.2.

Tabla 4.2.- Resumen estadístico de los datos de gravedad corregidos por efectos atmosféricos en puntos IGN

\begin{tabular}{l|r}
\hline & \multicolumn{1}{|c}{ Gravedad corregida efectos atmosféricos } \\
\hline Número Puntos: & 2429 \\
\hline Valor Máximo de gravedad [mGal]: & 980070.32 \\
\hline Valor Mínimo de gravedad [mGal] : & 979229.42 \\
\hline Rango de valores de gravedad [mGal] : & 840.89 \\
\hline Promedio gravedad [mGal]: & 979862.43 \\
\hline Desviación estándar gravedad[mGal]: & \pm 125.85 \\
\hline
\end{tabular}

\subsection{Corrección de aire libre}

La corrección por altura, también conocida como corrección de aire libre, es función de la latitud y de la altura de la estación. De acuerdo a las recomendaciones de las nuevas normas para la reducción de la gravedad se aplica esta corrección a los valores de gravedad de los puntos IGN usando el gradiente vertical de segundo orden de la gravedad de un modelo de Tierra elipsoidal (ecuación 2.9) en lugar de la aproximación de primer orden para una Tierra esférica usada tradicionalmente (ecuación 2.7).

Las alturas de los puntos IGN en la zona de trabajo varían entre $4 \mathrm{~m}$ a $2200 \mathrm{~m}$ sobre el nivel del mar. La diferencia entre aplicar la corrección con una u otra aproximación es, en promedio, de $0.063 \mathrm{mGal}$, llegando a una diferencia máxima de $0.69 \mathrm{mGal}$ para la altura de $2200 \mathrm{~m}$. El detalle estadístico de los valores obtenidos para ésta corrección usando ambas aproximaciones y sus diferencias se muestra en la Tabla 4.3.

Tabla 4.3.- Resumen estadístico de la corrección por altura usando la aproximación de primer orden para un modelo de Tierra esférica y segundo orden para un modelo de Tierra elipsoidal y las diferencias entre ambas aproximaciones en puntos IGN

\begin{tabular}{l|r|r|r}
\hline & $\begin{array}{c}\text { (1) Aprox. } 1^{\circ} \text { orden } \\
\text { para Tierra esférica }\end{array}$ & $\begin{array}{c}\text { (2) Aprox. } 2^{\circ} \text { orden } \\
\text { para Tierra elipsoidal }\end{array}$ & Diferencia \\
\hline Número Puntos: & 2429 & 2429 & 2429 \\
\hline Máximo [mGal]: & -1.47 & -1.47 & 0.69 \\
\hline Mínimo [mGal] : & -679.87 & -679.18 & 0.00 \\
\hline Rango [mGal] : & 678.40 & 677.71 & 0.69 \\
\hline Promedio [mGal]: & -92.84 & -92.77 & 0.06 \\
\hline Desviación estándar [mGal]: & \pm 99.62 & \pm 99.53 & \pm 0.08 \\
\hline
\end{tabular}


En la Figura 4.1 se grafican las diferencias en función de la variación de altura donde se advierte que para bajas elevaciones ambas aproximaciones son consistentes entre sí hasta los $500 \mathrm{~m}$ de altura, con diferencias menores a $0.1 \mathrm{mGal}$. Sin embargo para mayores elevaciones la diferencia aumenta, tomando valores hasta $0.69 \mathrm{mGal}$ par alturas de $2200 \mathrm{~m}$.

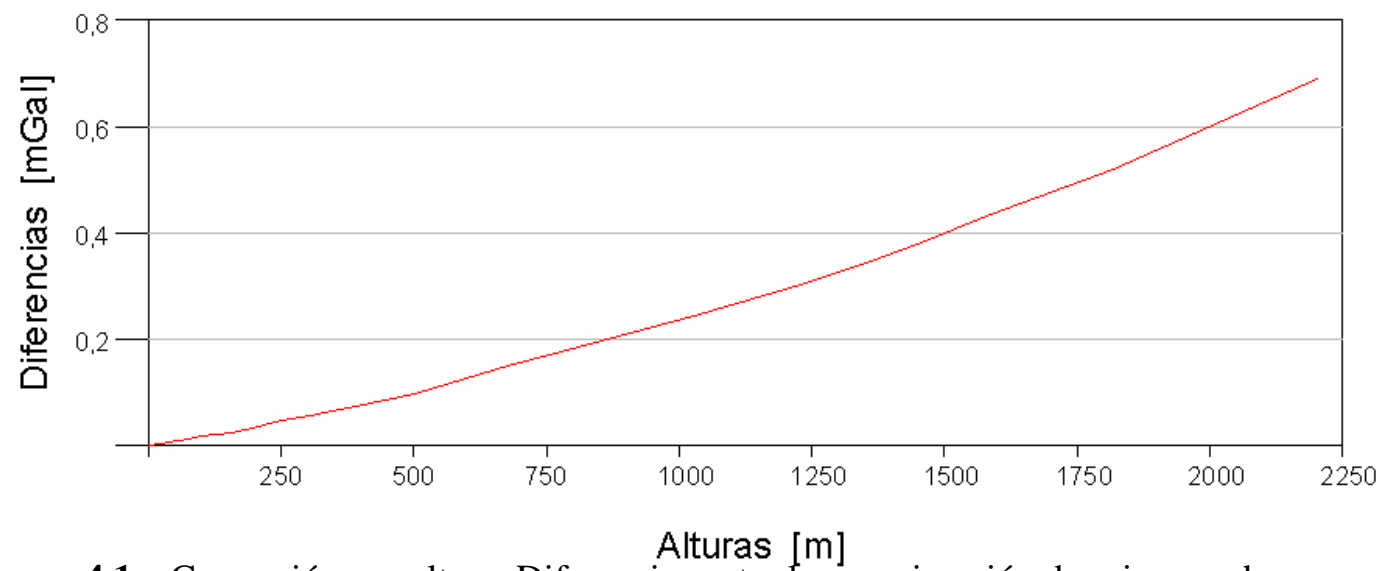

Figura 4.1 - Corrección por altura. Diferencias entre la aproximación de primer orden para una Tierra esférica y la de segundo orden para una Tierra elipsoidal, en función de la altura

De acuerdo a los resultados obtenidos y siguiendo las recomendaciones de las nuevas normas para la reducción de los datos de gravedad (Hinze et al., 2005) para el conjunto de puntos de nivelación IGN se usó el gradiente vertical de segundo orden de la gravedad, para un modelo de Tierra elipsoidal, según la ecuación (2.9). Estas correcciones se sumaron a los valores de gravedad corregidos por el término de Honkasalo (ecuación 2.1) y de los efectos atmosféricos (ecuación 2.4), obteniendo de esta manera valores de gravedad correspondientes al nivel del geoide. El resumen estadístico se muestra en la Tabla 4.4.

Tabla 4.4.- Resumen estadístico de los datos de gravedad corregidos por altura en puntos IGN

\begin{tabular}{l|r}
\hline & \multicolumn{1}{|c}{ Gravedad corregida efectos atmosféricos } \\
\hline Número Puntos: & 2429 \\
\hline Valor Máximo de gravedad [mGal]: & 980067.53 \\
\hline Valor Mínimo de gravedad [mGal] : & 978550.24 \\
\hline Rango de valores de gravedad [mGal] : & 1517.29 \\
\hline Promedio gravedad [mGal]: & 979769.65 \\
\hline Desviación estándar gravedad [mGal]: & \pm 214.46 \\
\hline
\end{tabular}

\subsection{Anomalía gravimétrica de aire libre}

Se calculan las anomalías gravimétricas de aire libre (AAL) en la región de estudio, utilizando el gradiente de segundo orden vertical de la gravedad, efectos gravimétricos de la 

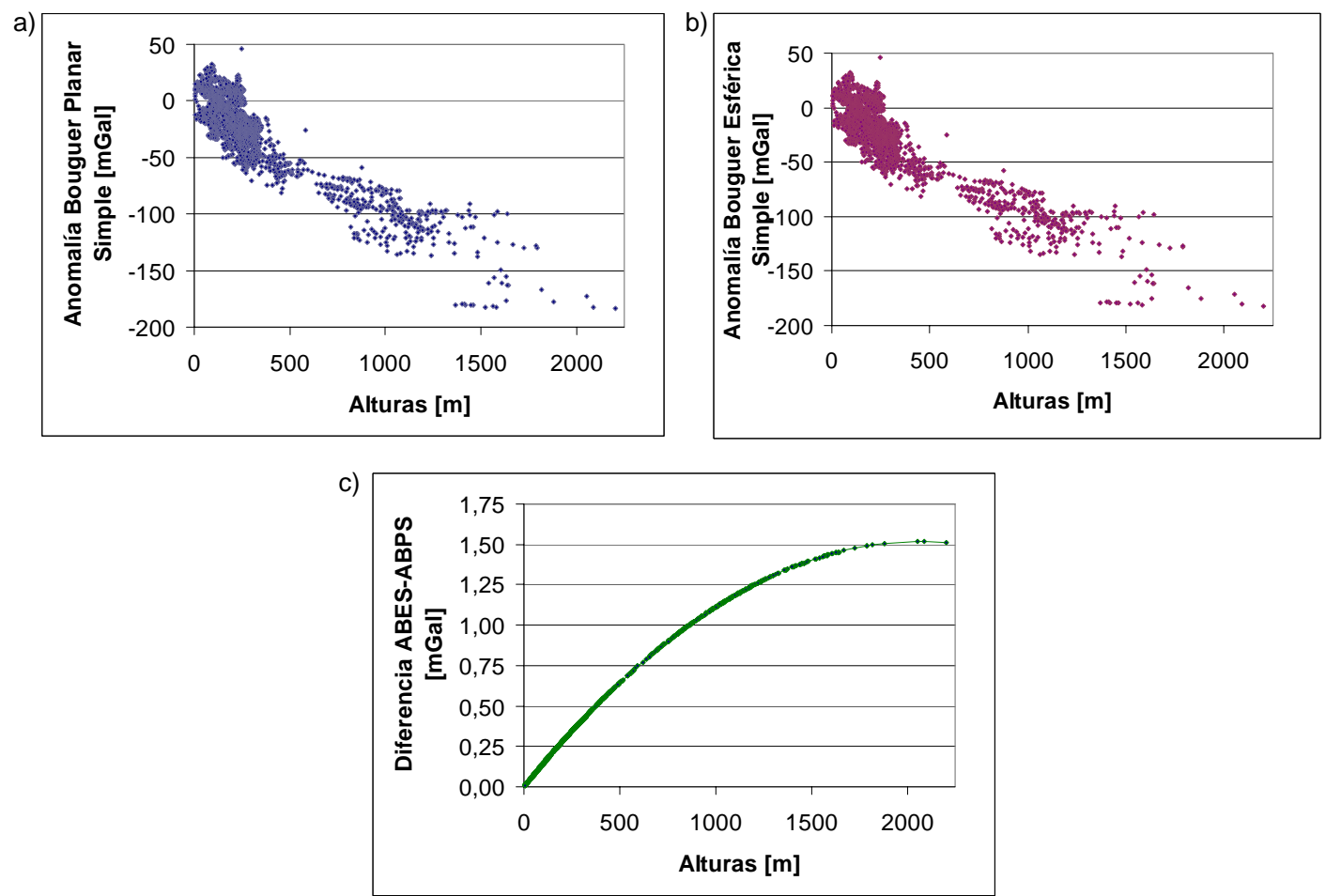

Figura 4.7 - Anomalías de Bouguer simple versus alturas en puntos IGN. a) ABPS en relación con la altura. b) ABES en relación con la altura. c) Diferencia entre ABES y ABPS en relación con la altura

\subsubsection{Comparación entre ABPS y ABES}

Siendo muy similares los valores obtenidos de ABES y ABPS se realizó la diferencia entre ambos, obteniéndose los valores que se muestran en la Figura 4.7.c para el caso de los puntos discretos. Las diferencias son siempre positivas, es decir que la atracción de la capa esférica es superior a la placa plana de Bouguer. Las diferencias toman valores entre 0.007 mGal para las menores alturas y llegan a $1.50 \mathrm{mGal}$ para las más altas, lo cual muestra que el efecto del truncamiento de la capa esférica es insignificante para bajas elevaciones, y alcanza valores de $1.50 \mathrm{mGal}$ para alturas de $2200 \mathrm{~m}$. La curva representada por los puntos de la Figura 4.7.c se corresponde con la Figura 2.4 que muestra la corrección de Bullard B.

Para visualizar la estructura espacial de las discrepancias entre las ABES y las ABPS, se generó una grilla haciendo la diferencia entre grillas de las ABES menos las ABPS (Figura 4.8.c). En la misma se advierte que si bien el comportamiento espacial de las ABES y ABPS parece idéntica, las magnitudes no lo son, y las diferencias entre ambas se corresponden con el hecho de que las ABES son superiores a las ABPS. Este comportamiento ya lo hemos advertido en los puntos discretos IGN (Figura 4.7.c). Los valores siempre positivos de la grilla con diferencias confirman la tendencia mostrada en el análisis en los puntos discretos, sobre el mayor efecto gravitacional de la capa esférica sobre el efecto de la placa de Bouguer, al menos para alturas hasta los 4500 metros. 


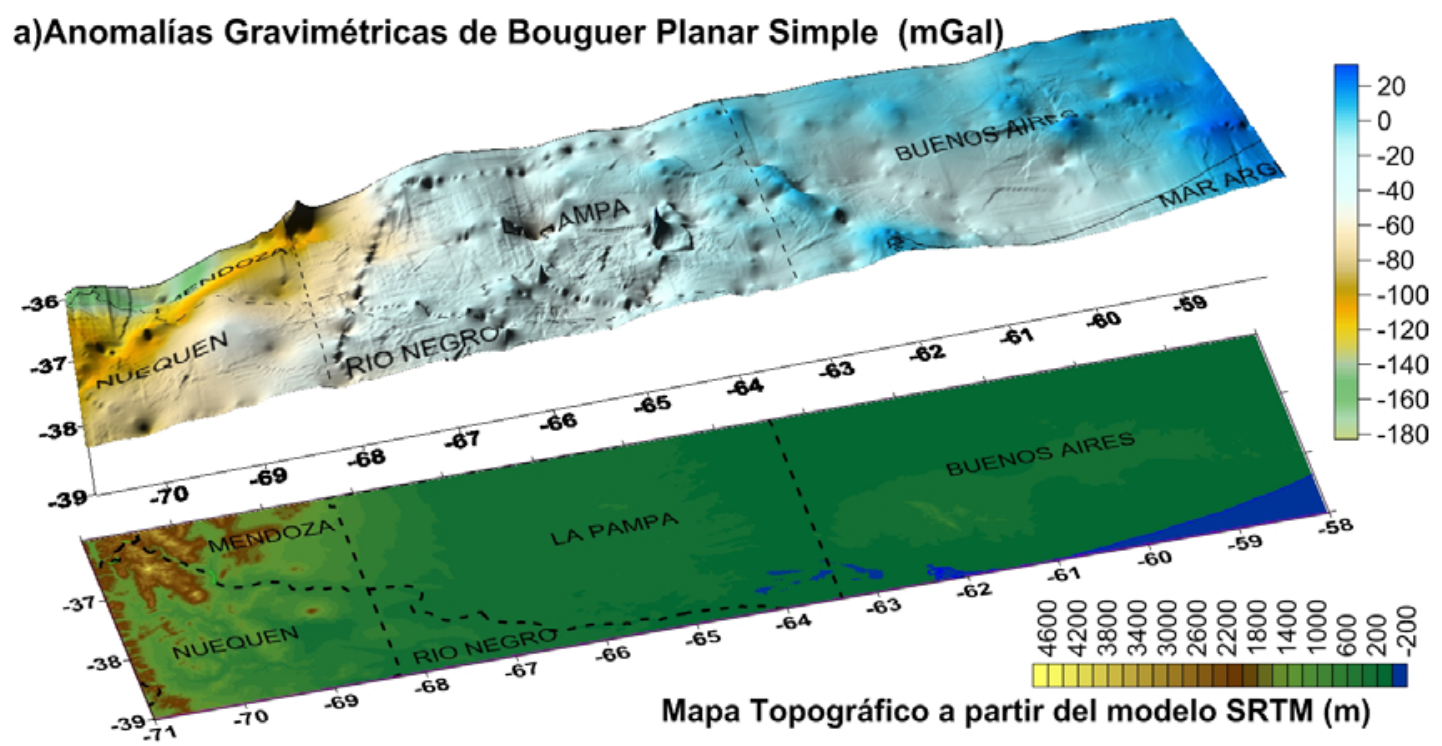

b)Anomalías Gravimétricas de Bouguer Esférica Simple (mGal)
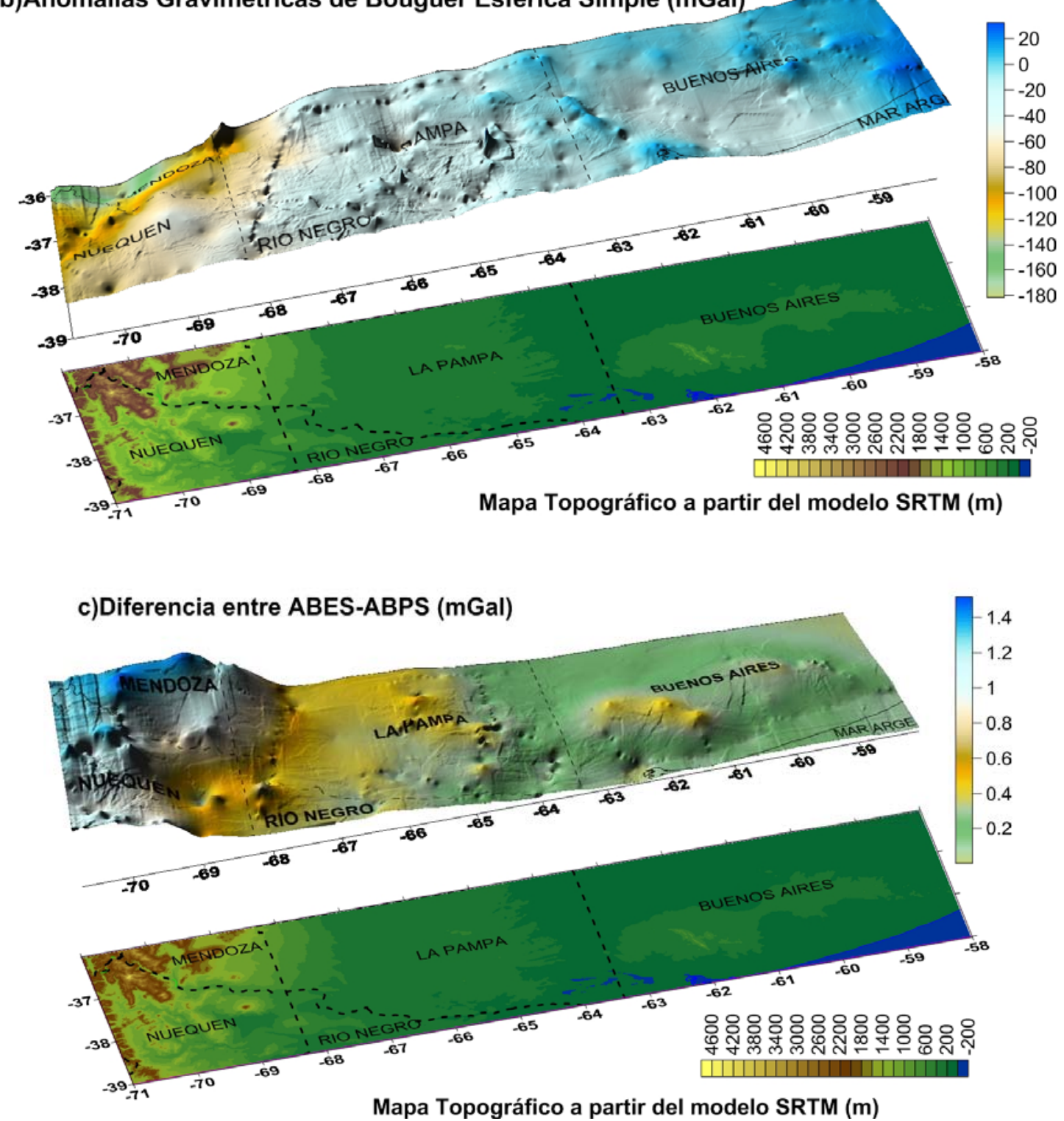

Figura 4.8 - Anomalías gravimétricas de Bouguer y mapa topográfico. a) Anomalía de Bouguer plana simple. b) Anomalía de Bouguer esférica simple. C) Diferencia entre ABESABPS. Las anomalías están expresadas en mGal y las alturas en metros 
En las sierras de Tandil al Este, las alturas llegan a valores de $600 \mathrm{~m}$, se advierte que las diferencias entre el modelo esférico y plano toman valores cercanos a $0.6 \mathrm{mGal}$, esto se corresponde con la Figura 2.4 que muestra que el efecto de la corrección de Bullard B en función de la altura. Se advierte un comportamiento similar hacia el Oeste, en el sistema de Los Andes, donde para alturas aproximadamente de 2000 metros la diferencia de las anomalías de Bouguer entre ambos modelos llega a 1.5 mGal (LaFehr, 1991; Hinze et al., 2005).

\subsubsection{Corrección Topográfica}

El efecto gravitacional de las masas topográficas circundantes al punto de cálculo, ya sea en exceso o en defecto, no considerado por la placa de Bouguer es tenido en cuenta mediante la corrección topográfica.

Para el cálculo de la corrección topográfica se usó el programa Tc2DFT (Li, 1993) que usa la transformada de Fourier 2D utilizando una aproximación planar.

La corrección topográfica CT obtenida con Tc2DFT (Li, 1993) toma valores siempre positivos que se suman a las anomalías de Bouguer planas simples para obtener las anomalías de Bouguer completas o refinadas. En la Tabla 4.9 se muestran los estadísticos (máximo, mínimo, promedio y desviación estándar) de estas correcciones.

A fin de analizar la correlación de las CT con la altura se muestra en la Figura 4.9, que los valores CT, siempre positivos, tienden a crecer con la altura, esto es más claro para alturas superiores a $500 \mathrm{~m}$.

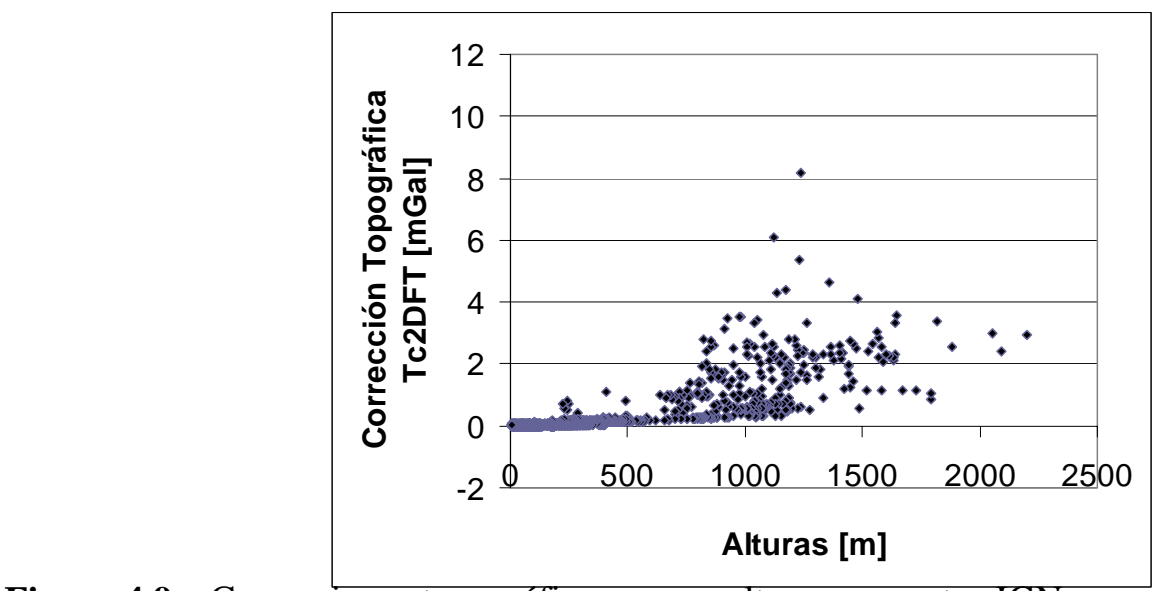

Figura 4.9 - Correcciones topográficas versus alturas en puntos IGN

Para analizar la estructura espacial de las CT, se generó una grilla para la zona de estudio (Figura 4.10). En la misma se advierte que para el 90\% de la zona los valores de las CT 
son inferiores a $1 \mathrm{mGal}$, en tanto que un $5 \%$ toma valores de 1 a $2 \mathrm{mGal}$ y el restante $5 \%$ toma valores entre los 2 a $8 \mathrm{mGal}$. Claramente los valores superiores a $1 \mathrm{mGal}$ se corresponden con el sistema de Los Andes.

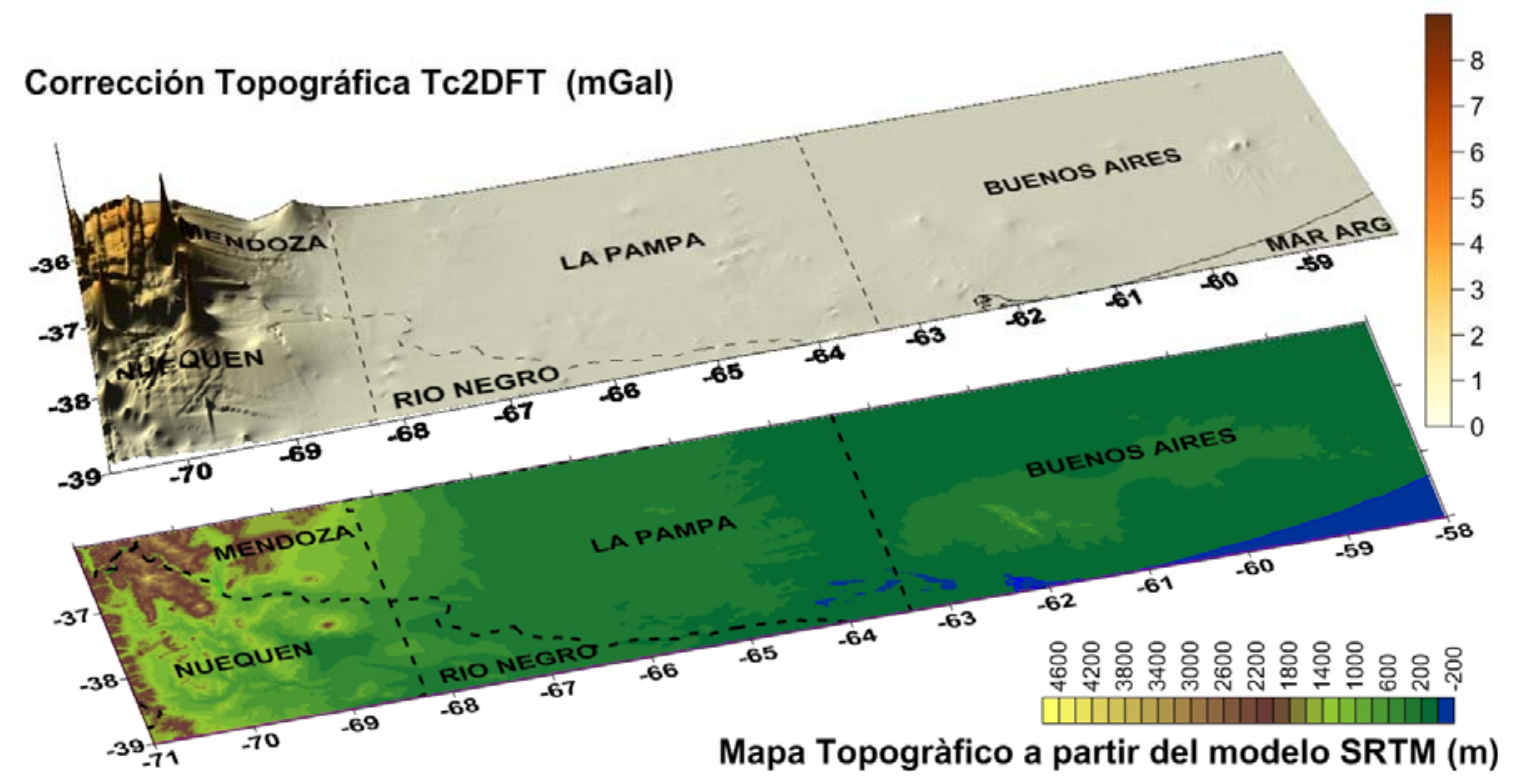

Figura 4.10 - Corrección topográfica en relación con la topografía

\subsubsection{Anomalía de la Gravedad de Bouguer Completa}

Se calcula la anomalía de Bouguer plana completa (ABPC) con la ecuación (2.19), sumando a la anomalía de Bouguer plana simple (ABPS) (ecuación 2.15) la corrección topográfica (CT).

Para la corrección topográfica se usó el modelo SRTM30 Plus V.7.0 (Becker et al., 2009), con una resolución espacial de 30 segundos de arco ( $1 \mathrm{~km}$ en el Ecuador). En el cálculo de la CT se consideró la topografía en las inmediaciones del punto de cálculo (hasta los 166.735 km). El resumen estadístico de los valores CT se resumen en la Tabla 4.9.

Los valores de las ABPC en los puntos IGN obtenidas con la corrección CT varían entre -180.95 mGal a $45.90 \mathrm{mGal}$. Los valores negativos de menor magnitud que las ABPS se deben a las magnitudes positivas de la CT. En la Figura 4.11 se muestra la correlación negativa de las ABPC con las alturas, esto es más evidente para alturas superiores a los 500 metros. Para analizar el comportamiento espacial de las ABPC, se generó una grilla con resolución de un minuto ( $1 \mathrm{~km}$ en el Ecuador) y como método de interpolación se usó Kriging lineal (Figura 4.12); en la misma se aprecia que se mantiene la correlación negativa de las anomalías de Bouguer con las alturas. 


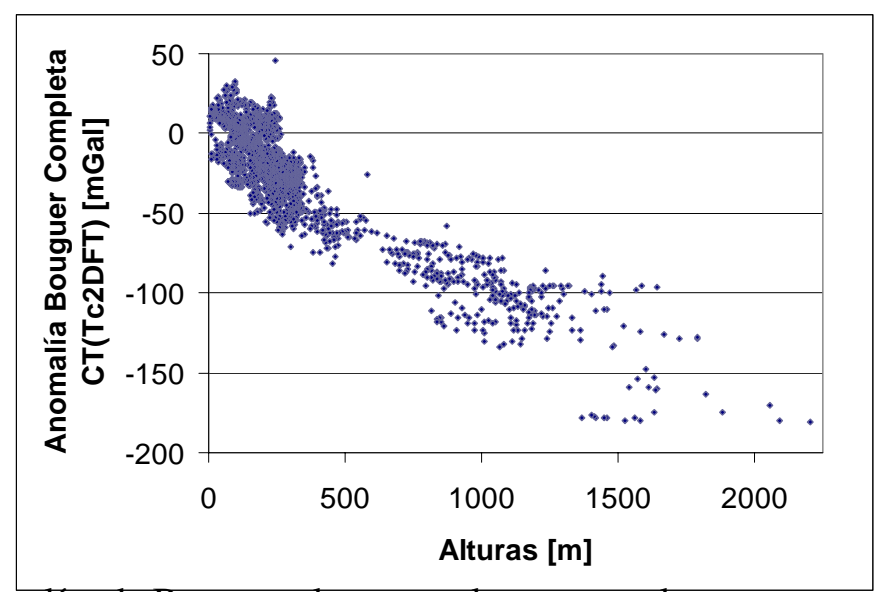

Figura 4.11 - Anomalías de Bouguer plana completa versus alturas en puntos IGN

Los valores resumidos en la Tabla 4.9 y el mapa mostrado en la Figura 4.12., muestran anomalías de la gravedad de Bouguer muy negativas (alrededor de -180 mGal hacia el Oeste). Esta es una característica muy conocida de las anomalías de Bouguer en tierra, lo que indica que las masas topográficas están generalmente compensadas isostáticamente por anomalías de masa en la litosfera, por lo menos para largas longitudes de onda (mayor a cientos de kilómetros) (Watts, 2001, Kuhn et al, 2009) 
masa de la atmósfera y la fórmula de gravedad normal de Somigliana para el Sistema de Referencia Geodésico de 1980 según las ecuaciones (2.7), (2.4) y (2.3), respectivamente.

El cálculo de la AAL se obtuvo con la ecuación (2.9). El resumen estadístico de los AAL obtenidos para los 2429 puntos IGN se muestra en la Tabla 4.5.

Tabla 4.5.-Anomalías de aire libre en puntos IGN

\begin{tabular}{l|r}
\hline Num. Puntos & 2429 \\
\hline Valor Máximo de AAL [mGal] & 83.92 \\
\hline Valor Mínimo de AAL[mGal] & -37.24 \\
\hline Promedio [mGal] & 7.60 \\
\hline Desviación estándar [mGal] & \pm 16.17 \\
\hline
\end{tabular}

Las AAL están correlacionadas positivamente con la altura del punto donde se midió la gravedad debido al efecto gravitacional de las masas topográficas en la corta longitud de onda (Uotila, 1967; Torge, 1989).

Con el fin de analizar la correlación de las AAL con las alturas H de los puntos IGN se realizó la gráfica de dispersión que se muestra de la Figura 4.2. Si hubiera una dependencia funcional exacta entre las anomalías gravimétricas de aire libre y la altura, todos los puntos estarían sobre una recta (o, con más generalidad, sobre una curva). En realidad, sólo hay una relación funcional aproximada, una tendencia general de las anomalías de aire libre a crecer linealmente con la altura; excepciones, incluso grandes, son posibles (Hofmann y Moritz, 2005). En la Figura 4.2 se advierte una falta de correlación de las AAL con las alturas evidenciado por una dispersión aleatoria que se manifiesta hasta alturas de $500 \mathrm{~m}$. A partir de $500 \mathrm{~m}$ se observa una leve correlación de las AAL positiva en función de las alturas.

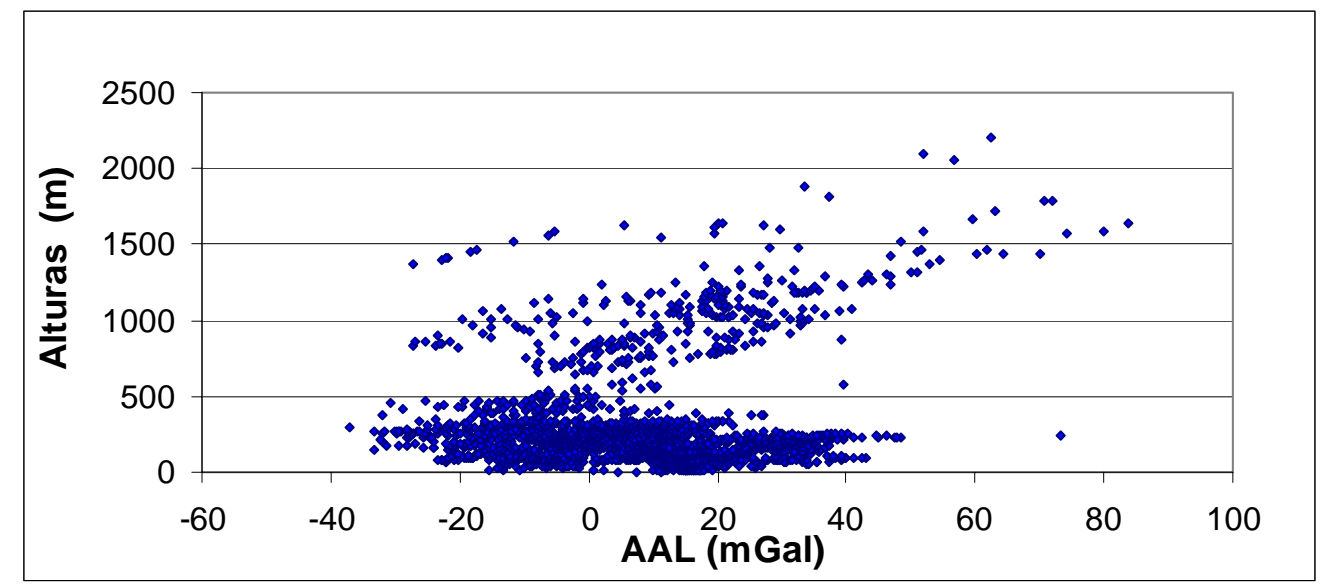

Figura 4.2 - Correlación de las anomalías gravimétricas de aire libre (AAL) con la altura 


\subsubsection{Integración de AAL de datos terrestres con AAL en el mar}

Se integró la información gravimétrica terrestre con anomalías de gravedad de aire libre derivadas de la altimetría satelital en el mar (Andersen et al., 2008), para obtener un mejor conocimiento e interpretación del campo de gravedad terrestre en términos de estructuras geológicas.

Previo a la integración se analizó el juego de datos de anomalías gravimétricas provenientes de la altimetría satelital.

Se usaron los datos del modelo global DNSC08 (Andersen et al., 2008) para el sector marítimo que se ubica en la parte sur de la provincia de Buenos Aires, en el extremo sureste de la zona de estudio.

Los datos obtenidos del modelo están distribuidos en forma de grilla con un espaciado de un minuto y solo abarca la superficie de agua (ver en Figura 4.3). El resumen estadístico de este conjunto de datos se muestra en la Tabla 4.6.

Tabla 4.6.-Anomalías de aire libre en el mar del modelo DNSC08.

\begin{tabular}{l|r}
\hline Num. Nodos & 2994 \\
\hline Valor Máximo de AAL [mGal] & 23 \\
\hline Valor Mínimo de AAL[mGal] & -38 \\
\hline Promedio [mGal] & -8 \\
\hline Desviación estándar [mGal] & \pm 15 \\
\hline
\end{tabular}

Se advierte que los valores de anomalías gravimétricas en el mar del modelo DNSC08 tienen un rango de variación de aproximadamente $60 \mathrm{mGal}$, con mínimos de -38 mGal y máximos de 23 mGal como advierte en la Figura 4.3, en la cual se muestra el grillado de datos de anomalías gravimétrícas clasificadas con una graduación de colores en correspondencia con la variación de valores.

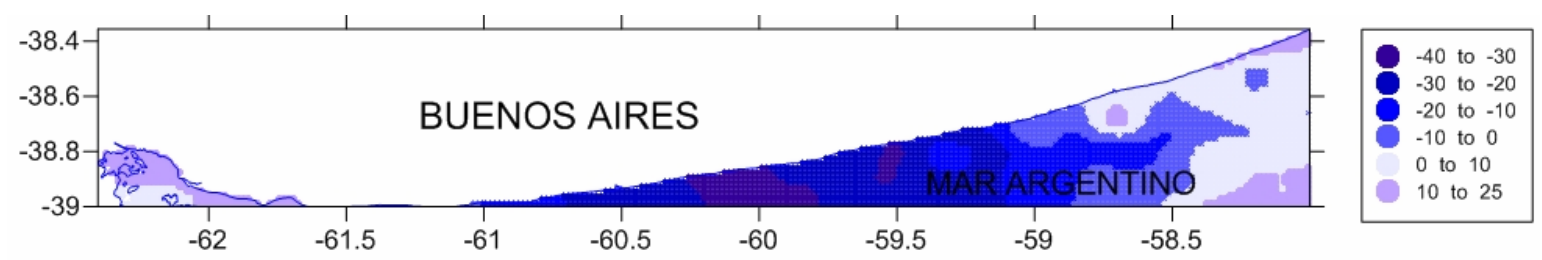

Figura 4.3 - Anomalías gravimétricas del mar provenientes del modelo DNSC08

Para realizar la integración de los datos terrestres IGN con los derivados de la altimetría satelital se generó una grilla con valores interpolados a partir de ambos conjuntos de datos.

Es sabido que en muchos problemas relacionados con las ciencias de la tierra en general y con la geodesia en particular, se dispone de observaciones en puntos discretos del territorio y se requiere estimar los mismos en otros puntos. Para ello es necesario aplicar alguna técnica de 
predicción que produzca el menor error posible en el valor estimado. Una metodología muy aplicada es el método de Kriging, que es similar al método de predicción de colocación por mínimos cuadrados comúnmente usado en geodesia física (Dermanis, 1984, Torge, 1989).

Kriging es una técnica de estimación geoestadística que utiliza una combinación lineal de los valores muestreados para hacer predicciones, aplicando ponderaciones a las observaciones que se determinan a partir de funciones de correlación espacial (semivariogramas) (Sullivan, 1998). Este estimador minimiza la varianza del error (estimador óptimo) y fija el error medio de la predicción en cero (estimador insesgado).

Al conjunto de datos terrestres IGN se adicionó los valores de anomalías gravimétricas DNSC08 derivadas de la altimetría satelital. El nuevo conjunto de datos comprende las anomalías gravimétricas de datos terrestres más las anomalías derivadas de altimetría satelital. La integración se realizó mediante la generación de una grilla con valores de AAL interpoladas a partir de las AAL en puntos IGN y AAL en el mar, aplicando el método geoestadístico de Kriging lineal con una resolución de un minuto tratando de ajustar la resolución del modelo DNSC08. El resumen estadístico de las AAL del modelo integrado (IGN y DNSC08) se muestra en la Tabla 4.7.

Tabla 4.7.- Anomalías de aire libre del modelo integrado con datos IGN y DNSC08

\begin{tabular}{l|r}
\hline Num. Puntos & 138103 \\
\hline Valor Máximo de AAL [mGal] & 80.40 \\
\hline Valor Mínimo de AAL[mGal] & -37.90 \\
\hline Promedio [mGal] & 4.15 \\
\hline Desviación estándar [mGal] & \pm 14.23 \\
\hline
\end{tabular}

En la Figura 4.4.a se muestra el modelo generado a partir de AAL en puntos IGN y en la Figura 4.4.b el modelo de las AAL integrado con datos IGN mas el modelo DNSC08. De las figuras se advierte la mejor solución de las AAL ajustadas a la línea de costa en el modelo integrado. En general los valores de las AAL en el resto de la zona de estudio no se afectaron con la integración. Asimismo, se aprecia la mayor variación de AAL en el sector Oeste, con valores que llegan a los $+80 \mathrm{mGal}$. También se observa variación AAL negativas en el sector Sureste de la provincia de Buenos Aires, en la costa y en una pequeña región central de La Pampa.

Del mismo modo que se trató de analizar la correlación de las AAL con la altura para los puntos IGN mediante una gráfica de dispersión (Figura 4.2), se correlacionó las AAL del modelo integrado con las alturas del modelo digital SRTM30 PLUS. Para ello se superpuso una vista $3 \mathrm{D}$ de las AAL del modelo integrado sobre un mapa topográfico generado a partir del modelo SRTM30 PLUS (Figura 4.5). 
a) AAL a partir de datos IGN

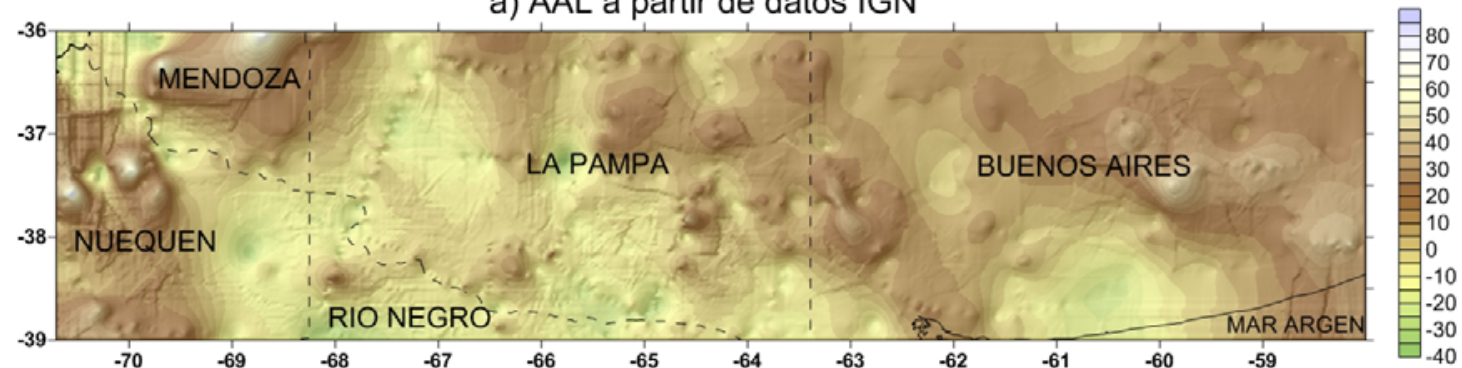

b) AAL integrando datos IGN y DNSC08

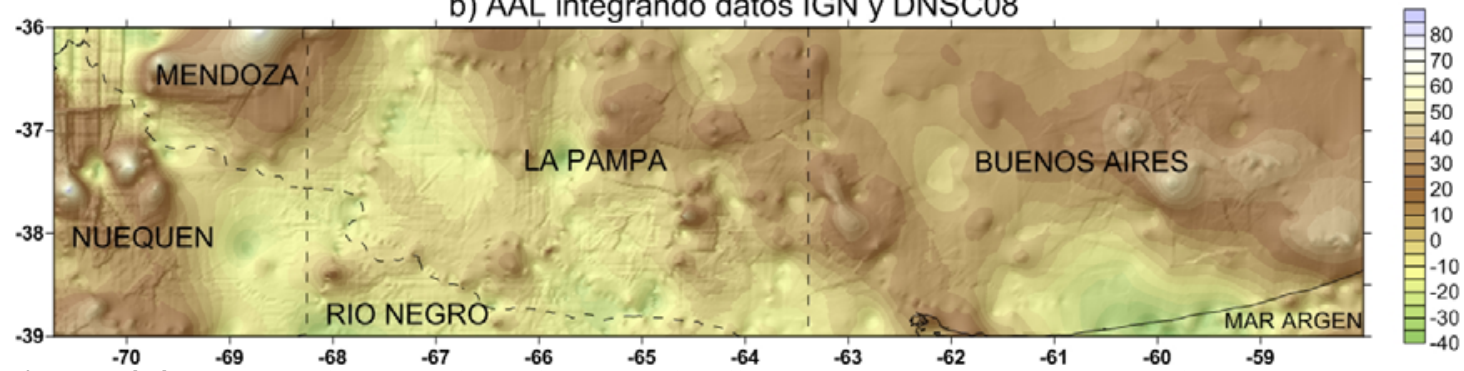

Figura 4.4 - Anomalías gravimétricas de aire libre en mGal. a) AAL a partir de datos IGN. b) AAL del modelo integrado (datos IGN más el modelo DNSC08)

En la Figura 4.5 se advierte una correlación general de las AAL con la altura. Hacia el oeste las mayores alturas están vinculadas con mayores valores de AAL. Se advierte AAL negativas en el sector marítimo y también en algunos sectores en zona de montaña y serranías. Un comportamiento particular de AAL positivas se advierte en el sector Este, en el sistema de Tandilia, donde las alturas no son tan elevadas para justificar altos valores de AAL.

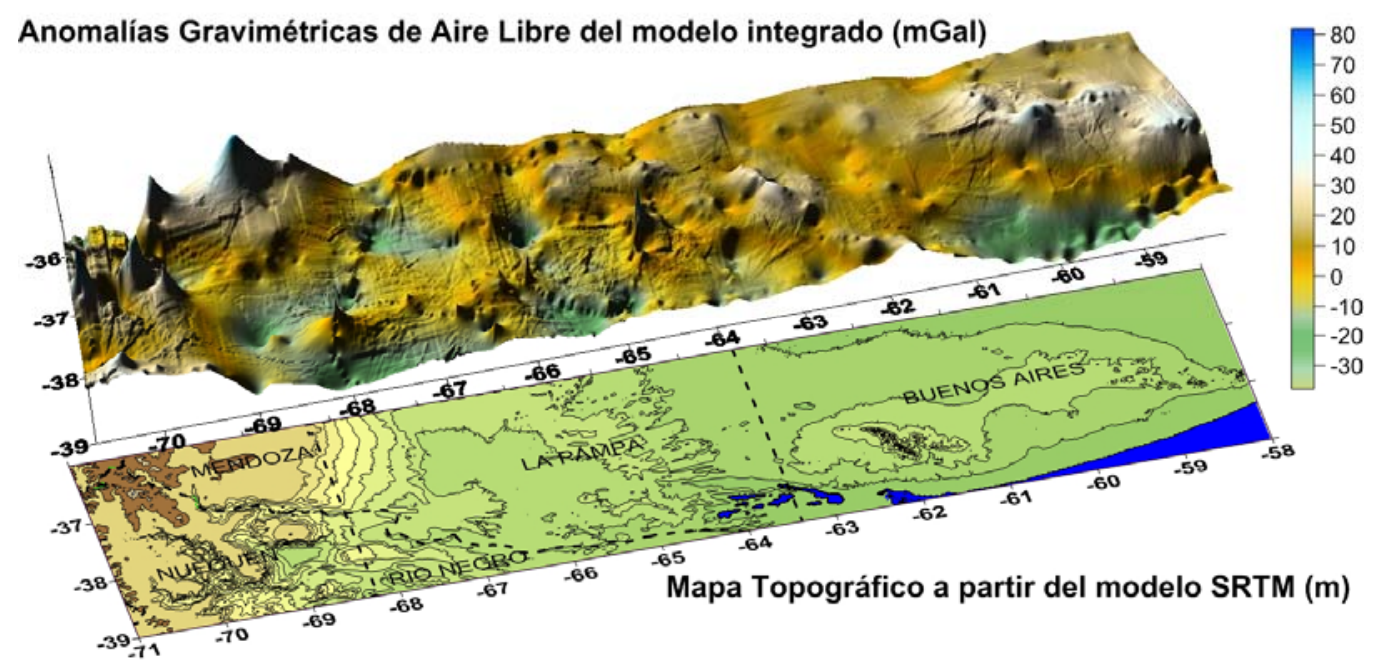

Figura 4.5 -. Anomalías gravimétricas de aire libre del modelo integrado superpuesto sobre mapa topográfico obtenido del modelo SRTM30 PLUS. Unidades anomalías aire libre en mGal

Con el objetivo de analizar la variación de las AAL de los datos provenientes del mar al ser integrados a los datos terrestres se hizo un cálculo de diferencias o residuos entre la grilla obtenida de la integración y los datos DNSC08 originales. Los residuos dieron valores bajos 
como se advierte en el resumen estadístico que se muestra a continuación en la Tabla 4.8. El $99 \%$ de los residuos están comprendidos entre $\pm 2 \mathrm{mGal}$. Un solo punto toma un valor de +2.9 mGal que se muestra en color magenta oscuro en la Figura 4.6.

Tabla 4.8.- Estadísticos de los residuos de anomalías de aire libre del modelo DNSC08 con el modelo integrado (datos terrestres y marinos).

\begin{tabular}{l|r}
\hline Num. Nodos & 2994 \\
\hline Valor Máximo de Residuos AAL [mGal] & 2.89 \\
\hline Valor Mínimo de Residuos AAL[mGal] & -1.82 \\
\hline Promedio Residuos [mGal] & 0.01 \\
\hline Desviación estándar Residuos [mGal] & \pm 0.18 \\
\hline
\end{tabular}

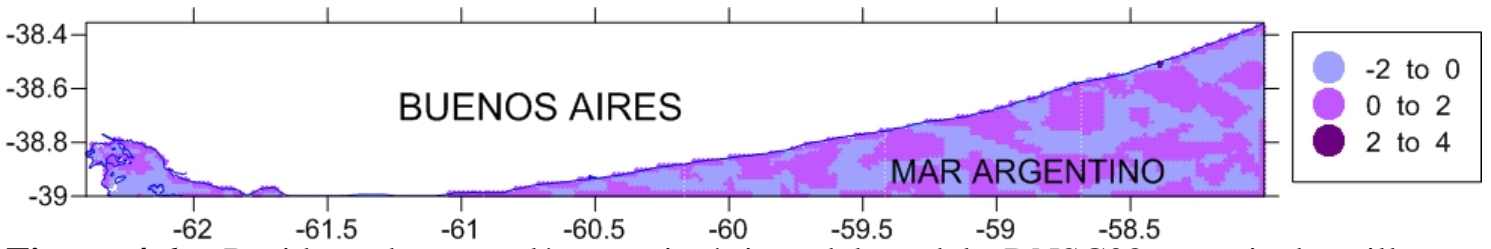

Figura 4.6 - Residuos de anomalías gravimétricas del modelo DNSC08 a partir de grilla con datos terrestre y marinos

\subsection{Anomalía Gravimétrica de Bouguer}

Se han calculado las anomalías de gravedad de Bouguer, utilizando el modelo clásico de placa de Bouguer y el modelo de capa esférica (LaFehr, 1991) de acuerdo a las ecuaciones (2.15) y (2.18) respectivamente. Asimismo se obtiene la anomalía de Bouguer completa con la ecuación (2.19) usando por un lado la corrección topográfica obtenida con el programa Tc2DFT (Li, 1993) que usa la transformada de Fourier.

Para el cálculo de las anomalías de Bouguer completa se determinaron las correcciones topográficas con el modelo digital de elevación SRTM30 PLUS en una grilla de 30 segundos de arco ( 1 kilómetro de resolución espacial).

En todos los resultados numéricos de las anomalías de Bouguer y de las correcciones topográficas, presentados en este capítulo, se utilizó una densidad constante de $2.67 \mathrm{~g} / \mathrm{cm}^{3}$, la cual es convencionalmente usada y corresponde a la densidad del granito (Torge, 1989).

\subsubsection{Anomalía de la Gravedad de Bouguer Plana Simple (ABPS)}

La anomalía de Bouguer plana simple (ABPS) se calculó de acuerdo con la ecuación (2.15), donde $H_{P}$ es la altura del punto de gravedad medida, es decir los puntos de nivelación IGN. El resumen estadístico de este cálculo se muestra en la Tabla 4.9. 
Las ABPS tienen un rango de variación entre $-183.88 \mathrm{mGal}$ a $45.78 \mathrm{mGal}$. Los valores fuertemente negativos están asociados a la presencia de topografía elevada como se aprecia en la Figura 4.7.a, en la cual se advierte la tendencia que a mayores alturas se corresponden las AB más negativas.

Tabla 4.9.-Anomalías de Bouguer para los puntos IGN

\begin{tabular}{l|r|r|r|r|r}
\hline & \multicolumn{1}{|c|}{ Min. } & \multicolumn{1}{c|}{ Máx. } & \multicolumn{1}{l}{ Rango } & \multicolumn{1}{l}{ Media } & \multicolumn{1}{c}{ Desv. Est. } \\
\hline Anomalía de Bouguer Plana Simple [mGal] & -183.88 & 45.78 & 229.66 & -26.06 & \pm 36.49 \\
\hline Anomalía de Bouguer Esférica Simple [mGal] & -182.37 & 46.12 & 228.49 & -25.69 & \pm 36.20 \\
\hline Corrección Topográfica [mGal] & 0.003 & 8.15 & 8.153 & 0.21 & \pm 0.59 \\
\hline Anomalía de Bouguer Plana Completa [mGal] & -180.95 & 45.90 & 226.85 & -25.85 & \pm 36.07 \\
\hline
\end{tabular}

Para analizar la estructura espacial de las ABPS se generó una grilla para toda la zona de estudio con un espaciado de un minuto de arco, usando el método de interpolación Kriging lineal. La variación espacial de las ABPS se muestra en la Figura 4.8.a. En la misma se advierte claramente la correlación negativa de las anomalías de Bouguer con la altura, donde los mayores valores negativos se ubican hacia el oeste en correspondencia con el sistema de Los Andes.

\subsubsection{Anomalía de la gravedad de Bouguer esférica simple (ABES)}

La anomalía de Bouguer esférica simple (ABES) se calculó según la ecuación (2.18). Las ABES tienen un rango de variación entre -182.37 mGal a $46.1 \mathrm{mGal}$ como se muestra en la Tabla 4.9. Al comparar estos valores con los de las ABPS se advierte un comportamiento similar aunque con magnitudes menos negativas. En la Figura 4.7.b, se aprecia la misma tendencia que a mayores alturas se corresponden las ABES más negativas.

De igual modo que para las ABPS, se generó una grilla de ABES para la zona de estudio, con resolución de un minuto de arco ( $\sim 2 \mathrm{~km}$ de resolución espacial) usando el método de interpolación Kriging lineal. La variación espacial de ABES se muestra en la Figura 4.8.b, con valores fuertemente negativos en relación con las mayores alturas, comportamiento similar a las ABPS. 


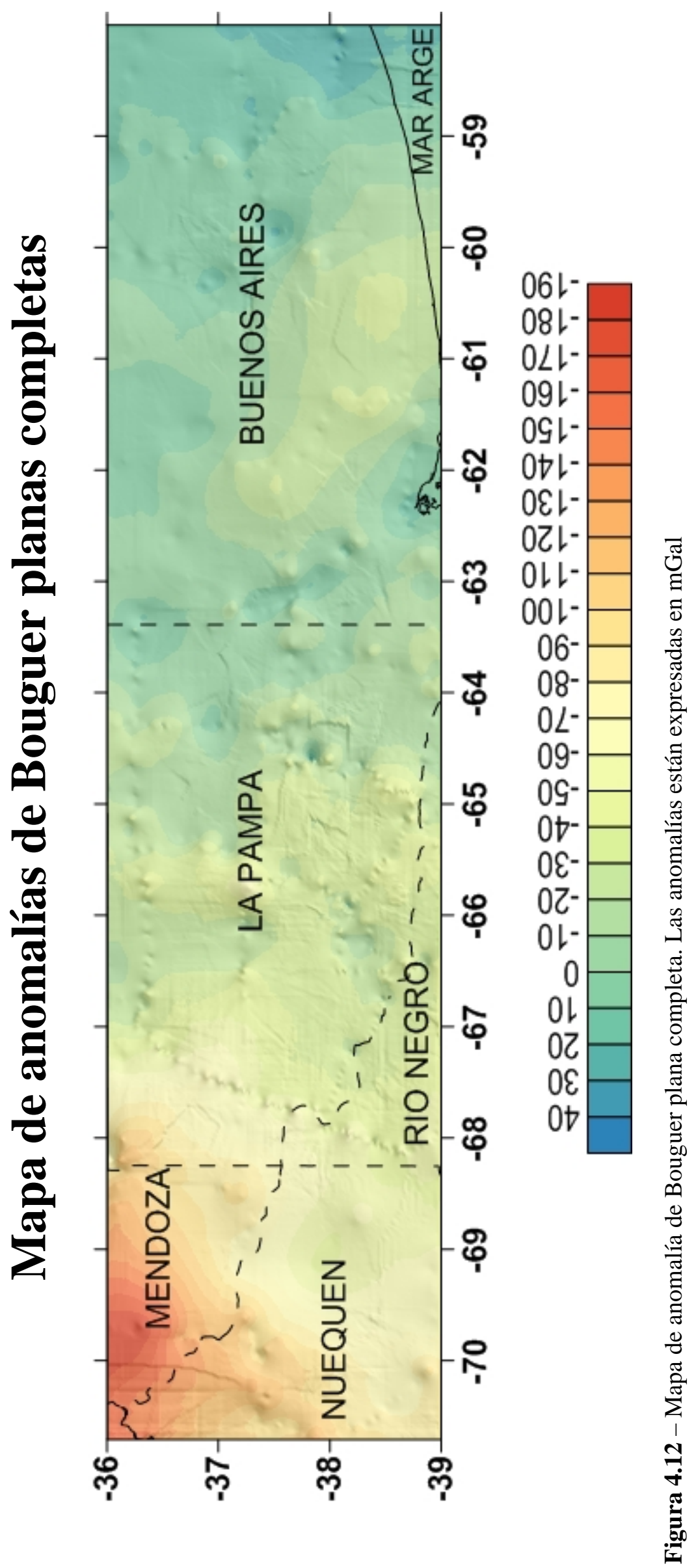


Con el objeto de tener una evaluación de las discrepancias de las ABPC frente a los modelos obtenidos previamente (ABPS y ABES), se realizó la diferencia entre ellas, generándose una grilla como diferencia entre la grilla de ABPC menos las grillas de ABPS y de ABES. A partir de estas diferencias se realizó el análisis estadístico en los nodos de grilla (Tabla 4.10). El rango de variación de las diferencia de las ABPC con las ABPS es superior que con las ABES, lo cual muestra que las anomalías de Bouguer simples en el modelo esférico son más consistentes que las del modelo planar con las anomalías de Bouguer planas completas. Esto último también lo demuestra la menor magnitud del promedio y la desviación estándar de las diferencias (Tabla 4.10).

Tabla 4.10.- Diferencias entre las ABPC y las ABPS y ABES.

\begin{tabular}{l|c|c|}
\hline & ABPC-ABPS & ABPC-ABES \\
\hline Núm. Nodos & 138103 & 138103 \\
\hline Mínimo [mGal] & 0.00 & -0.87 \\
\hline Máximo[mGal] & 7.21 & 5.94 \\
\hline Rango [mGal] & 7.21 & 6.81 \\
\hline Promedio [mGal] & 0.30 & -0.12 \\
\hline Desviación estándar [mGal] & \pm 0.69 & \pm 0.46 \\
\hline
\end{tabular}
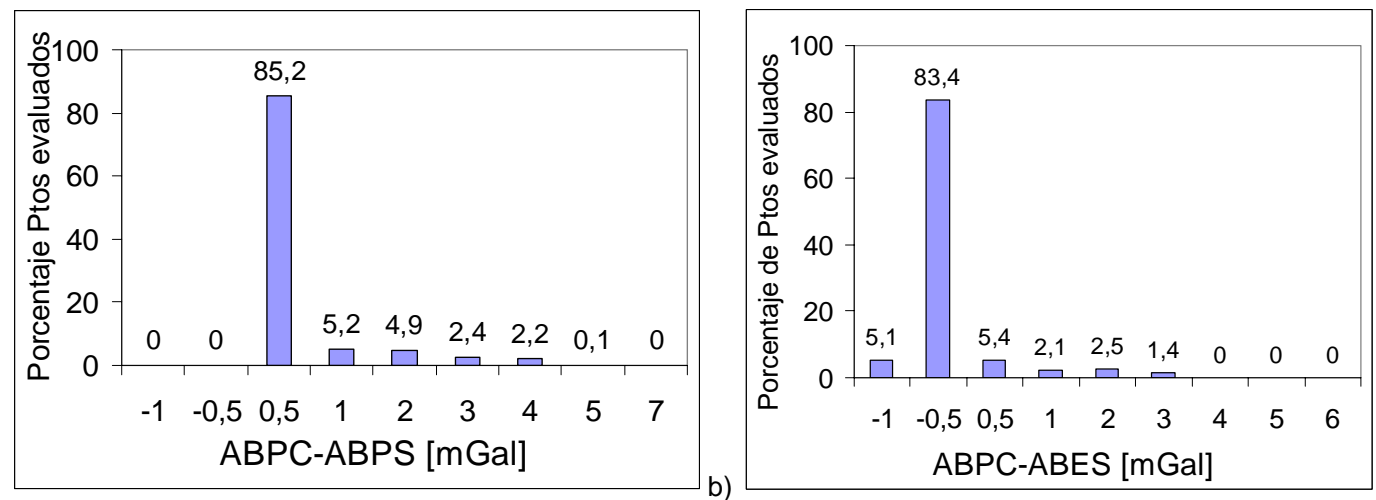

Figura 4.13 - Diferencias entre las diferentes anomalías de Bouguer calculadas. a) Diferencia entre ABPC y ABPS. b) Diferencia entre ABPC y ABES

Al comparar la ABPC con la ABPS, se advierte que el 85\% de puntos evaluados (nodos de la grilla) tiene una diferencia en el intervalo de 0 a $0.5 \mathrm{mGal}$ (Figura 4.13.a). Este resultado sugiere por un lado un buen ajuste entre ambos modelos, y por otro, el valor positivo de las diferencias muestra el efecto gravitacional de la corrección por topografía siempre positiva en el modelo planar.

Asimismo al analizar la ABPC con la ABES, se advierte que el 83\% de los puntos evaluados tienen una diferencia entre 0 a $-0.5 \mathrm{mGal}$ (Figura 4.13.b). Este resultado sugiere también un buen ajuste entre ambos modelos, en tanto que el valor negativo de las diferencias 
sugiere que el efecto gravitacional de la capa esférica es mayor sobre la placa plana de Bouguer con corrección por topografía.

La estructura espacial de las diferencias se muestra en la Figura 4.14. En la misma se advierte que las mayores discrepancias se encuentran en la zona Oeste donde la topografía es más movida.

\section{a) ABPC - ABPS}

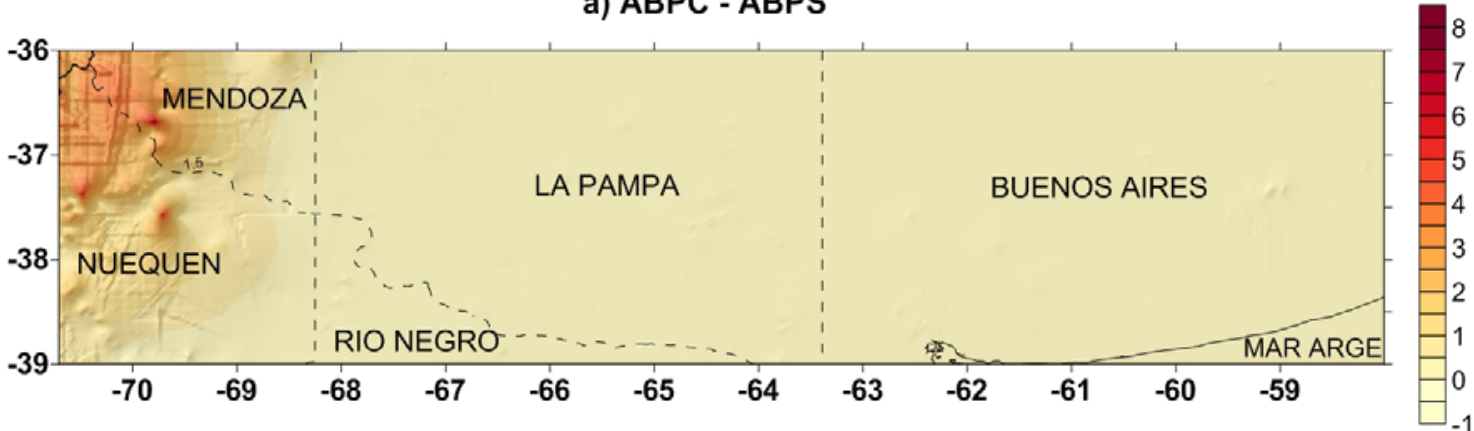

b) ABPC - ABES

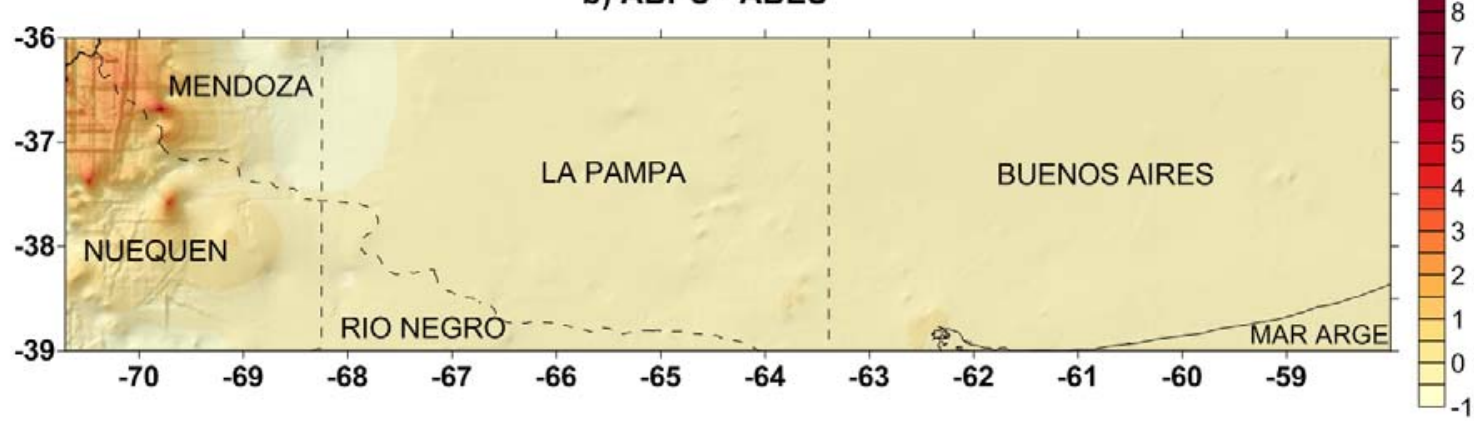

Figura 4.14 - Anomalías de Bouguer residual. (a) ABPC-ABPS (b) ABPC-ABES. Todas las anomalías están expresadas en mGal

El análisis de las diferencias de la anomalía de Bouguer plana completa con la anomalía de Bouguer simple, tanto en el modelo plano como esférico son consistentes entre sí por debajo de los $\pm 0.5 \mathrm{mGal}$ en un gran porcentaje en zona de topografía suave. Para zonas de topografía rugosa las discrepancias crecen con ambos modelos, no obstante en el modelo plano las discrepancias son mayores.

\subsection{Anomalías gravimétricas para la localización de estructuras}

Para la localización de las estructuras locales en base a anomalías gravimétricas en tierra (con longitud de onda corta), los mapas de anomalías de Bouguer son útiles (siempre que se aplique la densidad correcta), ya que las $\mathrm{AB}$ son aproximadamente iguales a cero. En los mapas de aire libre, las anomalías locales serían enmascaradas por los efectos de la topografía.

Sin embargo la situación es diferente en el mar, para las anomalías de Bouguer en el mar, el agua se sustituye por material rocoso y por lo tanto el gran término positivo de la 
corrección de Bouguer enmascara las estructuras locales. Por lo tanto, para muchas aplicaciones geofísicas es útil el uso de los mapas de Bouguer en la tierra y los mapas de aire libre en el mar.

\subsection{Mapas de anomalías gravimétricas}

Con los resultados de anomalías gravimétricas obtenidas en este capítulo se ha confeccionado un mapa integrando las anomalías gravimétricas de Bouguer en tierra y las anomalías de aire libre en el mar, como se muestra en la Figura 4.15.

Se ha escogido la anomalía de Bouguer plana completa (ABPC) por ser la variable que toma en cuenta las anomalías de masa de acuerdo a las particularidades de la topografía de la zona de estudio. En cuanto a la zona de mar se ha tomado el mapa integrando de datos terrestres IGN y datos DNSC08 en el mar. Esto último se decidió para evitar problemas de borde en la línea de costa.

En la Figura 4.15 se advierte que las ABPC son muy negativas en el sector Noroeste, en la provincia de Mendoza (las de mayor magnitud se encuentran hacia el Oeste) y positivas en el sector este en la provincia de Buenos Aires.

En el mar la situación es la siguiente: valores de AAL positivas en el Este y negativos en la zona Centro Sur.

Se ha confeccionado también el mapa de anomalías de aire libre integrando datos terrestres IGN y datos marinos DNSC08 (ver Figura 4.16)

\subsection{Conclusiones del capítulo}

En este capítulo se han obtenido las anomalías de aire libre (AAL) integrando datos terrestres del IGN y anomalías gravimétricas en el mar del modelo DNSC08.

También se han determinado anomalías de Bouguer planas y esféricas simples, y las anomalías de Bouguer planas completas o refinadas. Se ha comparado la anomalía de Bouguer plana completa (ABPC) con las anomalías de Bouguer simples tanto en el modelo plano como esférico. Los resultados muestran que para zonas con cambios de elevación moderada, que corresponde a gran parte del área de trabajo, la elección de uno u otro enfoque es de menor importancia. Sin embargo en la zona de topografía rugosa (al Oeste de la zona de trabajo) se recomienda la selección de las anomalías de Bouguer planas completas. 


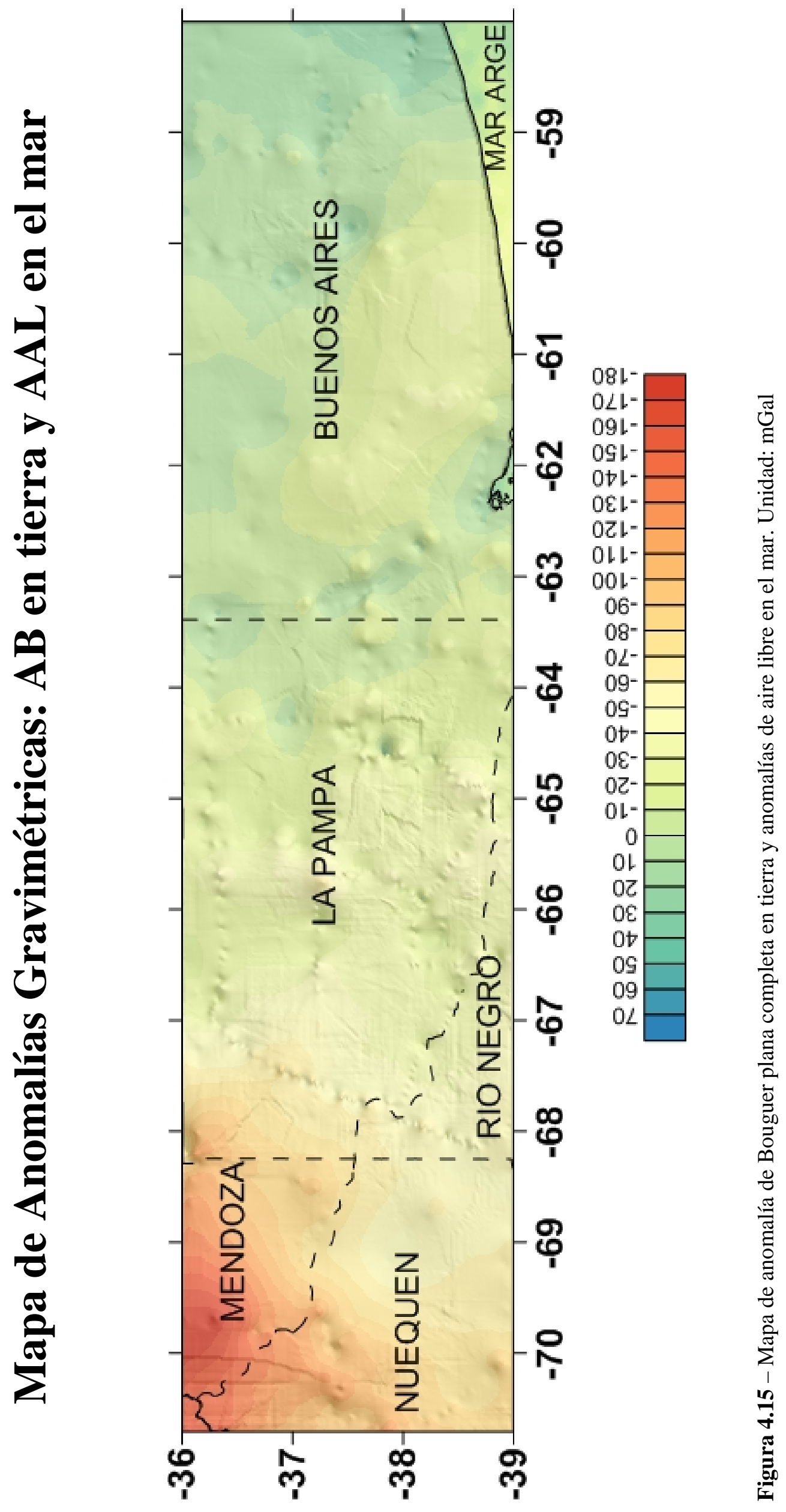




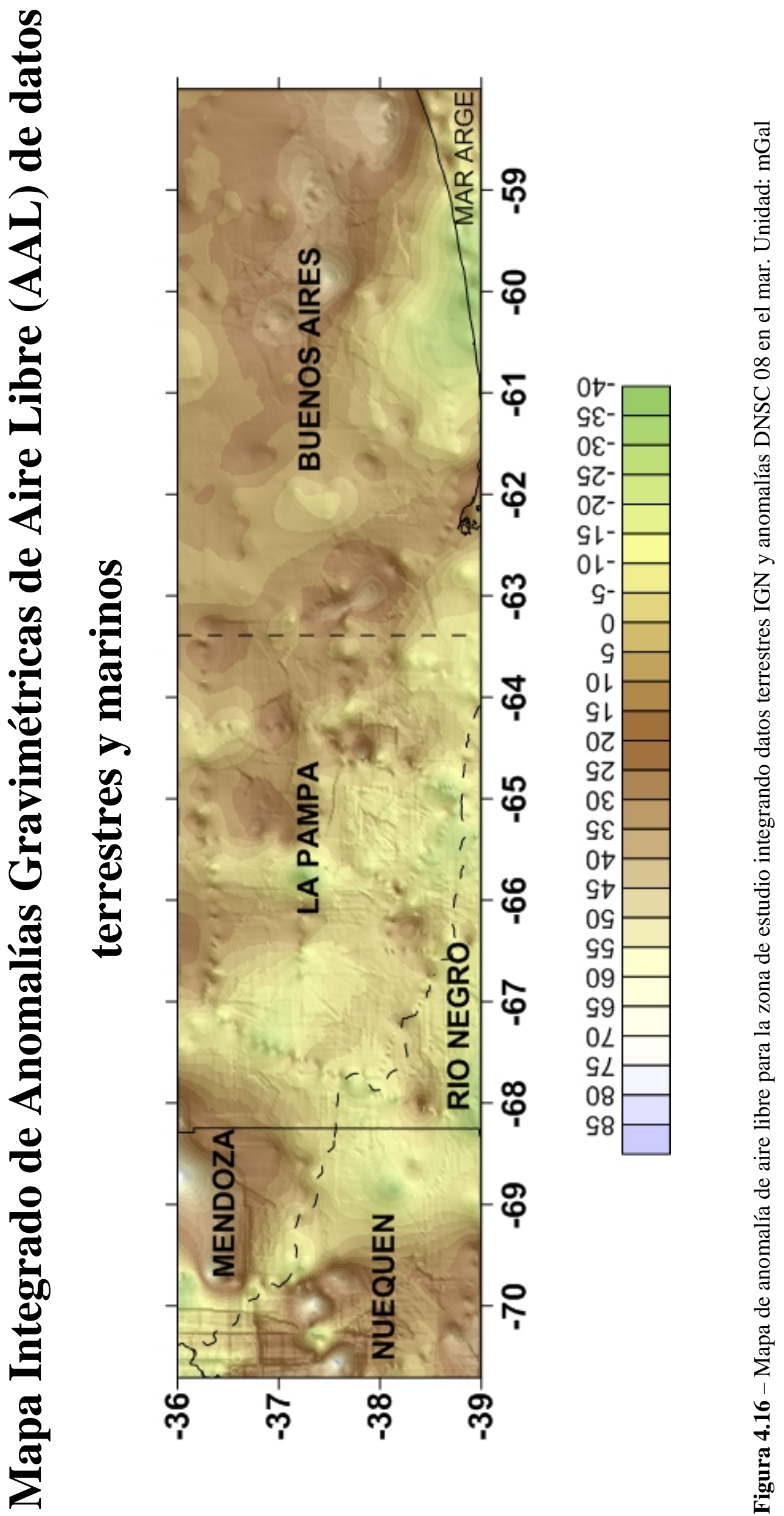




\section{Capítulo V}

\section{Evaluación de Resultados}

En este capítulo se comparan las anomalías de aire libre (AAL) y las anomalías de Bouguer planas simples (ABPS) obtenidas en el Capítulo IV con las obtenidas a partir del modelo geopotencial EGM2008 (Pavlis et al., 2008), por ser el modelo global de mejor resolución espacial y de libre disponibilidad hasta la fecha. La ABPS es la magnitud que se obtiene desde el calculador del ICGEM, por ello su selección a los fines de la comparación.

Asimismo debemos considerar que los modelos geopotenciales globales usan diferentes modelos digitales de elevación para el cálculo de sus reducciones. En particular el modelo EGM2008 usa el modelo digital de elevación DTM2006. A fin de evaluar las alturas usadas en el cálculo de las anomalías gravimétricas en el capítulo anterior y las del modelo global se analizaron las diferencias entre ellos.

\subsection{Evaluación del modelo digital de elevación DTM2006 en puntos discretos IGN}

Las alturas del modelo DTM2006 están referidas al modelo de geoide del EGM2008. Para evaluar las diferencias con las alturas IGN usadas en el cálculo de las AAL, se realizó el cálculo de los desvíos con la ecuación (5.1):

$$
\text { Dif .Altura }{ }_{\text {IGN-DTM 2006 }}=\text { Altura }_{\text {IGN }}-\text { Altura }_{\text {DTM 2006 }} \text {. }
$$

El resumen estadísticos de las diferencias se muestra en la Tabla 5.1, en la segunda columna.

Tabla 5.1.- Evaluación de modelo digital de elevación global en puntos discretos. Diferencias entre alturas IGN y alturas DTM2006 y SRTM30 PLUS, respectivamente.

\begin{tabular}{l|l|l}
\hline & IGN - DTM2006 & IGN - SRTM30 PLUS \\
\hline Num. puntos & 2429 & 2429 \\
\hline Valor Máximo Altura [m] & 755.54 & 861.82 \\
\hline Valor Mínimo Altura [m] & -318.98 & -384.87 \\
\hline Promedio [m] & -3.92 & -0.96 \\
\hline Desviación estándar [m] & 49.66 & 51.10 \\
\hline
\end{tabular}


Las diferencias en relación con la altura mostradas en la Figura 5.1 revelan que las mayores discrepancias corresponden a mayores alturas, donde la topografía refleja fuertes pendientes. Como se mencionó en el Capítulo III, la georreferenciación de los puntos de nivelación IGN tiene una exactitud variable, y en zonas de topografía abrupta, con pendientes elevadas, este aspecto es crítico. Por otro lado, los métodos de relevamiento de la topografía global también son menos precisos en lugares con pendientes empinadas (Farr et al., 2007; Rodríguez et al., 2005).
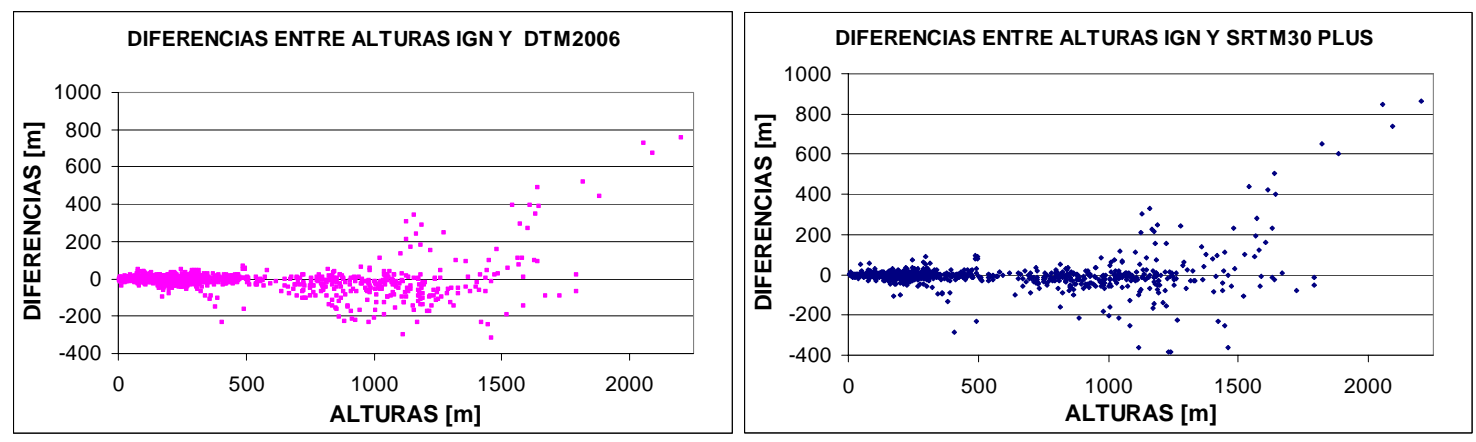

Figura 5.1.- Diferencia entre alturas IGN y alturas modelo digital de elevación. Izquierda: diferencias del modelo DTM2006 en relación a la altura IGN. Derecha: diferencias del modelo SRTM30 PLUS en relación a la altura IGN

En la Figura 5.1. Izquierda se observa que para alturas superiores a $1000 \mathrm{~m}$ los desvíos superan los $200 \mathrm{~m}$, mientras que para alturas mas bajas el ajuste es mejor que $100 \mathrm{~m}$ en la mayoría de los casos. La Figura 5.2. Izquierda muestra que el 96\% de los puntos evaluados tienen una diferencia de alturas menor que $100 \mathrm{~m}$. Este comportamiento de las alturas es importante como fuente de error en el cálculo de las anomalías gravimétricas.
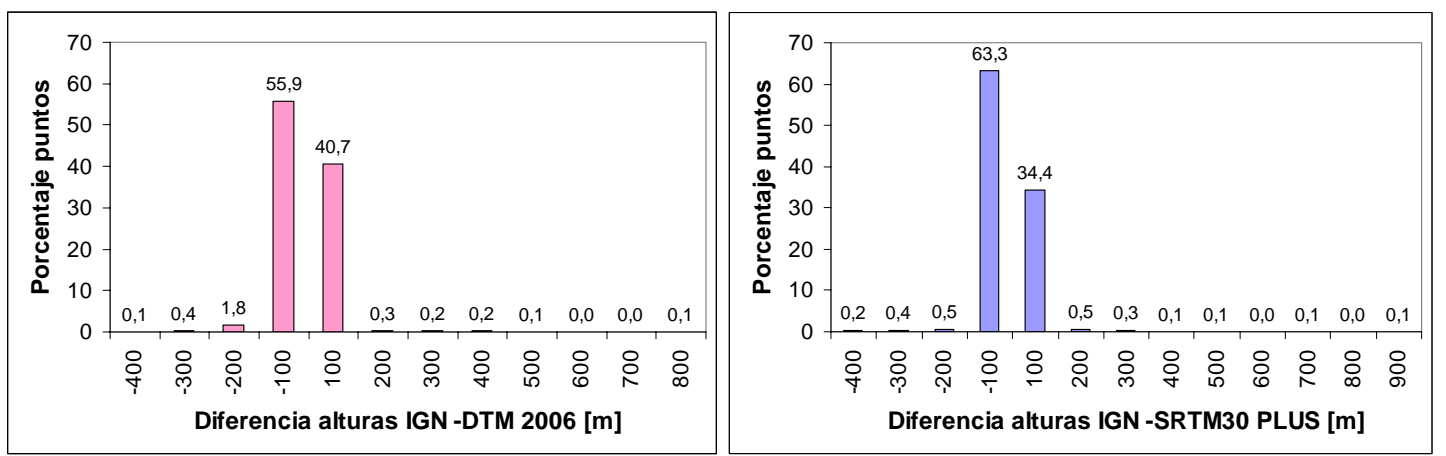

Figura 5.2.- Evaluación de diferencias de alturas entre puntos IGN y modelo digital de elevación. Izquierda: con modelo DTM2006. Derecha: con modelo SRTM30 PLUS 


\subsection{Evaluación del modelo digital de elevación SRTM30 PLUS en puntos discretos IGN}

Las alturas del modelo SRTM30 PLUS están referidas al modelo de geoide del EGM96. El modelo SRTM30 PLUS se usó en el cálculo de las correcciones topográficas planas para obtener la anomalía de Bouguer refinadas. Para evaluar las diferencias con las alturas IGN usadas en el cálculo de las AAL, se realizó el cálculo de los desvíos de manera análoga a la usada en el modelo DTM2006.

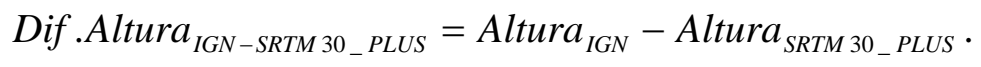

El resumen estadístico de las diferencias se muestra en la Tabla 5.1 en la tercera columna. Se advierte que el promedio de las diferencias es menor que el metro y su desviación estándar de aproximadamente $50 \mathrm{~m}$. En la Figura 5.1. Derecha, se advierte de igual manera que las mayores diferencias corresponden a mayores alturas donde como ya lo expresamos las pendientes son mayores. También se advierte en la Figura 5.2. Derecha que un alto porcentaje de puntos evaluados (casi el $98 \%$ ) es menor que $100 \mathrm{~m}$. Esto sugiere un mejor ajuste de las alturas del modelo SRTM30 PLUS frente al modelo DTM2006.

En el cálculo de la corrección topográfica realizado en el Capítulo IV se advirtió que para alturas menores de $500 \mathrm{~m}$ el valor de ésta corrección no supera $1 \mathrm{mGal}$. Sin embargo este valor aumenta hasta una magnitud de $8 \mathrm{mGal}$ para mayores alturas, donde también se encuentran las mayores diferencias entre las alturas IGN y SRTM30 PLUS.

\subsection{Comparación entre DTM2006 y SRTM30 PLUS}

Asimismo se realizó la comparación entre los modelos DTM2006 y SRTM30 PLUS. Para ello se realizó la diferencia entre las grillas de ambos modelos con resolución de un minuto, con la ecuación (5.3):

$$
\text { Dif .Altura } a_{S R T M-D T M}=\text { Altura }_{\text {SRTM 30_PLUS }}-\text { Altura }_{\text {DTM } 2006} \text {. }
$$

El resumen estadístico de estas diferencias se muestra en la Tabla 5.2 tercera columna. La estructura espacial de los modelos y sus diferencias se muestra en la Figura 5.4.

Los estadísticos de las alturas en los nodos de la grilla de ambos modelos y la diferencia de alturas entre ambos, se resumen en la Tabla 5.2. Se advierte que las alturas del DTM2006 llegan a un máximo de 3727 m, en cambio las alturas del SRTM30 PLUS llegan a 4557 m, esto justifica que la magnitud de las diferencias llegue a un valor de $927 \mathrm{~m}$. Estas diferencias se 
manifiestan en el sector de mayores pendientes que es en el sector Oeste, como se puede advertir en la Figura 5.4.

Tabla 5.2.- Resumen estadístico de alturas DTM2006, SRTM30 PLUS y sus diferencias.

\begin{tabular}{l|l|l|l}
\hline & DTM2006 & SRTM30 PLUS & Diferencia \\
\hline Num. Nodos Grilla & 141361 & 141361 & 141361 \\
\hline Valor Máximo altura [m] & 3727.47 & 4557.92 & 927.78 \\
\hline Valor Mínimo altura [m] & -82.80 & -83.04 & -700.15 \\
\hline Promedio [m] & 424.32 & 424.88 & 0.55 \\
\hline Desviación estándar [m] & \pm 527.36 & \pm 529.26 & \pm 62.56 \\
\hline
\end{tabular}

El rango del las diferencias es de 1628 m, no obstante el mayor porcentaje (casi el 94\%) de puntos evaluados (nodos de grilla) tienen diferencias por debajo de los $\pm 100 \mathrm{~m}$ como lo demuestra la Figura 5.3.

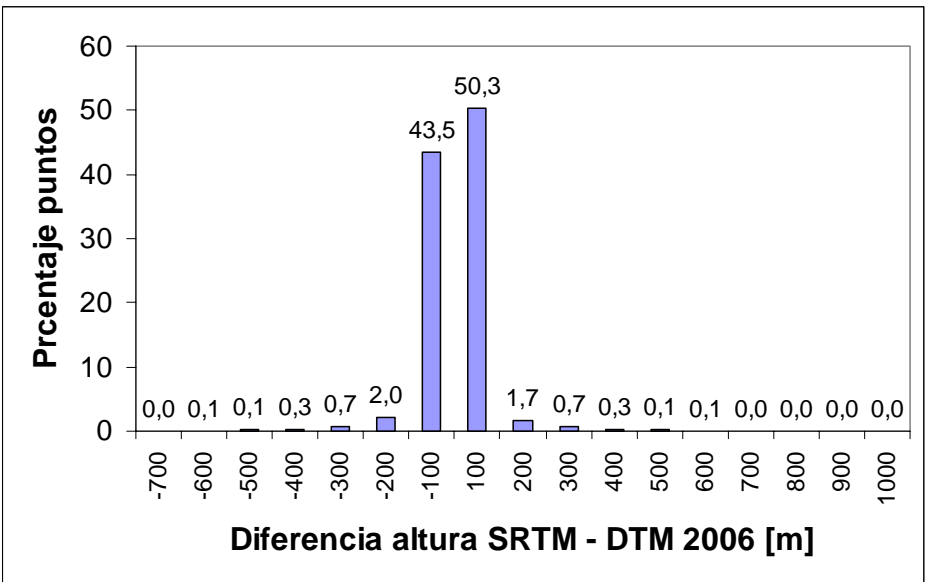

Figura 5.3.- Evaluación de diferencias de alturas entre modelos SRTM30 PLUS y DTM2006

Si bien en este cálculo ambos modelos de elevación están grillados con la resolución de un minuto, en la Figura 5.4 se advierte que el modelo SRTM30 PLUS representa mejor las particularidades de la topografía. Esto se debe a la mayor resolución espacial de este modelo, que es de 30 segundos de arco, es decir aproximadamente $1 \mathrm{~km}$ en la superficie terrestre. En cuanto al modelo DTM2006 la resolución del mismo es de 5 minutos de arco, aproximadamente $9 \mathrm{~km}$ en la superficie terrestre, lo que se advierte en las formas más suaves para representar la topografía abrupta, en particular el sistema de Los Andes al Oeste. 


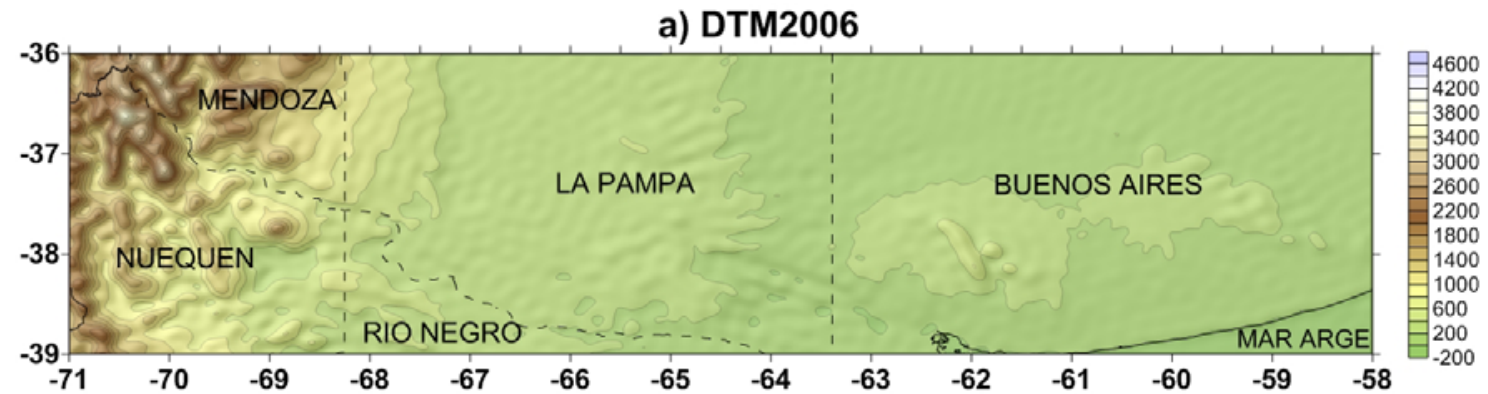

b) SRTM30 PLUS

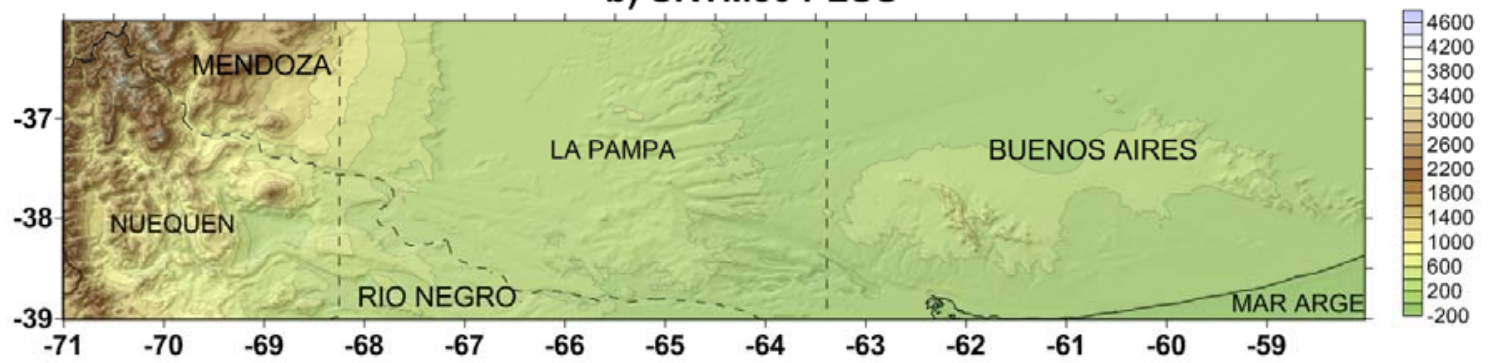

c) DIFERENCIA SRTM30 PLUS - DTM 2006

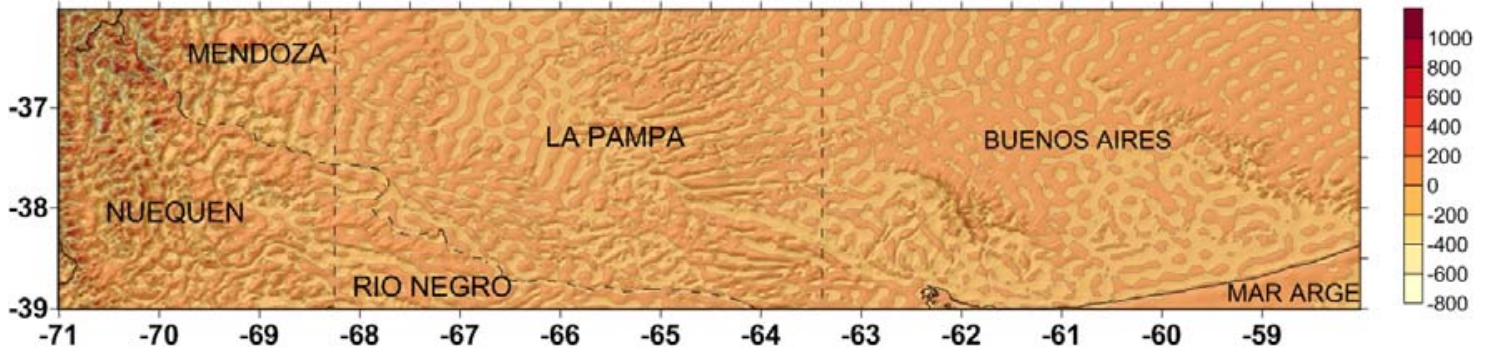

Figura 5.4.- Comparación entre modelos digitales de elevación. a) Modelo DTM2006. b) Modelos SRTM30 PLUS c) Diferencia

\subsection{Influencia de la altura en el cálculo de anomalías gravimétricas}

Las diferencias entre alturas de los modelos digitales de elevación DTM2006 y SRTM30 PLUS producen diferencias en las reducciones para el cálculo de anomalías gravimétricas.

De acuerdo a la Figura 5.2. Izquierda casi el $97 \%$ de los puntos IGN tienen una diferencia de altura con el modelo DTM2006 menor a \pm 100 m. En el cálculo de la corrección de aire libre $(0.3086 \times \mathrm{H})$, un error de $100 \mathrm{~m}$ ocasionaría un desvío de $30.86 \mathrm{mGal}$ en la anomalía gravimétrica de aire libre. En un valor máximo de diferencia de altura de 1000 m produciría un desvío en la anomalía gravimétrica de 308 mGal. 


\subsection{Anomalía de aire libre del modelo geopotencial EGM2008 y su comparación con las determinadas con datos IGN más datos DNSC08}

Se obtuvieron las anomalías de aire libre (AAL) del modelo geopotencial EGM2008 en la región de estudio, en una grilla regular con resolución de un minuto, resolución similar a la grilla obtenida en el Capítulo IV a partir de datos terrestres IGN más anomalías gravimétricas DNSC08 en el mar.

El resumen estadístico de los valores AAL del modelo global y del modelo integrado obtenido anteriormente a partir de datos terrestres IGN y en el mar datos DNSC08, se muestra en la Tabla 5.3.

Tabla 5.3.-Anomalías de aire libre del modelo integrado (IGN+DNSC08), del modelo EGM2008 y sus diferencias.

\begin{tabular}{l|l|l|l}
\hline & EGM2008 & IGN + DNSC08 & Diferencia \\
\hline Num. Nodos Grilla & 138103 & 138103 & 138103 \\
\hline Valor Máximo de AAL [mGal] & 338.42 & 80.40 & 336.05 \\
\hline Valor Mínimo de AAL[mGal] & -56.40 & -37.90 & -82.07 \\
\hline Promedio [mGal] & 10.45 & 4.15 & 4.70 \\
\hline Desviación estándar [mGal] & \pm 27.35 & \pm 14.23 & \pm 23.72 \\
\hline
\end{tabular}

El rango de valores AAL del EGM2008 es de $394.82 \mathrm{mGal}$, es tres veces superior al rango de AAL del modelo integrado que es de $118.30 \mathrm{mGal}$.

Para analizar el comportamiento de ambos modelos y sus variaciones, se realizó la diferencia entre ellos mediante la ecuación (5.4):

$$
\text { Dif . } A A L=A A L_{E G M 2008}-A A L_{\text {mod.int egr (IGN+DNSC 08) }} \text {. }
$$

El resultado de esta diferencia es otra grilla con la misma resolución de los dos modelos anteriores y cuya variable espacial es la diferencia de AAL entre ambos. El resumen estadístico de las diferencias se muestra en la Tabla 5.3. El rango de las diferencia de AAL entre el modelo global y el modelo integrado es de $418 \mathrm{mGal}$, sin embargo el 94 \% de los puntos tienen una diferencia menor de +/- $30 \mathrm{mGal}$. Esto se corresponde con las diferencias determinadas entre las alturas IGN y las alturas del modelo DTM2006, ya que como mencionamos anteriormente, 97\% de los puntos tienen una diferencia de altura menor que $100 \mathrm{~m}$ lo que produciría un error de 31 mGal. 


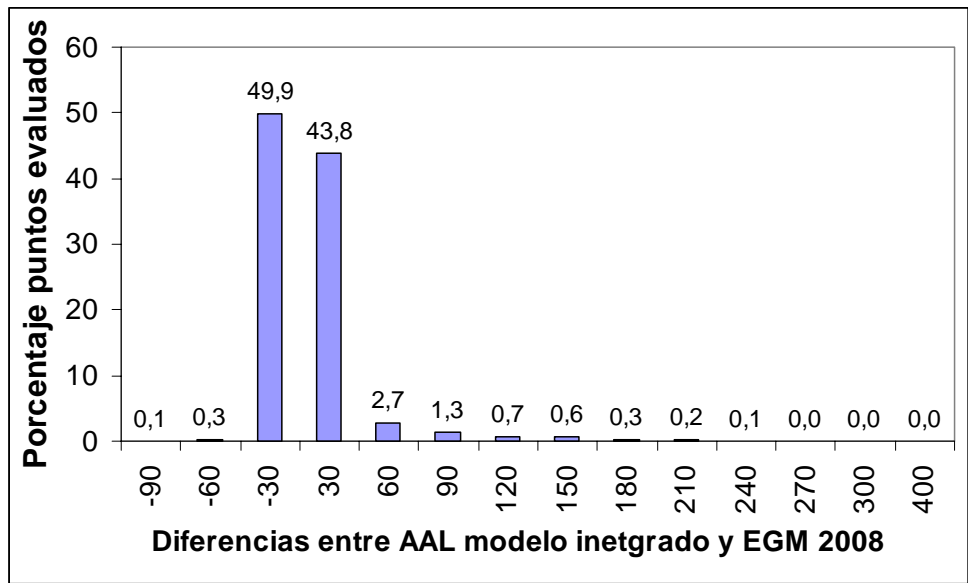

Figura 5.5.- Evaluación de diferencias de anomalías de aire libre entre el modelo integrado (IGN+DNSC08) y el EGM2008

Para visualizar la estructura espacial de ambos modelos de AAL y sus diferencias se confeccionaron los mapas de anomalías, que se muestran en la Figura 5.6.

a) Anomalías aire libre (AAL) modelo integrado (IGN + DNSC08)

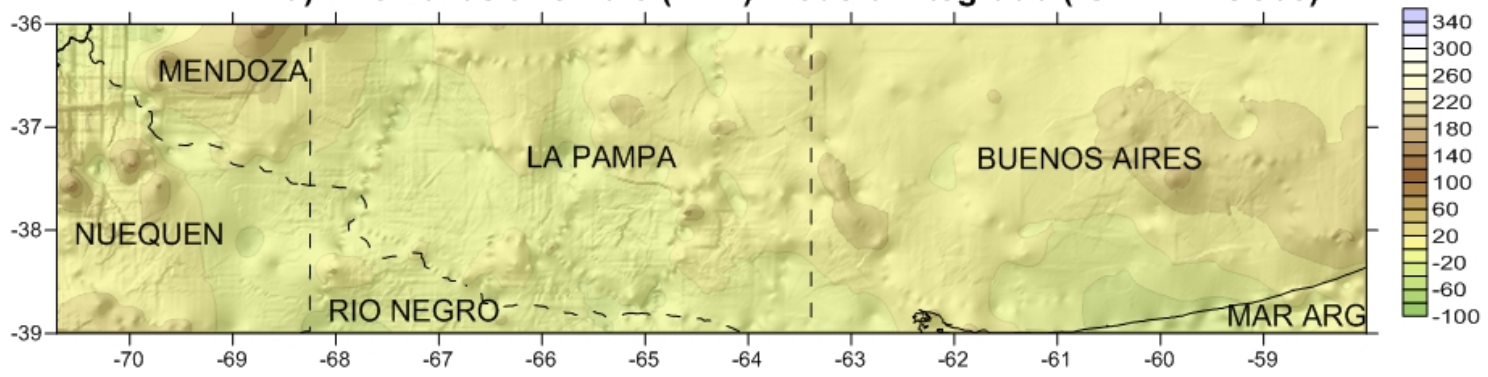

b) Anomalías aire libre (AAL) EGM2008

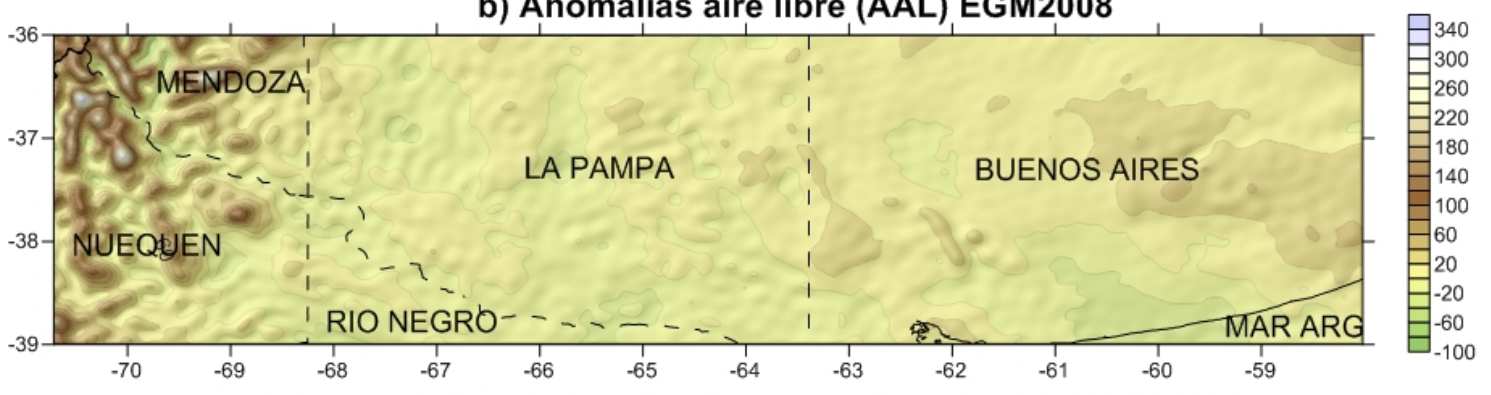

c)Diferencia AAL EGM2008 - Modelo integrado (IGN+DNSC08)

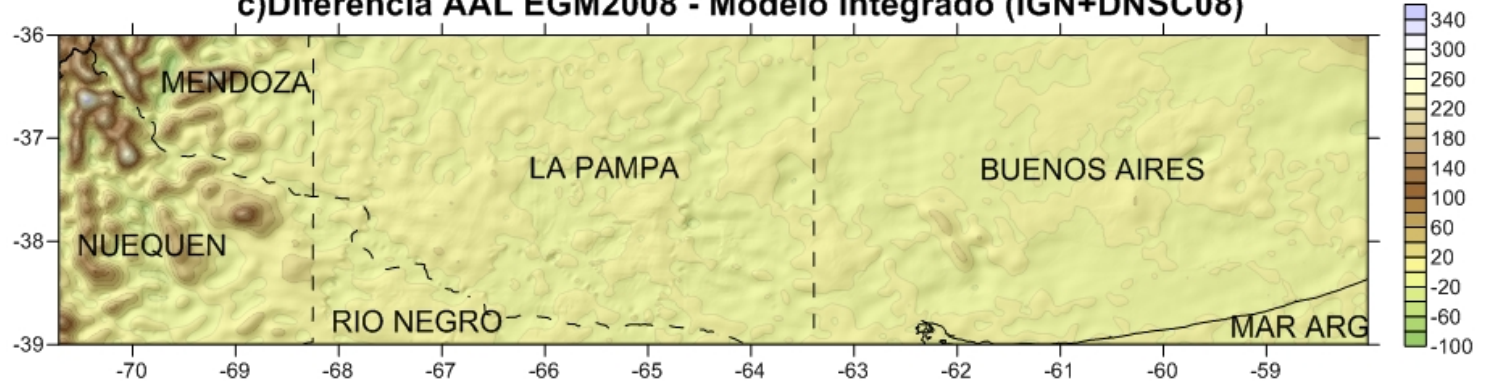

Figura 5.6.- Comparación entre anomalías de aire libre. a) Modelo integrado (IGN+DNSC08). b) EGM2008. c) Diferencias. Las AAL están expresadas en mGal 
Claramente las mayores diferencias están ubicadas en el sector oeste donde se ubican las mayores pendientes. En este sector se encuentran las diferencias de anomalías AAL más altas, hasta una magnitud de $336 \mathrm{mGal}$, donde también se ubican las mayores diferencias entre alturas IGN-DTM 2006 (755 m, ver en Tabla 5.1). Este comportamiento es una consecuencia de la fuerte correlación positiva existente entre las AAL y la topografía.

\subsection{Anomalía de Bouguer del modelo geopotencial EGM2008 y su comparación con las determinadas con datos IGN}

Se obtuvieron las anomalías de Bouguer (AB) del modelo geopotencial global en la región de estudio, en una grilla regular con resolución de un minuto, resolución similar a la grilla obtenida en el Capítulo IV a partir de datos terrestres IGN.

El calculador del ICGEM proporciona AB plana simple, y utiliza una densidad de 2.670 $\mathrm{g} / \mathrm{cm}^{3}$ para la masa topográfica (alturas positivas) y una densidad de $(2.670-1.025) \mathrm{g} / \mathrm{cm}^{3}$ para las zonas de mar (alturas negativas). El modelo digital de elevaciones usado para las reducciones es el DTM2006.

El resumen estadístico de los valores AB de ambos modelos, se muestra en la Tabla 5.4.

Tabla 5.4.-Anomalías de Bouguer obtenidas a partir de datos IGN, del modelo EGM2008 y sus diferencias.

\begin{tabular}{l|l|l|l}
\hline & EGM2008 & IGN & Diferencia \\
\hline Num. Nodos Grilla & 138103 & 138103 & 138103 \\
\hline Valor Máximo de AAL [mGal] & 58.43 & 32.14 & 74.00 \\
\hline Valor Mínimo de AAL[mGal] & -234.12 & -183.07 & -82.52 \\
\hline Promedio [mGal] & -33.73 & -32.48 & -1.25 \\
\hline Desviación estándar [mGal] & \pm 40.96 & \pm 40.04 & \pm 8.58 \\
\hline
\end{tabular}

El rango de valores AB EGM2008 es de $292 \mathrm{mGal}$, es superior al rango de AB del modelo calculado con datos IGN que es de $215 \mathrm{mGal}$. Asimismo el rango de variación de las diferencias entre las $\mathrm{AB}$ es de $156 \mathrm{mGal}$, casi tres veces menor en magnitud que las diferencias en las AAL (418 mGal), lo que muestra que las AB presentan un comportamiento más suave y menos afectado por la topografía.

Para analizar el comportamiento de las AB para ambos modelos y sus variaciones, se realizó la diferencia entre ellos mediante la ecuación (5.5):

$$
\text { Dif . } A B=A B_{E G M 2008}-A B_{I G N} .
$$

El resultado de esta diferencia es otra grilla con la misma resolución de los dos modelos anteriores y cuya variable espacial es la diferencia de $\mathrm{AB}$ entre ambos. El resumen estadístico 
de las diferencias se muestra en la Tabla 5.4. El rango de las diferencia de AB entre ambos modelos es de $156.50 \mathrm{mGal}$, sin embargo el 83 \% de los puntos tienen una diferencia menor de $\pm 10 \mathrm{mGal}$ (ver Figura 5.7).

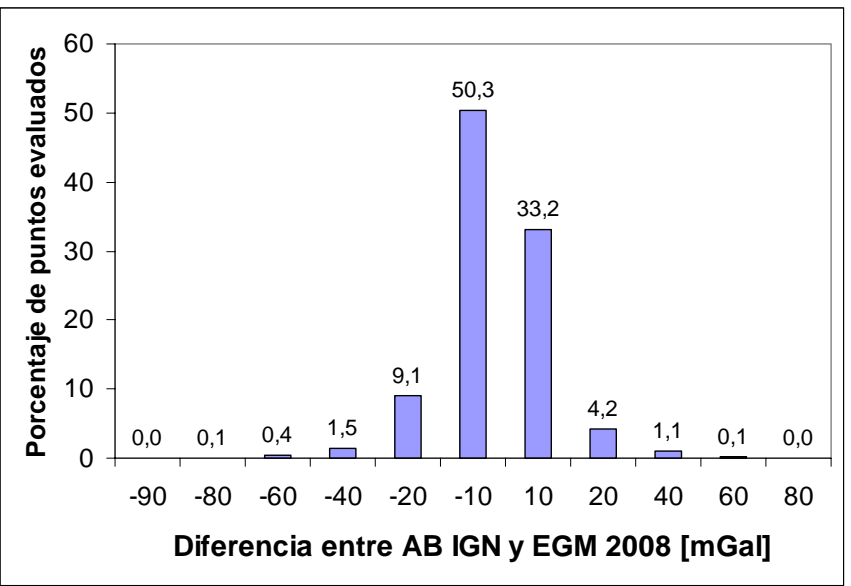

Figura 5.7.- Evaluación de diferencias de anomalías de Bouguer plana simple en nodos de grilla entre modelo calculado y EGM2008

Para visualizar la estructura espacial de $A B$ de ambos modelos y sus diferencias se confeccionaron los mapas de anomalías, que se muestran en la Figura 5.8. Claramente se advierte que las mayores diferencias tanto positivas como negativas se ubican en el sector Oeste.

\subsection{Conclusiones del capítulo}

Los modelos geopotenciales proveen las características regionales, o de larga longitud de onda, del campo de gravedad terrestre. Esto se advierte en la comparación de los mapas de anomalías gravimétricas del modelo EGM2008 con el obtenido a partir de datos terrestres, el primero representa de manera más suave las variaciones espaciales de las anomalías.

Con propósito de evaluar las anomalías de aire libre y Bouguer obtenidas en el Capítulo IV se comparan con las homónimas del modelo global. De la comparación se advierte un razonable ajuste, el $94 \%$ de los puntos evaluados (nodos de grilla) presentan diferencias de AAL menores que +/- 30mGal y el $83 \%$ presentan diferencias de AB menores que +/- $10 \mathrm{mGal}$. Esto se corresponde con las diferencias determinadas entre las alturas IGN y las alturas del modelo DTM2006, ya que para el 97\% de los puntos evaluados la diferencia de altura es menor que $100 \mathrm{~m}$ lo que produciría un error de $31 \mathrm{mGal}$. 
De la comparación entre las alturas IGN y las del modelo SRTM30 PLUS, usado en el cálculo de la corrección topográfica se advierte que para alturas menores a $500 \mathrm{~m}$ las diferencias entre ambas alturas es menor de $100 \mathrm{~m}$. Sin embargo, en las zonas de mayores pendientes las diferencias pueden tomar valores hasta $800 \mathrm{~m}$, donde también claramente se obtienen las mayores magnitudes de corrección topográfica calculada para obtener la anomalía de Bouguer completa.

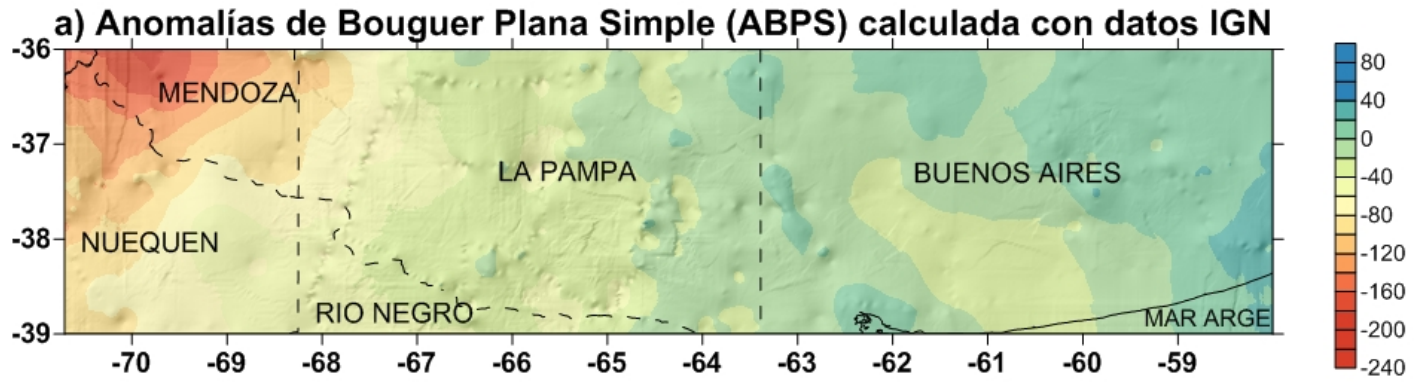

b) Anomalías de Bouguer Plana Simple (ABPS) EGM2008
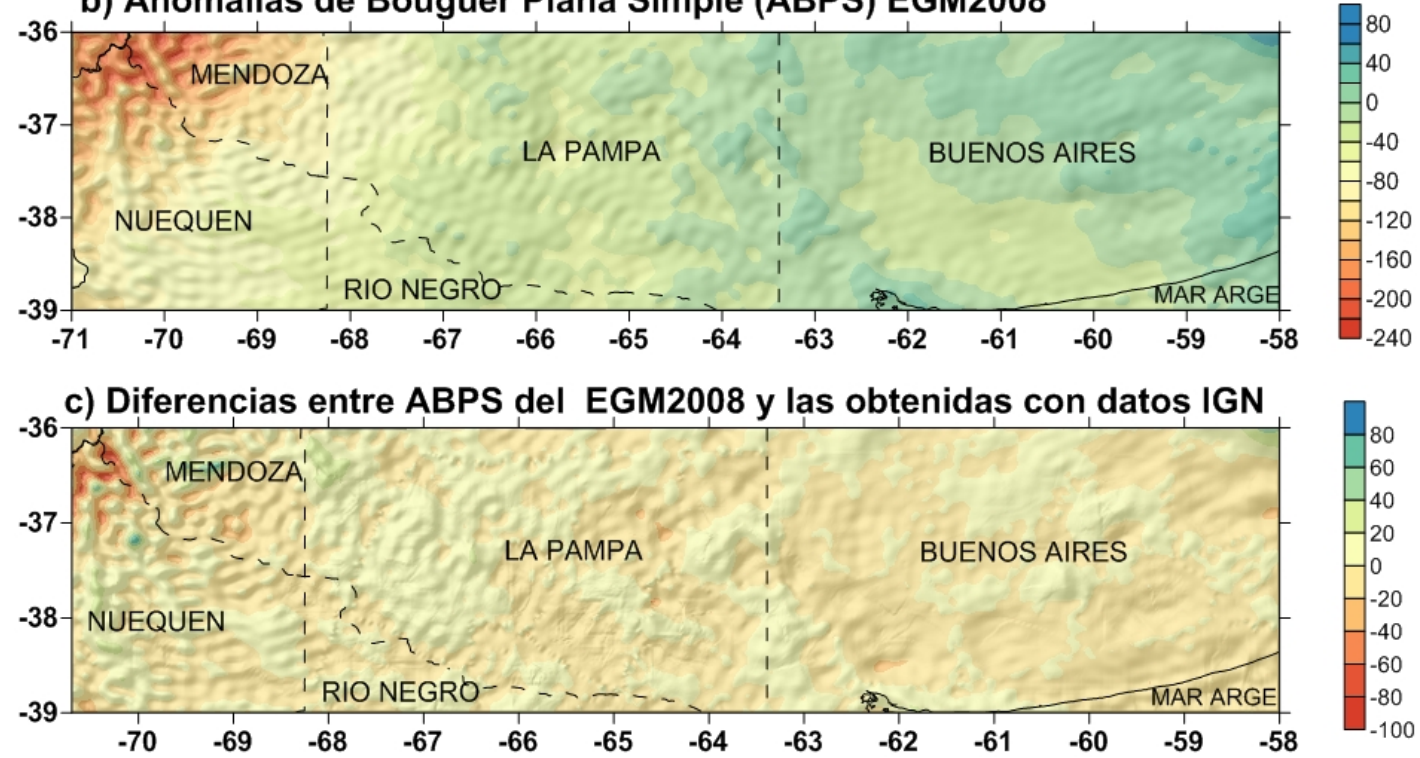

Figura 5.8.- Comparación entre anomalías de Bouguer plana simple. a) Obtenidas a partir de datos IGN. b) EGM2008. c) Diferencia. Las AB están expresadas en mGal 


\section{Capítulo VI}

\section{Isostasia y equilibrio isostático}

En este capítulo se analiza a priori el estado isostático de las estructuras geológicas a partir del análisis de anomalías de aire libre y de Bouguer y su correlación con la topografía. La isostasia estudia el equilibrio de la corteza terrestre. Si una estructura geológica está en equilibrio isostático las anomalías de aire libre son aproximadamente iguales a cero lejos de los bordes de la estructura, mientras que las anomalías de Bouguer están correlacionadas con la imagen especular de la topografía. De esta manera los mapas gravimétricos de aire libre y de Bouguer revelan a priori si una estructura está compensada o no.

\subsection{Desarrollo histórico}

Se podría pensar que las masas topográficas están sencillamente superpuestas sobre una corteza esencialmente homogénea. Si este fuera el caso, la reducción de Bouguer eliminaría las principales irregularidades del campo gravitacional, de modo que las anomalías de Bouguer serían muy pequeñas y fluctuarían aleatoriamente alrededor de cero. No obstante, sucede justamente lo contrario. Las anomalías de Bouguer en áreas montañosas son sistemáticamente negativas y pueden alcanzar grandes magnitudes, aumentando en promedio $100 \mathrm{mGal}$ por cada 1000 metros de altura (Heiskanen y Moritz., 1967). La única explicación posible es que existe algún tipo de deficiencia de masa bajo las montañas. Esto significa que las masas topográficas están compensadas de alguna manera.

Las variaciones a gran escala de las anomalías gravimétricas se deben a variaciones en la densidad y el grosor de la corteza, por ejemplo, la corteza oceánica es más densa y delgada que la corteza continental.

Los efectos de la isostasia se observaron por primera vez cuando en un levantamiento geodésico en el Norte de la India donde se encontró una discrepancia entre la distancia entre Kalianpur y Kaliana, medida por triangulación y medida astronómicamente, (Figura 6.1). La diferencia fue de aproximadamente $240 \mathrm{~m}$ (correspondiente a 5 segundos de arco) sobre una distancia de aproximadamente $600 \mathrm{~km}$ (Dobrin y Savit, 1988). La discrepancia se debe a la desviación horizontal de la plomada utilizada para definir la vertical en las mediciones astronómicas con la desviación causada por la atracción hacia ella de la masa presente en los Himalayas. J. H. Pratt intentado modelar la desviación usando la distancia conocida y la masa estimada del Himalaya encontró que sus resultados indicaban que la diferencia debería ser de 15 segundos de arco en lugar de 5 segundos de arco (Dobrin y Savit, 1988). 


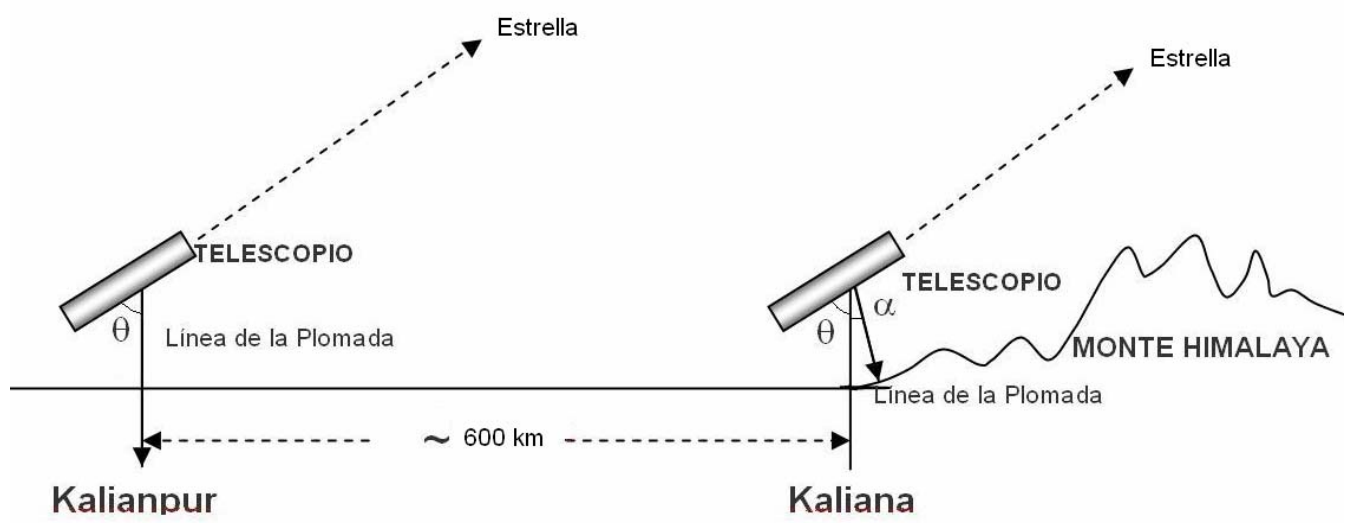

Figura 6.1. Primera observación de la isostasia.

Con el fin de explicar los resultados, George B. Airy sugirió que la corteza terrestre sería una estructura rígida que flota en un líquido más denso (Dobrin y Savit, 1988). Las porciones más gruesas de la corteza (por ejemplo montañas) flotan más alto fuera del agua y tienen raíces más profundas. El material menos denso en las raíces de la cordillera del Himalaya podría reducir la desviación de la vertical esperada de la línea de la plomada.

Cuatro años más tarde, Pratt propone una hipótesis alternativa en la que la corteza tiene una profundidad uniforme pero está compuesta de material de densidad variable. Toda la base de la corteza soporta un peso uniforme por unidad de área. Las montañas están compuestas de material menos denso que explica que la desviación de la línea de la plomada sea menor de lo previsto (Dobrin y Savit, 1988).

Las dos explicaciones son conocidas como los modelos de isostasia local de Airy y Pratt, respectivamente. La isostasia estudia el equilibrio de la corteza terrestre. Los modelos de isostasia asumen que hay una cierta profundidad llamada profundidad de compensación en la que las presiones ejercidas por las columnas de rocas son iguales. La presión litostática ejercida por un bloque de corteza está dada por:

$$
P=\rho g h
$$

\subsection{Base matemática de la hipótesis de Airy}

Observando la Figura 6.2 (Fowler, 2005), de acuerdo la hipótesis de Airy, en una profundidad de compensación arbitraria, que generalmente es la profundidad de la raíz más profunda de la corteza, se cumple la condición de la ecuación (6.2):

$$
\begin{aligned}
t \rho_{u} & +r_{1} \rho_{s}=\left(h_{1}+t+r_{1}\right) \rho_{u} \\
& =\left(h_{2}+t+r_{2}\right) \rho_{u}+\left(r_{1}-r_{2}\right) \rho_{s}
\end{aligned}
$$




$$
=d \rho_{w}+\left(t-d-r_{3}\right) \rho_{u}+\left(r_{1}+r_{3}\right) \rho_{s} .
$$

donde $\rho_{\mathrm{u}}$ es la densidad de la capa superior, $\rho_{\mathrm{s}}$ es la densidad un sustrato, $\rho_{\mathrm{W}}$ es la densidad del agua y t es el espesor de la corteza regular que suele establecerse en un valor entre 30-40 km (Torge, 1989; Torge, 2001).

Una montaña de altura h por lo tanto tendría una raíz $\mathrm{r}_{1}$ dada por:

$$
r_{1}=\frac{h_{1} \rho_{u}}{\rho_{s}-\rho_{u}}
$$

Del mismo modo, una profundidad d por debajo del nivel del mar tendría una anti-raíz $\mathrm{r}_{3}$ dada por:

$$
r_{3}=\frac{d\left(\rho_{u}-\rho_{w}\right)}{\rho_{s}-\rho_{u}}
$$

Las ecuaciones (6.2) a (6.4) pueden escribirse en términos de densidad de la corteza $\rho_{\mathrm{c}} \mathrm{y}$ el manto $\rho_{\mathrm{m}}$ (Fowler, 2005):

$$
\begin{gathered}
r_{1}=\frac{h_{1} \rho_{c}}{\rho_{m}-\rho_{c}} \\
r_{3}=\frac{d\left(\rho_{c}-\rho_{w}\right)}{\rho_{m}-\rho_{c}} .
\end{gathered}
$$

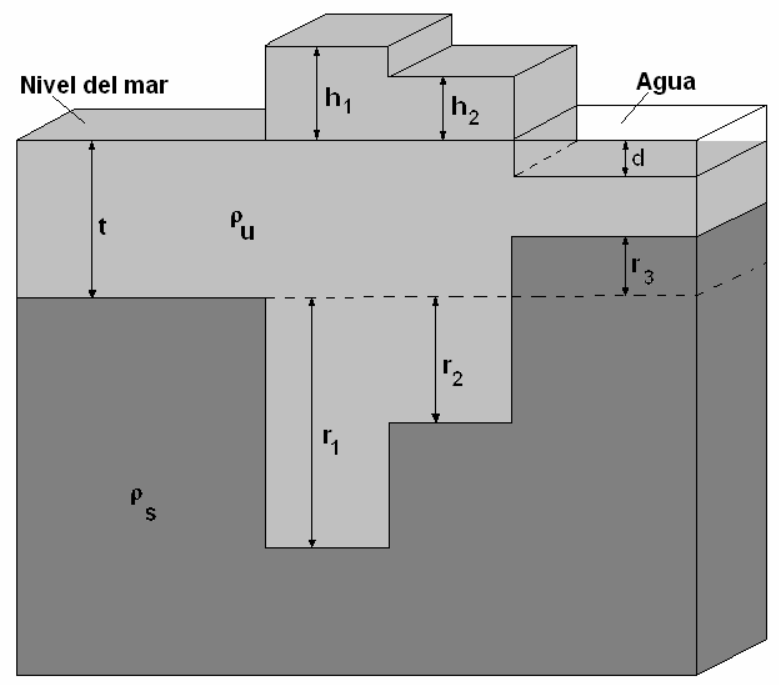

Figura 6.2. Geometría del modelo de Airy (Fowler, 2005). 


\subsection{Base matemática de la hipótesis de Pratt}

En el modelo de Pratt, la profundidad de compensación es constante. Tomando la base de la capa superior como la profundidad de compensación, tenemos:

$$
\begin{aligned}
\rho_{u} D & =\left(h_{1}+D\right) \rho_{1} \\
& =\left(h_{2}+D\right) \rho_{2} \\
& =d \rho_{w}+(D-d) \rho_{d} .
\end{aligned}
$$

Siendo D el espesor de la litosfera que en general se establece en 100 a 120 km (Torge, 1989;

Torge, 2001).

La compensación se logra para una montaña cuando:

$$
\rho_{1}=\rho_{u}\left(\frac{D}{h_{1}+D}\right) .
$$

Los océanos están sustentados por materiales de mayor densidad:

$$
\rho_{d}=\frac{\rho_{u} D-\rho_{W} d}{D-d}
$$

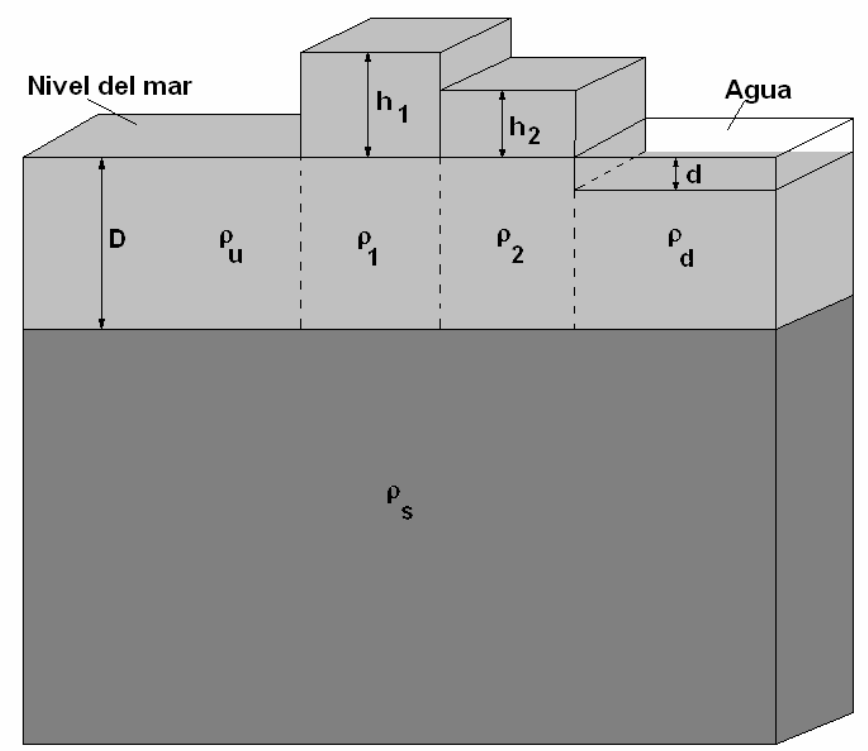

Figura 6.3. Geometría del modelo de Pratt (Fowler, 2005).

\subsection{Equilibrio isostático}

La presión total ejercida por cada columna vertical dividida por la aceleración de la gravedad debe ser constante: 


$$
\frac{P}{g}=\rho_{a} h_{a}+\rho_{w} h_{w}+\rho_{c} h_{c}+\rho_{m} h_{m}=C_{1}
$$

y el espesor total de cada columna vertical es constante

$$
T=h_{a}+h_{w}+h_{c}+h_{m}=C_{2} .
$$

el índice a se refiere a la atmósfera (hasta el nivel más alto de la topografía), w se refiere al agua, c se refiere a la corteza, y m se refiere al manto. Si la columna isostática se puede determinar (o suponerse) para un área, entonces la solución de ambas ecuaciones con las correspondientes restricciones adicionales pueden permitir la determinación del espesor o densidad por debajo de otras áreas.

En el caso de equilibrio isostático en la profundidad de compensación, se deben cumplir dos condiciones: la igualdad de presión, establecida en la ecuación (6.10), y la igualad de espesores expresada en la ecuación (6.11).

\subsection{Determinación del equilibrio isostático}

Una manera sencilla de tener una primera visión de si una estructura geológica se encuentra en equilibrio isostático es examinar las anomalías de aire libre y de Bouguer (Fowler, 2005). Si una cadena montañosa está en equilibrio isostático las anomalías de aire libre son aproximadamente iguales a cero sobre la estructura y las anomalías de Bouguer serán claramente negativas. Las anomalías de Bouguer iguales a cero son evidencia de una estructura no compensada. Así, los mapas de anomalías de aire libre y de Bouguer revelan parcialmente si una estructura está compensada o no. Un análisis más riguroso requiere naturalmente de un conocimiento lo más detallado posible de la estructura cortical correspondiente.

Sin embargo, para una señal de gravedad de corta longitud de onda $(<100 \mathrm{~km})$, las anomalías de aire libre son proporcionales a la altura $\mathrm{H}$ y las anomalías de Bouguer son aproximadamente cero, ya que la corta longitud de onda de la gravedad no esta compensada, debido a presencia de la litosfera (ver los mapas de AAL y AB en las Figuras 4.15 y 4.16, respectivamente). En este punto, es útil volver a examinar las anomalías de aire libre y de Bouguer obtenidas en el capítulo anterior.

Para las mediciones de la superficie del mar la anomalía de aire libre será cero cuando la superficie del mar se corresponda con el geoide. La corrección de Bouguer se hace para compensar la menor densidad del agua (en relación a la corteza) entre el fondo marino y la superficie del mar. Se utiliza una diferencia de cota igual a la profundidad del océano y por lo general una densidad de $1.640 \mathrm{~g} / \mathrm{cm}^{3}$. 
Debido al gran cambio en la anomalía de Bouguer que ocurre en un margen continental pasivo como se observa en la Figura 6.4, generalmente los geofísicos utilizan la anomalía de Bouguer para los datos terrestres y la anomalía de aire libre para datos marinos (Lowrie, 2007).
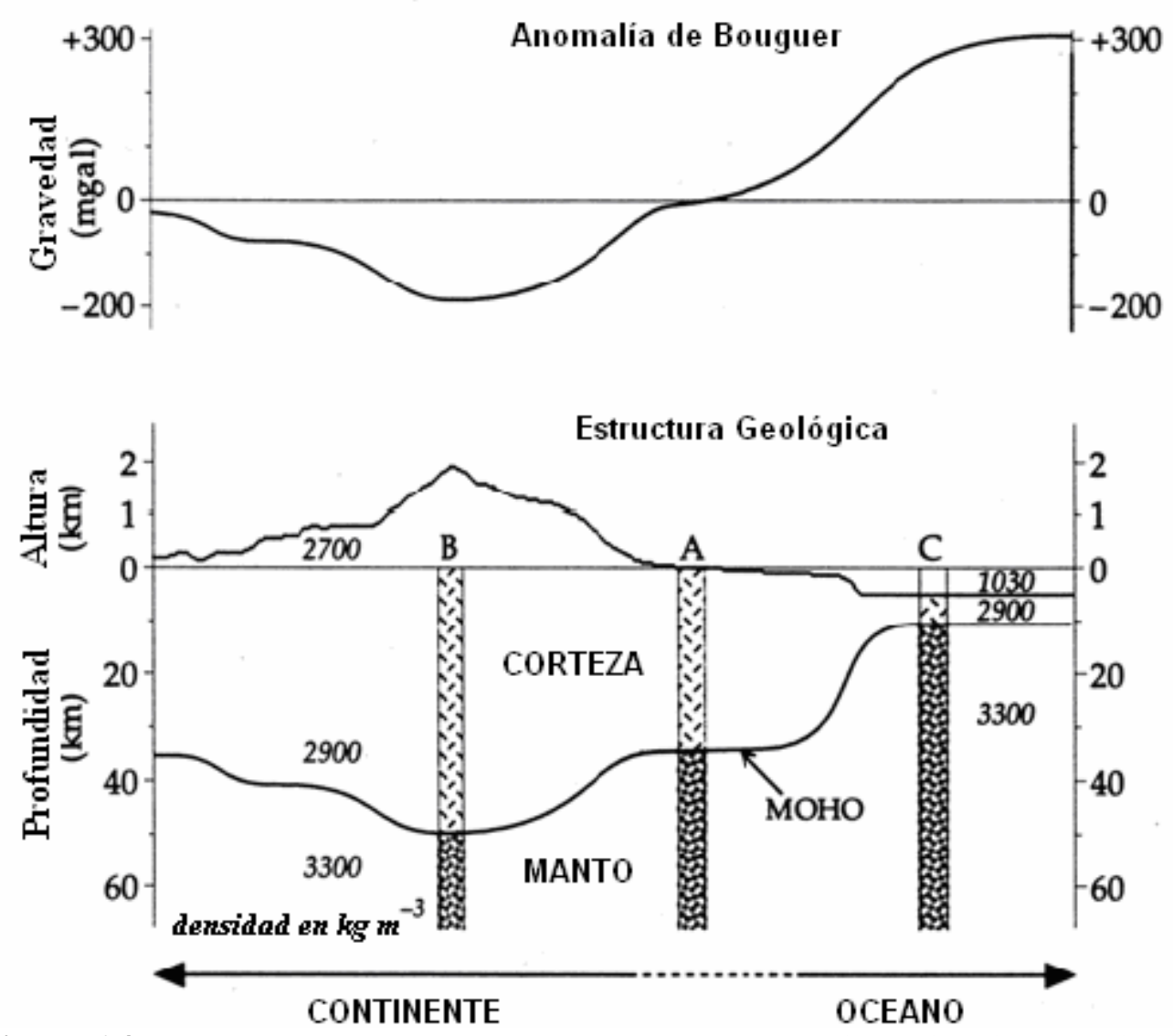

Figura 6.4. Anomalía de Bouguer a través de un margen continental pasiva (Fowler, 2005).

\subsection{Análisis cualitativo de los mapas de anomalías de aire libre y anomalías de Bouguer}

Con el fin de evaluar cuantitativamente las anomalías de gravedad se clasifican los valores de anomalías de aire libre (AAL) y de Bouguer plana completa (AB) sobre nodos de una grilla (Figura 6.5), con resolución de un minuto de arco ( 2 km en el Ecuador).

Los valores de AAL para toda la zona de trabajo presentan una variación que oscila entre máximos de $+80 \mathrm{mGal}$ ubicados en la provincia de Neuquén y mínimos de -38 mGal en la plataforma del mar Argentino, en la zona de la cuenca de Claromecó.

En la Figura 6.5.a. se muestra la variación de AAL. Se advierte que el 54 \% de los puntos toman valores de AAL positivos. El mayor porcentaje de puntos (40\%) presentan valores entre +5 y +20 mGal, y se ubican en gran parte de las provincias de Buenos Aires y La Pampa, y parte de las provincias de Mendoza y Neuquén, correlacionados con topografía baja (Figura 6.6). El 46 \% de los puntos asumen valores negativos y se ubican en parte de las provincias de 
La Pampa, Río Negro y Este de Neuquén, también al Sur de Buenos Aires en la cuenca de Claromecó. Del total de puntos analizados sólo el 21\% asumen valores de AAL en un entorno de $\pm 5 \mathrm{mGal}$. Estos valores bajos cercanos a cero, se corresponden con sectores que bordean Los Andes al Oeste y la cuenca de Claromecó al Este (Figuras 6.5 y 6.7). Los picos positivos de AAL se encuentran en sector Sur de Mendoza y Nor-Oeste de Neuquén. También se advierte otro pico positivo de AAL (60 mGal) en el sector Este, en el sistema de Tandil, provincia de Buenos Aires.

En la Figura 6.5.b. se muestra la variación de $A B$. Los valores de $A B$ presentan una variación que oscila entre máximos de $+50 \mathrm{mGal}$ al Este de la provincia de Buenos Aires y mínimos de alrededor de -180 mGal al Sur de la provincia de Mendoza y Norte de Neuquén. Sólo el 20\% de los puntos presentan valores positivos y se ubican en el sector Este, en la zona de la cuenca sedimentaria del Salado y del sistema de Tandil (Buenos Aires), (Figura 6.5 y 6.6). El resto de los puntos analizados (80\%) toman valores negativos, que disminuyen gradualmente hacia el Oeste, con mayores magnitudes en el sistema de Los Andes.

Para analizar las anomalías gravimétricas en relación con la topografía se realizó un trazado de isolíneas de AAL y AB sobre un mapa topográfico (Figura 6.6). En la misma se advierte que los picos positivos de AAL ubicados al Oeste, corresponden al Bordo Alto del Payún y Sierras de Chachauen, (provincia de Mendoza), con un pico de altura correspondiente al cerro Payún de 3680 m, y las Sierra de Cochico y Cordillera del Viento (provincia de Neuquén), con un pico de altura correspondiente al volcán Tromen de 3980 m de altura. En este sector también se observan valores mínimos de AB, en un rango entre -140 a -180 mGal lo que pone de manifiesto el efecto de las raíces isostáticas que soportan a la cordillera de los Andes.

El otro pico positivo de AAL, ya mencionado, se ubica en el sistema de Tandil, las alturas de este sistema no superan los $500 \mathrm{~m}$. En este sector las AB toman valores también positivos y cercanos a cero. Estos valores de AB predominantemente positivos indican que los modelos isostáticos clásicos no podrán explicar a priori este comportamiento (Del Cogliano, 2006). Se infiere a priori que no existe equilibrio isostático.

Al Oeste del sistema de Tandil se encuentran las sierras de la Ventana, con alturas que llegan a los 1239 m (Cerro Tres Picos), (Figura 6.6). En este sector se advierten AB negativas y AAL positivas y cercanas a cero, lo que mostraría una estructura compensada.

Al Norte del sistema de Tandil se encuentra la cuenca del Salado. En este sector las AAL son positivas y toman valores entre $+5 \mathrm{y}+20 \mathrm{mGal}$, mientras que las $\mathrm{AB}$ también asumen valores positivos que crecen hacia el Este hasta valores de +20 mGal. Las cuencas sedimentarias presentan muchas veces cortezas atenuadas y de allí los valores de anomalías de Bouguer positivas como las que se observan hacia el Este. Podría inferirse a priori un desequilibrio isostático. 
Los valores mínimos de AAL se ubican en la cuenca de Claromecó al Este (Buenos Aires), y en la confluencia de ríos Limay y Neuquén al Oeste (Río Negro). En ambos sectores las $\mathrm{AB}$ son negativas con valores medios de $-30 \mathrm{mGal}$ y $-50 \mathrm{mGal}$, respectivamente. La cuenca de Claromecó es conocida también como cuenca interserrana bonaerense o cuenca paleozoica de la provincia de Buenos Aires (Introcaso y Ruiz, 2005). Si bien para este tipo de cuencas sedimentarias se esperan valores negativos de las AAL, por sus magnitudes no se puede a priori asegurar su estado de equilibrio.

En el sector central de la zona de estudio, en las provincias de La Pampa y Río Negro, las AAL son bajas, con valores entre -20 a $+20 \mathrm{mGal}$. En tanto que las AB toman valores entre -5 a -60 mGal. Valores de AAL bajos y AB correlacionadas especularmente con la topografía mostrarían una estructura compensada.

En el extremo Sur-Este de la zona de estudio se encuentra el Mar Argentino, y como se expresó anteriormente, en presencia de mar, la situación es diferente. Las anomalías de Bouguer en el mar se calculan reemplazando el agua por material rocoso y por lo tanto el gran término positivo de la corrección de Bouguer enmascara las estructuras locales. Por lo tanto, convenimos el uso de mapas de anomalías de Bouguer en la tierra y los mapas de anomalías de aire libre en el mar. En la Figura 6.5 se observa la continuidad de la cuenca de Claromecó en el mar. La continuidad de la cuenca en el mar se advierte claramente en la Figura 4.14 del Capítulo IV.

El razonamiento realizado puede inferirse también a partir del análisis de la correlación espacial entre las AAL y las AB. Para ello se superpuso un trazado de isolíneas de AB sobre un mapa de AAL (Figura 6.7). La equidistancia entre curvas es de $20 \mathrm{mGal}$. Se advierten hacia el Oeste los picos positivos de AAL y mínimos negativos de $\mathrm{AB}$ en correspondencia con Los Andes. Asimismo se observa al Este la estructura de Tandil con valores positivos altos de AAL y positivos de AB. También se advierte la curva de AB que encierra la cuenca de Claromecó con valores negativos de AAL. 


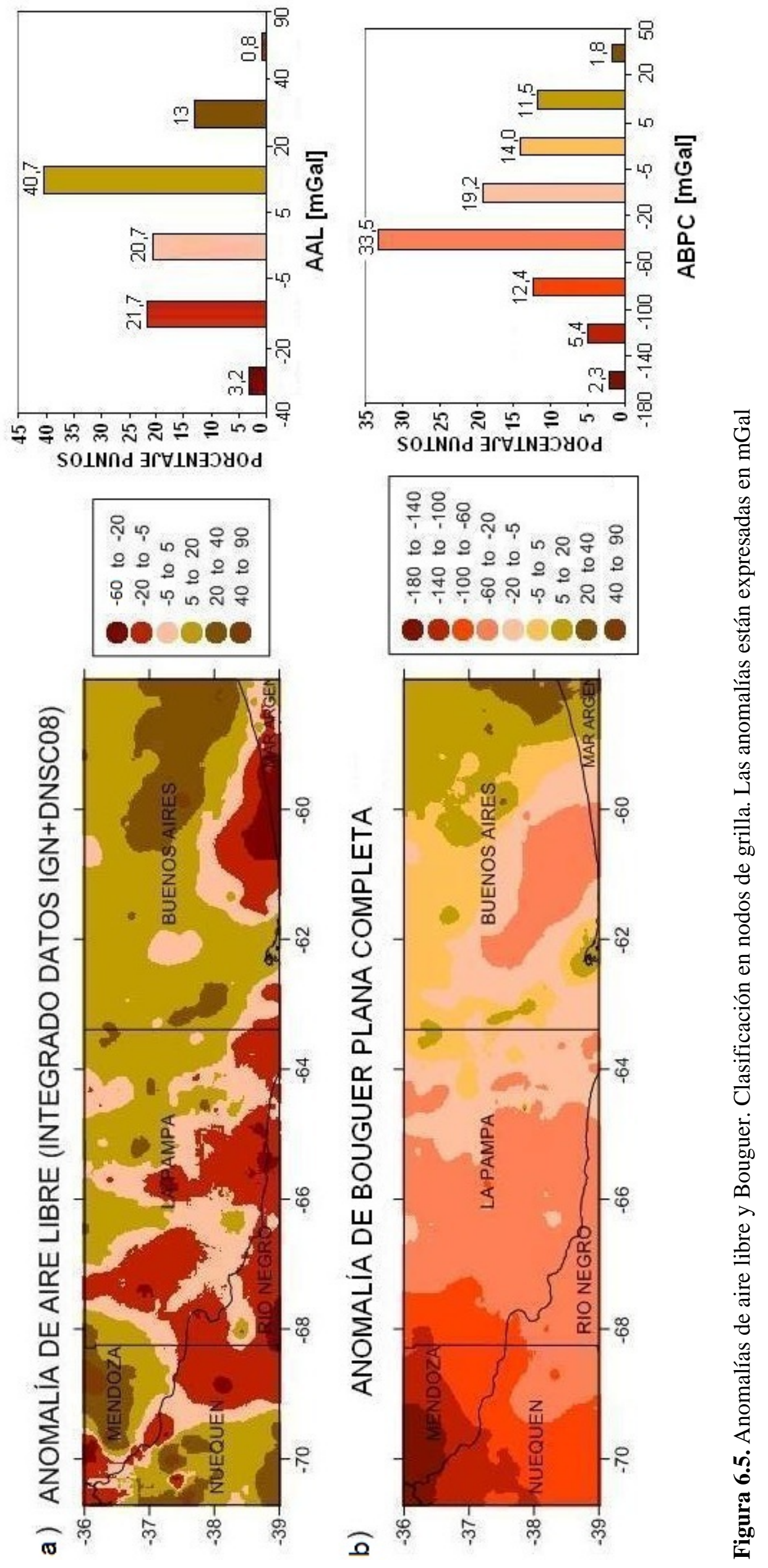



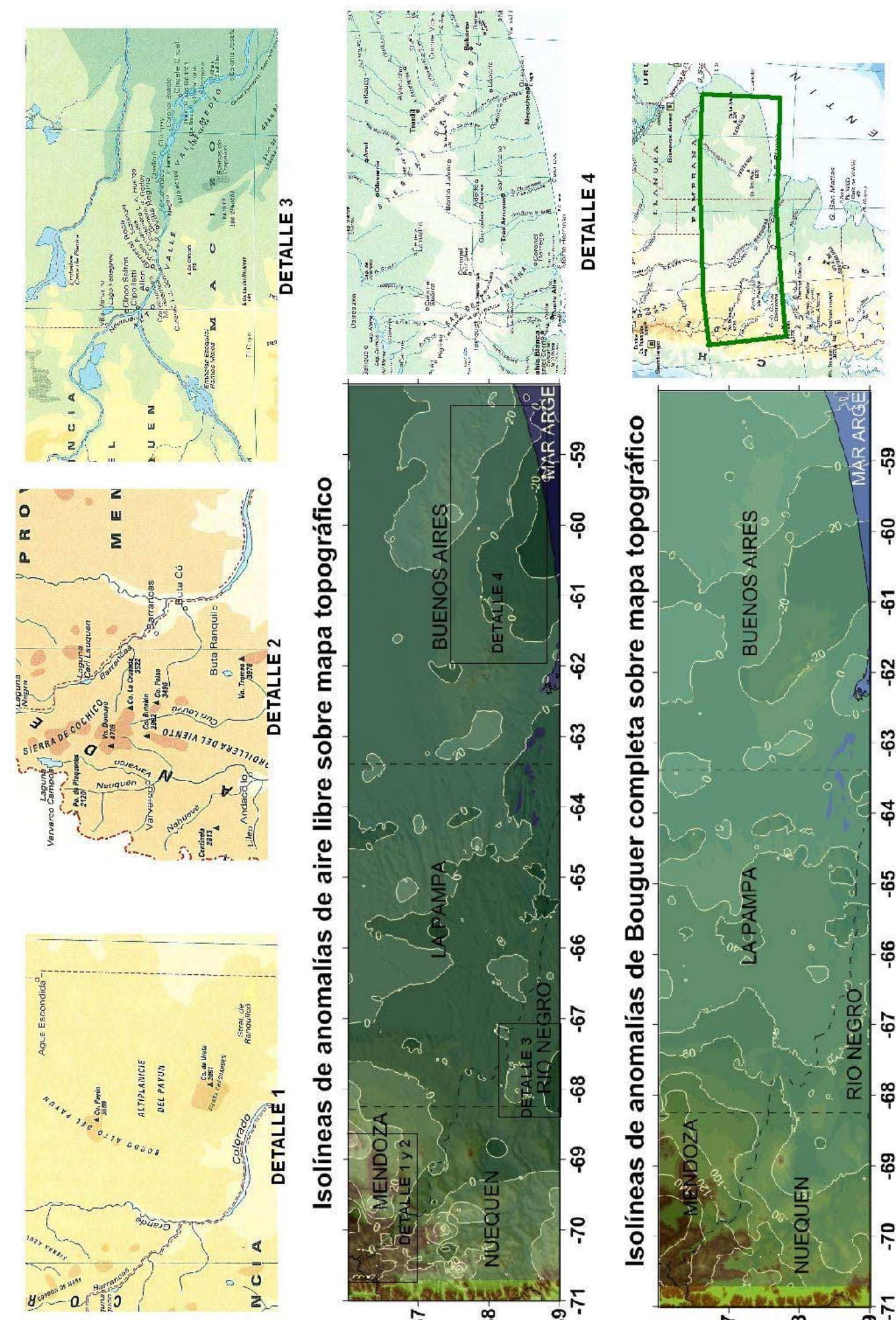

ธฺุ

을
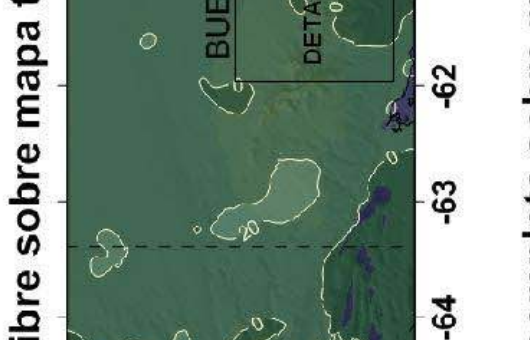

\%
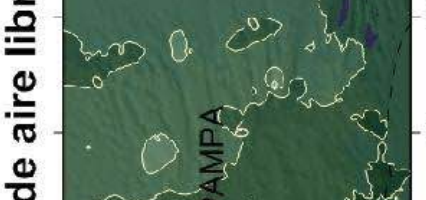

ఫ़
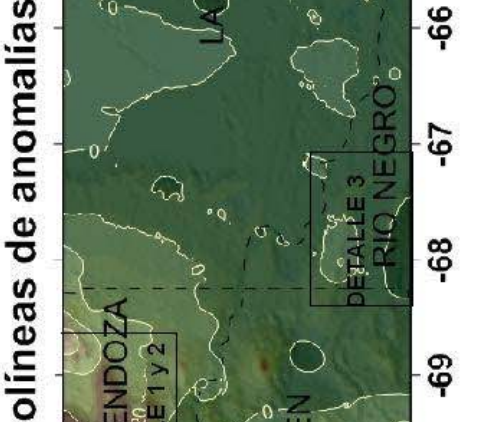


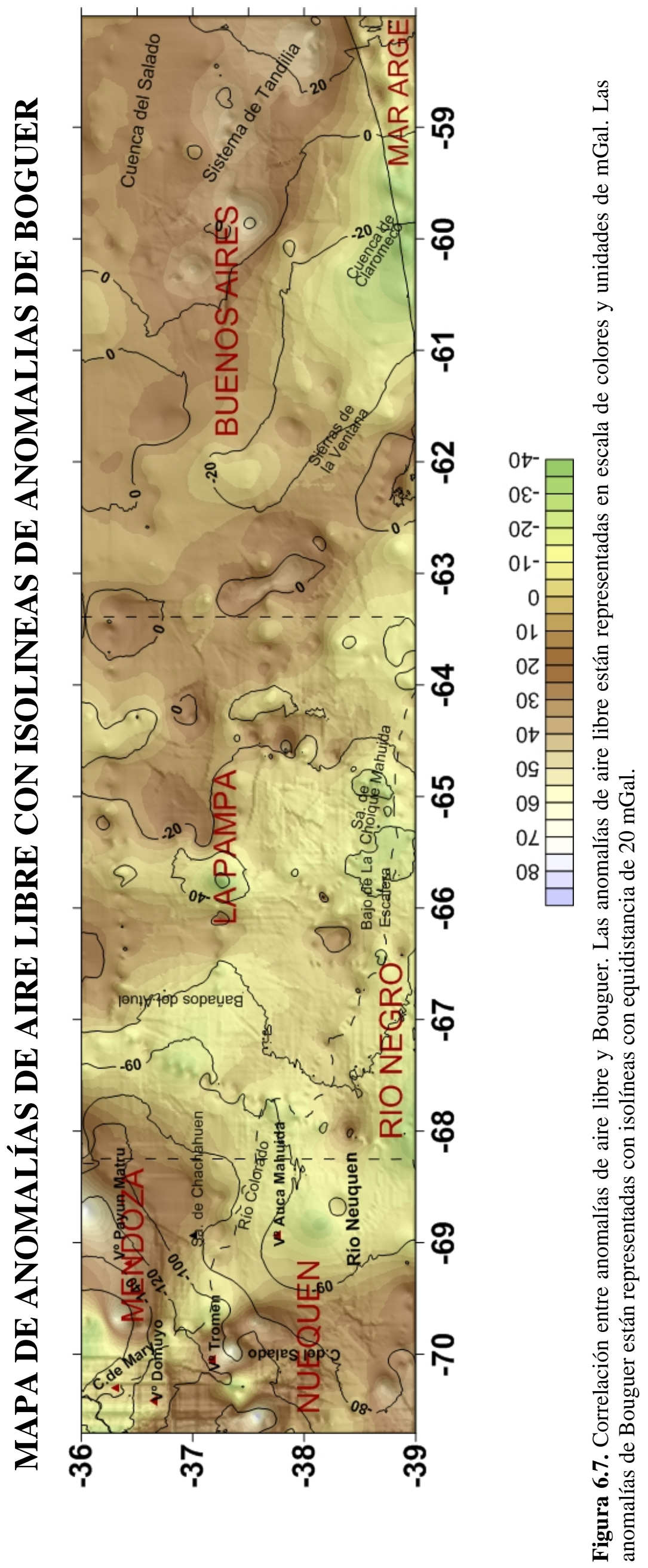


6.7 Análisis de anomalías de aire libre y Bouguer en relación con la topografía sobre perfiles

Las variaciones de AAL y AB se analizan en relación con las alturas, sobre perfiles topográficos y de anomalías gravimétricas a lo largo de los paralelos (Figura 6.8).

PARALELO $-36^{\circ}$
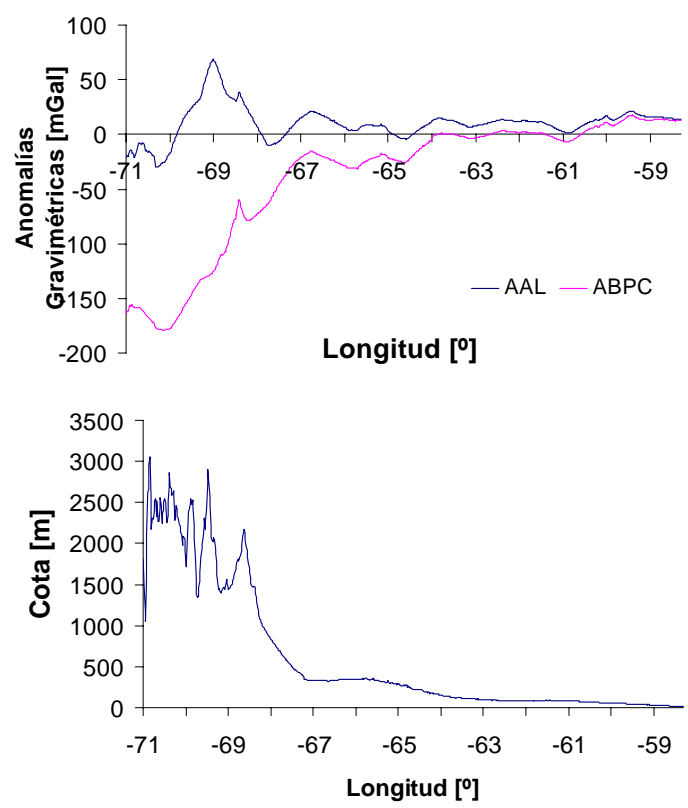

(a)
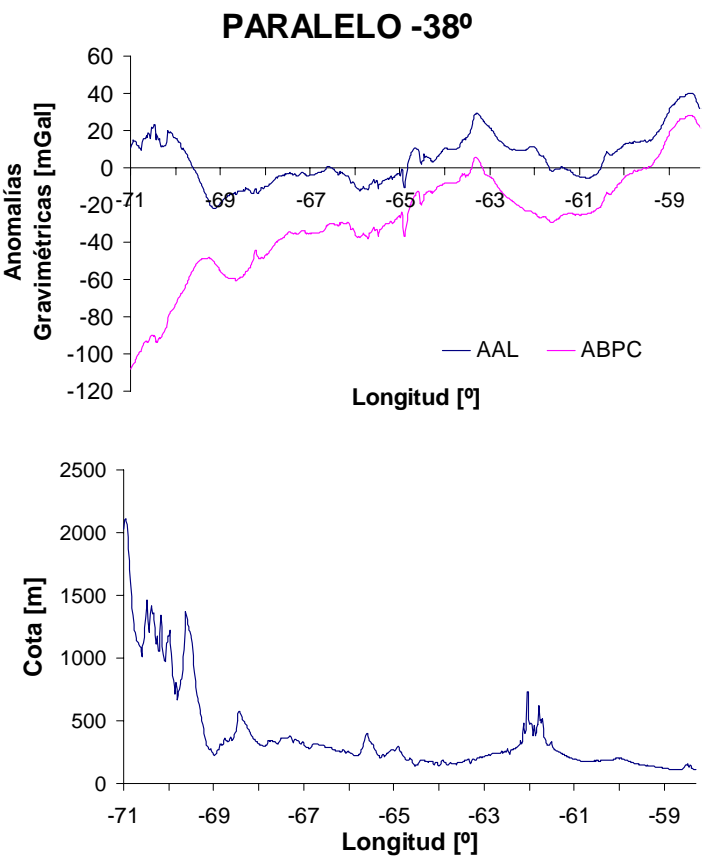

(c)
PARALELO $-37^{\circ}$
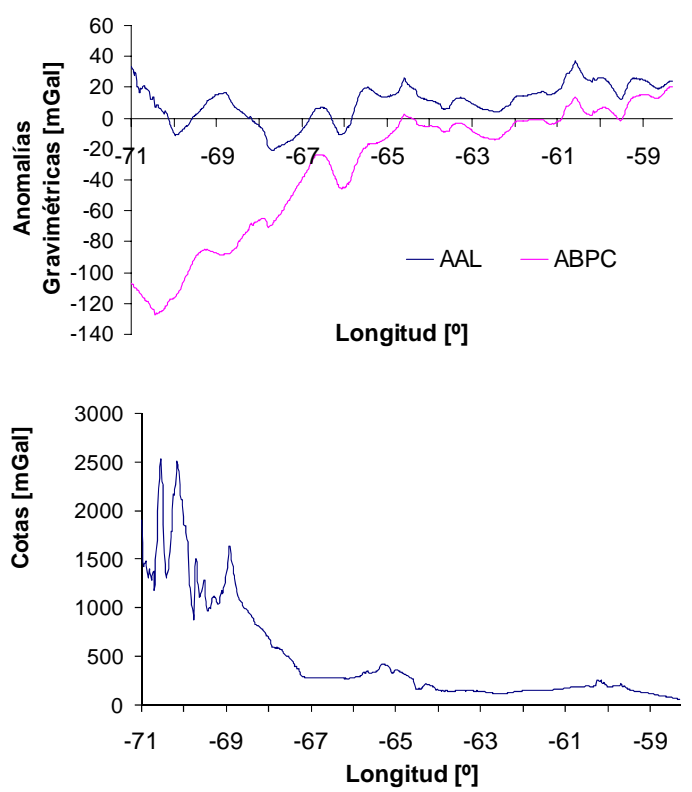

(b)
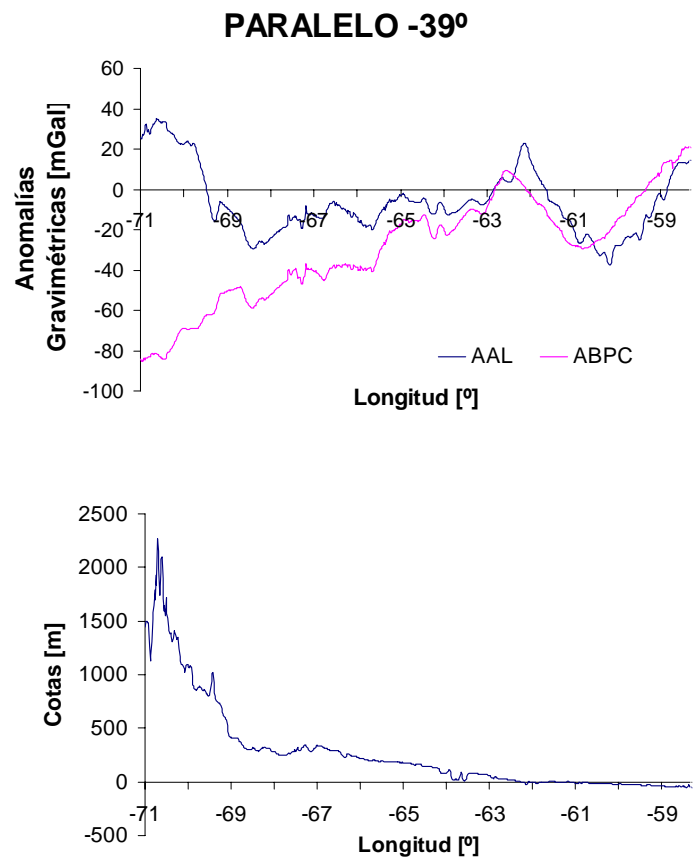

(d)

Figura 6.8. Perfiles de anomalías gravimétricas de aire libre y Bouguer, y perfiles topográficos a lo largo de paralelos. 
Los perfiles se trazaron sobre los paralelos $36^{\circ}, 37^{\circ}, 38^{\circ}$ y $39^{\circ}$ de latitud Sur. En los cuatro perfiles (Figura 6.8) se advierte que hacia el Oeste y en correspondencia con las mayores alturas, los valores de $\mathrm{AB}$ son negativos y de gran magnitud, aunque hacia el sur observamos una disminución en la magnitud, resultados similares encontramos en Introcaso et al., (1996). Asimismo las AAL en general toman valores menores y cercanos a cero, en un rango de \pm 20 mGal.

En el paralelo $-36^{\circ}$ se observa un pico de valores positivos de AAL ( $>+50 \mathrm{mGal}$ ) en la longitud de $-69^{\circ}$, lo que se corresponde con el Bordo Alto del Payún (Mendoza). Hacia el sector central, entre los meridianos $-63^{\circ}$ y $-68^{\circ}$ la topografía es menos abrupta y se corresponde con anomalías de AAL bajas y positivas ( $<+20 \mathrm{mGal})$.

En el paralelo $-37^{\circ}$ (Figura 6.8.b) se advierte que los valores de AAL son predominantemente positivos y no superan los $+20 \mathrm{mGal}$, a excepción del sector comprendido entre los meridianos de $-67^{\circ}$ y $-68^{\circ}$, donde valores negativos de AAL se corresponden con los Bañados del Atuel. Se observa un pico de AAL de $+37 \mathrm{mGal}$ que corresponde al extremo Norte del sistema de Tandilia.

A partir del paralelo $-38^{\circ}$ ya se advierten valores de AAL bajos pero negativos (Figura 6.8.c. y d.). En todos los perfiles las AB son en general negativas. Hacia el sector Este, la topografía en general es bastante baja, con pequeñas elevaciones que se advierten en el paralelo $-38^{\circ}$ (Figura 6.8.c.), que muestra el sistema de la Ventana. En general las AAL son bajas y cercanas a cero, en tanto las AB también son bajas y toman valores positivos y negativos.

En particular en el paralelo $-38^{\circ}$, se observan valores altos de AAL al Este, que no se corresponden con las elevaciones, en este sector se ubica el sistema de Tandil, que ya se había inferido que no existiría equilibrio isostático, al menos sustentado en alguna de las teorías isostáticas expuestas. Sin embargo una visión geológica que considere la génesis del sistema puede explicar las anomalías positivas (Del Cogliano, 2006). Los procesos geológicos de formación de las sierras, estarían vinculadas a una colisión entre continentes ocurridos hace 1770 Ma que habrían producido montañas, actualmente erosionadas. Las anomalías gravimétricas, en principio podrían relacionarse con la zona de sutura entre ambos continentes, en la que el fondo oceánico y manto compondrían una cuña tectónica durante la colisión (Del Cogliano, 2006).

En el paralelo $-39^{\circ}$ (Figura 6.8.d.), y a partir de la longitud de $-62.30^{\circ}$ aproximadamente, comienza la zona de mar donde se advierten valores negativos de AAL, con un pico negativo de $-29.30 \mathrm{mGal}$ a una longitud de $-60.5^{\circ}$, en correspondencia con la cuenca de Claromecó. 


\subsection{Conclusiones del capítulo}

Las anomalías de aire libre y Bouguer no pueden revelarnos el mecanismo de compensación (Pratt o Airy) pero la correlación que existe entre dichas anomalías y la topografia nos brinda información que sirve para un análisis a priori del estado isostático de la estructura. Otra forma de determinar el estado de compensación isostática de una estructura, que no será abordado en esta Tesis es calculando las anomalías isostáticas.

Las anomalías isostáticas (AI) remueven las masas compensadas según algún modelo isostático como los de Airy y Pratt y se calculan mediante la siguiente fórmula:

$$
A I=g_{O b s}-\delta g_{T O P}+\delta g_{a t m}+\delta g_{h}+\delta g_{I}-\gamma
$$

donde $\delta \mathrm{g}_{\mathrm{I}}$ es el efecto gravimétrico de las masas compensadas según el modelo de compensación adoptado.

Si los modelos son exactos y existe equilibrio isostático, la anomalía isostática correspondiente debe ser nula o cercana a cero. Valores de anomalía isostática sistemáticamente por encima o por debajo de cero implican desviaciones del equilibrio de la corteza respecto del modelo empleado. Las masas superficiales deben tener tendencia a subir (si la anomalía es negativa) o a bajar (si es positiva).

El cálculo de las anomalías isostáticas es dificultoso porque hay que conocer el modelo isostático de compensación que rige en cada zona de estudio y que mejor ajuste con sus características geológicas por lo que es necesario disponer de información sísmica y tectónica del área a estudiar.

Del análisis cualitativo de las AAL y AB, se observa que en gran parte de la región (más del 80\%) los valores de AAL varían en un entorno de $\pm 20 \mathrm{mGal}$. En esta región los valores de $A B$ presentan una imagen especular de la topografía, esto se advierte más claramente en el análisis por perfiles. Estos valores infieren a priori estructuras compensadas a escala regional.

Valores positivos altos de AAL correlacionados con la topografía se muestran en el sector Oeste, a la altura del paralelo $-36^{\circ}$, que corresponden al Bordo Alto del Payún y Sierras de Chachauen, (provincia de Mendoza), con un pico de altura correspondiente al cerro Payún de 3680 m. Un comportamiento similar se advierte en las Sierra de Cochico y Cordillera del Viento (provincia de Neuquén). Estos valores mostrarían una estructura que no está en equilibrio isostático. Otro pico positivo de AAL se ubica al Este en el sistema de Tandil con valores de AB positivos, esta estructura no estaría compensada de acuerdo a los modelos isostáticos clásicos. 


\section{Capítulo VII \\ Ondulación del Geoide del Modelo Geopotencial EGM2008}

En este capítulo se analizan las ondulaciones del geoide obtenidas a partir del modelo geopotencial EGM2008 en toda la zona de estudio y se realizan filtrados matemáticos de los mismos mediante el truncamiento del desarrollo en serie para grado bajo, y restando su contribución al modelo de grado completo. Con la ondulación del geoide residual se logran detectar la presencia de estructuras geológicas. Finalmente se correlacionan las estructuras con los mapas de anomalías de aire libre y Bouguer obtenidas en el Capítulo IV para establecer $a$ priori el estado isostático de las mismas.

\subsection{Ondulación del geoide}

El geoide se define como la superficie equipotencial del campo de gravedad terrestre que mejor representa al nivel medio del mar libre de efectos de mareas, olas, corrientes oceánicas que se generan por la influencia de los vientos, de los cambios en la salinidad y temperatura del agua de mar y de los cambios de la presión relacionados directamente con la atmósfera terrestre, etc., (Tocho, 2006).

El geoide, definido a partir del nivel medio del mar, se prolonga por debajo de los continentes. Su superficie presenta alteraciones debido a distribuciones de masas anómalas en el interior de la Tierra y presencia de masas exteriores al geoide (topografía). El geoide cambia con el tiempo por variaciones en la distribución de masa en el núcleo, en la litosfera por desprendimientos de hielos en los polos, o por cambios de ciclos hidrológicos, entre otros fenómenos (Introcaso, 2006).

La magnitud geométrica que define la altura del geoide con respecto a un elipsoide de referencia es la ondulación del geoide $\mathrm{N}$, que se mide a lo largo de la normal al elipsoide de referencia.

La ondulación del geoide $\mathrm{N}$ o altura geoidal, se puede calcular a partir de modelos geopotenciales globales de libre disponibilidad en centros de cálculos como el ICGEM. 
La fórmula de Bruns (Heiskanen y Moritz, 1967) relaciona la ondulación del geoide con el potencial perturbador T, mediante la expresión:

$$
N=\frac{T}{\gamma}
$$

Con las ecuaciones (7.1) y (2.27) la ondulación del geoide se expresa:

$$
N(r, \vartheta, \lambda)=\frac{G M}{\gamma \cdot r} \sum_{n=2}^{\infty}\left(\frac{a}{r}\right)^{n} \sum_{m=0}^{n}\left(\Delta \overline{C_{l m}} \cos \cdot m \lambda+\Delta \overline{S_{n m}} \cdot \operatorname{sen} \cdot m \lambda\right) \bar{P}_{n m}(\cos \vartheta)
$$

El análisis espectral de la descomposición en diferentes longitudes de onda permite, tener una idea sobre las distintas profundidades de los emplazamientos de las masas anómalas.

El modelo geopotencial EGM2008 contiene armónicos esféricos hasta el grado 2190 y orden 2159. Desde el calculador del ICGEM se han obtenido los valores de N para la zona de estudio, en una grilla regular con una resolución de un minuto de arco $(\sim 2 \mathrm{~km}$ de resolución espacial). El cálculo se realizó sin incluir el término cero.

A los fines de analizar el comportamiento de las ondulaciones del geoide se calcularon grillas con valores de $\mathrm{N}$ para diferentes grados: para el grado máximo del modelo 2190, y para grados menores: 360, 150, 120, 60 y 30. Los estadísticos de los valores de N para diferentes grados se resumen en la Tabla 7.1.

\begin{tabular}{|c|c|c|c|c|c|c|}
\hline & $\begin{array}{c}\text { Grado } \\
30 \\
\end{array}$ & $\begin{array}{c}\text { Grado } \\
60 \\
\end{array}$ & $\begin{array}{c}\text { Grado } \\
120 \\
\end{array}$ & $\begin{array}{c}\text { Grado } \\
150 \\
\end{array}$ & $\begin{array}{c}\text { Grado } \\
360 \\
\end{array}$ & $\begin{array}{c}\text { Grado } \\
2190 \\
\end{array}$ \\
\hline Num. Nodos Grilla & 141361 & 141361 & 141361 & 141361 & 141361 & 141361 \\
\hline Valor Máximo N [m] & 22.34 & 25.31 & 27.63 & 27.45 & 28.17 & 28.33 \\
\hline Valor Mínimo N [m] & 11.15 & 13.73 & 13.21 & 13.04 & 12.42 & 12.42 \\
\hline Rango N [m] & 11.19 & 11.58 & 14.42 & 14.41 & 15.75 & 15.91 \\
\hline Promedio N [m] & 18.39 & 18.61 & 18.50 & 18.50 & 17.60 & 18.52 \\
\hline Desviación estándar N [m] & \pm 2.74 & \pm 3.33 & \pm 3.35 & \pm 3.37 & \pm 3.45 & \pm 3.45 \\
\hline
\end{tabular}

Tabla 7.1.- Resumen estadístico de ondulación del geoide N del EGM2008.

Para visualizar el comportamiento espacial de los $\mathrm{N}$ para diferentes grados y su correlación con la topografía se han trazado isolíneas de N (para cada grado analizado) y se las superpuso sobre un mapa topográfico de la zona (Figura 7.1). Las isolíneas para cada grado se obtuvieron usando el método de interpolación Kriging lineal, en una grilla con resolución de un minuto de arco. 
En la Figura 7.1 se advierte que para el grado 30 (que corresponde a una longitud de onda de $1200 \mathrm{~km}$ ) las isolíneas de $\mathrm{N}$ muestran una tendencia de inclinación del geoide NorOeste a Sur-Este. Para el grado 60 (longitud de onda asociada de $600 \mathrm{~km}$ ) las isolíneas ya reflejan efectos de la topografía del sistema de los Andes al Oeste y de la cuenca oceánica hacia el Sur-Este. El grado 120 (longitud de onda asociada de $300 \mathrm{~km}$ ) y para los grados más altos (grado 150 hasta 2190) el trazado de isolíneas de la ondulación del geoide muestra los efectos de la topografía local. En particular, las isolíneas para el grado 2190 (longitud de onda asociada $18 \mathrm{~km}$ ) advierten un mayor grado de correlación con la topografía como se observa en las curvas cerradas en torno a los picos mas elevados del sistema de Los Andes.

De este análisis, se concluye, como ha de esperarse, que los grados más altos muestran los efectos de la corta longitud de onda topografía, mientras los grados más bajos reflejan efectos más profundos asociados con las largas longitudes de onda. Esto se analizará con más detalle en el punto siguiente. 


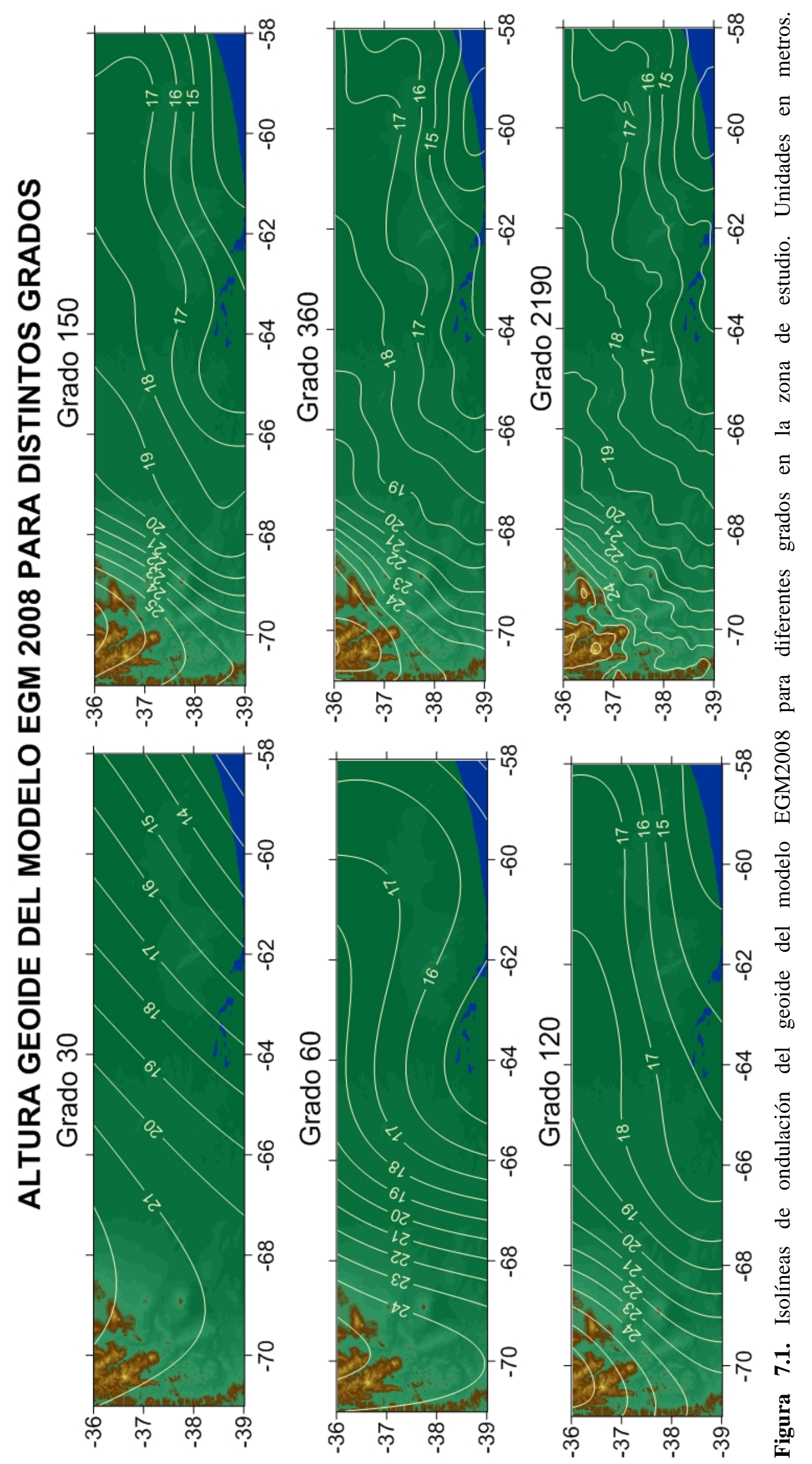




\subsection{Filtrado del Geoide}

Las ondulaciones del geoide $\mathrm{N}$, evidencian anomalías ocasionadas por anomalías de masa a diferentes profundidades.

El filtrado de $\mathrm{N}$ consiste en la separación de la señal del campo gravitacional en diferentes longitudes de onda, y con ello se logra operar sólo con las longitudes de onda que interesan. Con este propósito se considera la siguiente relación (Introcaso et al., 2005):

$$
N=N_{L L O}+N_{L O M}+N_{L O C}+N_{L O U C}
$$

donde:

$N$ : le corresponde la integración total de las diferentes longitudes de onda

$N_{L L O}$ : involucra anomalías de larga longitud de onda

$N_{L O M}$ : le corresponden las longitudes de onda intermedia

$N_{L O C}$ : le corresponden las longitudes de onda corta y

$N_{\text {LOUC: }}$ le corresponden las longitudes de onda ultra corta

En la ecuación (7.3) las ondulaciones de larga longitud de onda $\left(N_{L L O}\right)$ y media $\left(N_{L O M}\right)$ corresponden a los efectos profundos y muy profundos (por ejemplo manto inferior), a la ondulación corta $\left(N_{L O C}\right)$ se la vincula con efectos corticales y finalmente a la ultracorta $\left(N_{\text {LOUC }}\right)$ se la relaciona con efectos topográficos o bien con anomalías subsuperficiales menores (Torge, 1989; Introcaso, 2006). En la Tabla 7.2 se muestra la relación entre las diferentes longitudes de onda y las causas que las originan (Torge, 1989).

La descomposición espectral en diferentes longitudes de onda se relaciona con el grado del modelo geopotencial mediante la ecuación (2.25) y puede revelar las causas que la originan (Torge, 1989).

Tabla 7.2.-Relación entre las longitudes de onda del modelo geopotencial y las fuentes que la originan (Torge, 1989).

\begin{tabular}{l|c|c|l}
\hline & Grado $\mathrm{n}$ & Longitud de onda $\kappa$ & \multicolumn{1}{c}{ Fuentes que la causan } \\
\hline Onda larga & $2-36$ & $18000 \mathrm{~km}$ a $1000 \mathrm{~km}$ & \\
\hline Onda media & $37-180$ & $1000 \mathrm{~km}$ a $200 \mathrm{~km}$ & $\begin{array}{l}\text { Estructuras topográficas y geológicas } \\
\text { regionales (montañas, basamentos } \\
\text { Sedimentarios) }\end{array}$ \\
\hline Onda corta & $181-2000$ & $200 \mathrm{~km}$ a $18 \mathrm{~km}$ & $\begin{array}{l}\text { Topografía local y perturbaciones de } \\
\text { masa en subsuperficie. }\end{array}$ \\
\hline
\end{tabular}


El modelo geopotencial EGM2008 cuyo grado en términos de armónicos esféricos es 2190 contiene información hasta de la longitud de onda ultracorta.

Con la finalidad de filtrar la ondulación del geoide e identificar eventuales estructuras geológicas, se separan los efectos de corta longitud de onda truncando el desarrollo en serie de los primeros términos. En este caso se truncó el desarrollo para grado 120 (longitud de onda asociada $\approx 300 \mathrm{~km}$ ), obteniéndose una ondulación con el aporte $\mathrm{N}_{\text {LLO }}$ y $\mathrm{N}_{\mathrm{LOM}}$. Teniendo en cuenta la ecuación (7.3), se determinó una ondulación residual $\mathrm{N}_{\mathrm{R}}$ con la expresión (7.4)

$$
N_{R}=N-\left(N_{L L O}+N_{L O M}\right) .
$$

La ondulación residual $\mathrm{N}_{\mathrm{R}}$ contiene información sobre la corta y ultra corta longitud de onda, ya que se ha eliminado la información de la larga y media longitud de onda.

Para obtener la ondulación del geoide residual $\mathrm{N}_{\mathrm{R}}$ se utilizaron las grillas de $\mathrm{N}$ del modelo EGM2008 obtenidas en el punto 7.1, con grado máximo 2190 y grado 120. A partir de las dos grillas obtenidas del calculador del ICGEM, se aplicó la ecuación (7.4), resultando una nueva grilla con igual resolución a las anteriores y valores de ondulación residual. El resumen estadístico de esta grilla se muestra en la Tabla 7.3.

El rango de variación de las magnitudes del geoide residual es de aproximadamente 2.5m, con un máximo de $1.43 \mathrm{~m}$ y un mínimo de $-1 \mathrm{~m}$. La ondulación $\mathrm{N}_{\mathrm{R}}$ refleja los efectos de las masas más superficiales como la topografía, lo cual justifica la fuerte correlación de $\mathrm{N}_{\mathrm{R}}$ con la topografía, como se muestra en la Figura 7.2.

Tabla 7.3.- Resumen estadístico de ondulación del geoide residual del EGM2008.

\begin{tabular}{l|r}
\hline & $\mathrm{N}_{(\mathrm{Gr}: 2190)}-\mathrm{N}_{(\mathrm{Gr}: 120)}$ \\
\hline Num. Nodos Grilla & 141361 \\
\hline Valor Máximo [m] & 1.43 \\
\hline Valor Mínimo [m] & -1.04 \\
\hline Promedio [m] & 0.01 \\
\hline Desviación estándar [m] & \pm 0.43 \\
\hline
\end{tabular}

En la Figura 7.2 se advierten curvas cerradas con magnitudes altas y positivas de ondulación residual, en torno a los picos de mayor elevación de la topografía. Asimismo se advierten curvas cerradas y de valores negativos que corresponderían a sectores con defectos de masa. A partir de este filtrado se intenta identificar, a priori, la presencia de estructuras, las cuales se resumen en la Tabla 7.4 y se ubican espacialmente en la Figura 7.2. 


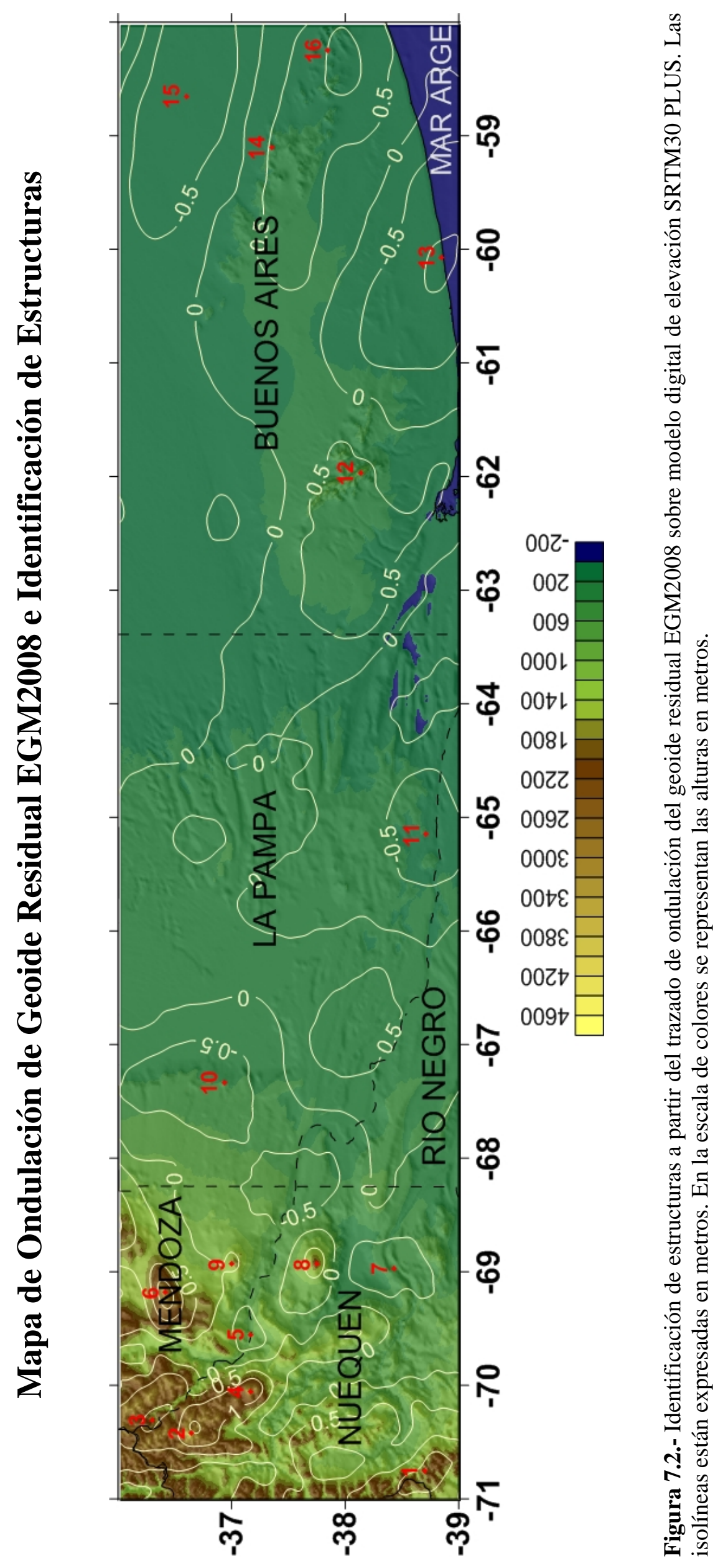


Tabla 7.4.- Estructuras identificadas a priori a partir de la ondulación residual $\mathrm{N}_{\mathrm{R}}$.

\begin{tabular}{c|c|c|c|c|c}
\hline Numero & $\begin{array}{c}\text { Longitud } \\
\mathbf{(})\end{array}$ & $\begin{array}{c}\text { Latitud } \\
(\mathbf{(})\end{array}$ & $\begin{array}{c}\text { N Residual } \\
(\mathbf{m})\end{array}$ & Nombre de la estructura & Provincia \\
\hline 1 & -70.7500 & -38.7000 & 1.68 & Cerro Las Lajas & Neuquén \\
\hline 2 & -70.4166 & -36.6500 & 1.47 & Volcán Domuyo & Neuquén \\
\hline 3 & -70.3000 & -36.3000 & 0.31 & Laguna Cari Lauquen -Río Barrancas & Mendoza \\
\hline 4 & -70.0500 & -37.1667 & 1.34 & Volcán Tromen & Neuquén \\
\hline 5 & -69.5500 & -37.1667 & -0.72 & Valle del Río Colorado & Mendoza \\
\hline 6 & -69.1833 & -36.4167 & 1.65 & Volcán Payún Matrú & Mendoza \\
\hline 7 & -68.9666 & -38.4333 & -0.64 & Emb. Cerros Colorados. R Neuquen & Neuquén \\
\hline 8 & -68.9333 & -37.7500 & 0.72 & Volcán Auca Mahuida & Neuquén \\
\hline 9 & -68.9333 & -37.0000 & 0.05 & Sierras de Chachahuen & Mendoza \\
\hline 10 & -67.3333 & -36.9333 & -0.8 & Bañados del Atuel y Salinas Grandes & La Pampa \\
\hline 11 & -65.1500 & -38.7166 & -0.73 & Sierra de Pichi Mahuida & La Pampa \\
\hline 12 & -61.9666 & -38.1333 & 0.69 & Sierras de la Ventana & Buenos Aires \\
\hline 13 & -60.0666 & -38.8500 & -1.06 & Cuenca de Claromecó & Buenos Aires \\
\hline 14 & -59.1000 & -37.3500 & 0.54 & Sierras de Tandil & Buenos Aires \\
\hline 15 & -58.6500 & -36.6000 & -0.95 & Cuenca del Salado & Buenos Aires \\
\hline 16 & -58.2500 & -37.8500 & 1.01 & Sierras de Balcarce & Buenos Aires \\
\hline
\end{tabular}

\subsection{Correlación de la ondulación del geoide residual con anomalías de aire libre y}

\section{Bouguer}

Se efectuó la correlación espacial entre las anomalías de aire libre y Bouguer obtenidas en el Capítulo IV con la ondulación residual $\mathrm{N}_{\mathrm{R}}$ del modelo EGM2008, con el fin de analizar a priori el estado isostático de las estructuras identificadas previamente.

Se tomaron valores de AAL y ABPC de las estructuras identificadas, teniendo en cuenta que las magnitudes de las anomalías se tratan como valores medios para el sector de emplazamiento de la misma. Los resultados se detallan en la Tabla 7.5.

Tabla 7.5.- Anomalías de aire libre y Bouguer de las estructuras identificadas.

\begin{tabular}{c|c|c|c|c|c|c|c}
\hline $\mathbf{N}^{\mathbf{0}}$ & Nombre & $\begin{array}{c}\text { Longitud } \\
(\mathbf{(})\end{array}$ & $\begin{array}{c}\text { Latitud } \\
(\mathbf{o})\end{array}$ & $\begin{array}{c}\mathbf{N}_{\mathbf{R}} \\
(\mathbf{m})\end{array}$ & $\begin{array}{c}\text { AAL } \\
(\mathbf{m G a l})\end{array}$ & $\begin{array}{c}\text { AB } \\
(\mathbf{m G a l})\end{array}$ & $\begin{array}{c}\text { Altura } \\
(\mathbf{m})\end{array}$ \\
\hline 1 & Cerro Las Lajas & -70.7500 & -38.7000 & 1.68 & 19 & -85 & 2463 \\
\hline 2 & Volcán Domuyo & -70.4166 & -36.6500 & 1.47 & 5 & -130 & 3825 \\
\hline 3 & Laguna Cari Lauquen & -70.3000 & -36.3000 & 0.31 & 0 & -147 & 1833 \\
\hline 4 & Volcán Tromen & -70.0500 & -37.1667 & 1.34 & 30 & -115 & 3980 \\
\hline 5 & Valle del Río Colorado & -69.5500 & -37.1667 & -0.72 & -14 & -106 & 771 \\
\hline 6 & Volcán Payún Matrú & -69.1833 & -36.4167 & 1.65 & 32 & -132 & 3680 \\
\hline 7 & Emb. Cerros Colorados. & -68.9666 & -38.4333 & -0.64 & -11 & -57 & 447 \\
\hline 8 & Volcán Auca Mahuida & -68.9333 & -37.7500 & 0.72 & -11 & -56 & 2253 \\
\hline 9 & Sierras de Chachahuen & -68.9333 & -37.0000 & 0.05 & 10 & -84 & 2050 \\
\hline 10 & Bañados del Atuel & -67.3333 & -36.9333 & -0.8 & -22 & -68 & 300 \\
\hline 11 & Sierras Mahuidas & -65.1500 & -38.7166 & -0.73 & -18 & -39 & 200 \\
\hline 12 & Sierras de la Ventana & -61.9666 & -38.1333 & 0.69 & 9 & -19 & 1239 \\
\hline 13 & Cuenca de Claromecó & -60.0666 & -38.8500 & -1.06 & -35 & -25 & 14 \\
\hline 14 & Sierras de Tandil & -59.1000 & -37.3500 & 0.54 & 35 & 102 & 524 \\
\hline 15 & Cuenca del Salado & -58.6500 & -36.6000 & -0.95 & 17 & 11 & 48 \\
\hline 16 & Sierras de Balcarce & -58.2500 & -37.8500 & 1.01 & 37 & 24 & 300 \\
\hline
\end{tabular}


Para el análisis de la correlación espacial entre las AAL y las ABPC con las ondulaciones del geoide residual, se trazaron las isolíneas de $\mathrm{N}_{\mathrm{R}}$ sobre los mapas de AAL y ABPC obtenidas en el Capítulo IV (Figura 7.3).
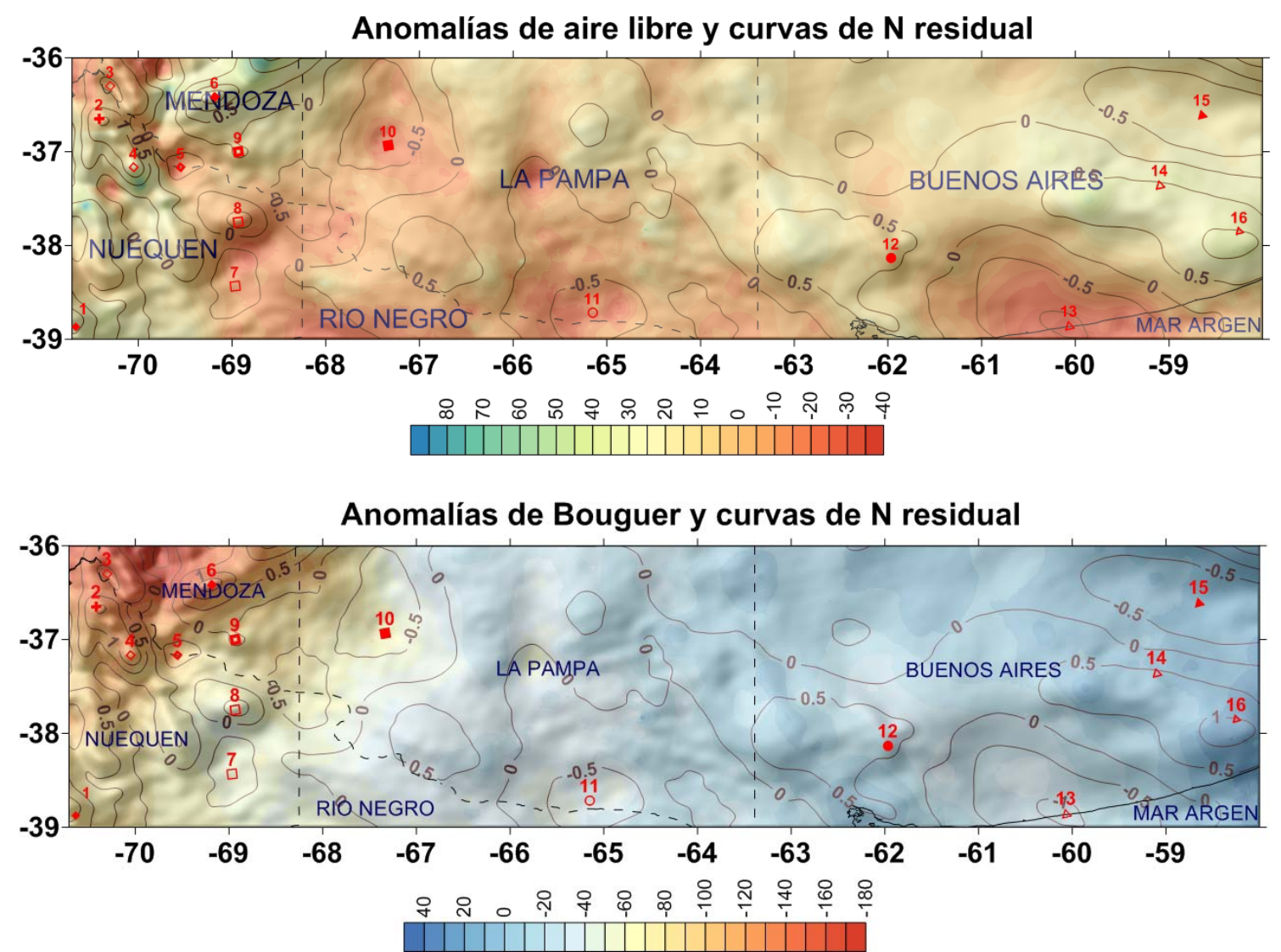

Figura 7.3.- Isolíneas de ondulación del geoide residual EGM2008 sobre mapa de anomalías de aire libre (arriba) y anomalías de Bouguer (abajo). La escala de color representa unidades de mGal. La ondulación residual $\mathrm{N}$ está expresada en metros.

De acuerdo a lo expresado anteriormente para una topografía compensada, los valores de AAL son cercanos a cero y las AB correlacionan negativamente con la altura. A continuación se analiza cada estructura identificada en relación con las anomalías gravimétricas.

\section{Estructura 1.- Cerro Las Lajas (Provincia de Neuquén)}

Corresponde al sistema de Los Andes Meridionales, en el extremo Sur-Oeste de la zona de trabajo. La altura de esta estructura alcanza los $2463 \mathrm{~m}$. El $\mathrm{N}_{\mathrm{R}}$ es de $+1.68 \mathrm{~m}$ que se corresponde claramente con la masa topográfica del sistema. Los valores de AAL $+19 \mathrm{mGal}$ y AB de -85 mGal, muestran una tendencia al equilibrio isostático. 


\section{Estructura 2.-Volcán Domuyo (Provincia de Neuquén)}

Corresponde al sistema de Los Andes Meridionales al Oeste de la zona de trabajo. La altura de esta estructura alcanza los $3825 \mathrm{~m}$. El $\mathrm{N}_{\mathrm{R}}$ es de $+1.47 \mathrm{~m}$ que se corresponde con la masa topográfica del sistema. Los valores de AAL +5 mGal y AB de -130 mGal, reflejan a priori que la estructura estaría en equilibrio isostático.

Estructura 3.-Laguna Cari Lauquen, Valle del Río Barrancas, afluente del Río Colorado (Provincia de Mendoza)

Corresponde al sistema de drenaje de Los Andes al Oeste. La altura del valle es de $1800 \mathrm{~m}$ aproximadamente. El $\mathrm{N}_{\mathrm{R}}$ es de $+0.31 \mathrm{~m}$ que se corresponde con la masa topográfica del sistema. Los valores de AAL $0 \mathrm{mGal}$ y AB de $-147 \mathrm{mGal}$, reflejan a priori que la estructura estaría en equilibrio isostático.

\section{Estructura 4.- Volcán Tromen (Provincia de Neuquén)}

Corresponde al sistema de Los Andes Meridionales al Oeste. La altura de esta estructura alcanza los $3980 \mathrm{~m}$. El $\mathrm{N}_{\mathrm{R}}$ es de $+1.34 \mathrm{~m}$ que se corresponde con la masa topográfica del sistema. Los valores de AAL $+30 \mathrm{mGal}$ y $\mathrm{AB}$ de $-115 \mathrm{mGal}$, reflejan a priori una tendencia al equilibrio isostático.

\section{Estructura 5.- Valle del Río Colorado (Provincia de Mendoza)}

Corresponde al sistema de drenaje de Los Andes Meridionales al Oeste. La altura del valle es de $770 \mathrm{~m}$ aproximadamente. El $\mathrm{N}_{\mathrm{R}}$ es de $-0.72 \mathrm{~m}$ que se correspondería con un defecto de masa. Los valores de AAL -14 mGal y AB de -106 mGal, reflejan a priori una tendencia al equilibrio isostático.

\section{Estructura 6.- Volcán Payún Matrú (Provincia de Mendoza)}

Corresponde al sistema de Los Andes Meridionales al Oeste. La altura de esta estructura alcanza los $3680 \mathrm{~m}$. El $\mathrm{N}_{\mathrm{R}}$ es de $+1.65 \mathrm{~m}$ que se corresponde con la masa topográfica del sistema. Los valores de AAL $+32 \mathrm{mGal}$ y $\mathrm{AB}$ de $-132 \mathrm{mGal}$, reflejan a priori una tendencia al equilibrio isostático.

\section{Estructura 7.- Embalse Cerros Colorados, Río Neuquén (Provincia de Neuquén)}

Corresponde al sistema de drenaje de Los Andes Meridionales al Oeste. La altura de esta estructura alcanza los $447 \mathrm{~m}$. El $\mathrm{N}_{\mathrm{R}}$ es de $-0.64 \mathrm{~m}$ que corresponde un defecto de masa. Los valores de AAL $-11 \mathrm{mGal}$ y $\mathrm{AB}$ de $-57 \mathrm{mGal}$, reflejan a priori una tendencia al equilibrio isostático. 


\section{Estructura 8.- Volcán Auca Mahuida (Provincia de Mendoza)}

Corresponde al sistema de Los Andes Meridionales al Oeste. La altura de esta estructura alcanza los $2253 \mathrm{~m}$. El $\mathrm{N}_{\mathrm{R}}$ es de $+0.72 \mathrm{~m}$ que se corresponde con la masa topográfica del sistema. Los valores de AAL - $11 \mathrm{mGal}$ y AB de $-56 \mathrm{mGal}$, reflejan a priori una tendencia al equilibrio isostático.

\section{Estructura 9.- Sierras de Chachahuén (Provincia de Mendoza)}

Corresponde al sistema de Los Andes Meridionales al Oeste. La altura de esta estructura alcanza los $2050 \mathrm{~m}$. El $\mathrm{N}_{\mathrm{R}}$ es de $+0.05 \mathrm{~m}$ indica que las masas topográficas están compensadas con las raíces corticales. Los valores de AAL $+10 \mathrm{mGal}$ y AB de $-84 \mathrm{mGal}$, reflejan que la estructura estaría en equilibrio isostático.

\section{Estructura 10.- Bañados del Atuelo Salinas Grandes (Provincia de La Pampa)}

Cuenca sedimentaria que se orienta según una falla tectónica en el sector Oeste de la provincia de La Pampa, formando una serie de humedales llamados Bañados del Atuel. La cuenca continua hacia el Sur con Las Salinas Grandes o Gran Salitral La altura de esta estructura alcanza los $300 \mathrm{~m}$. El $\mathrm{N}_{\mathrm{R}}$ es de $-0.80 \mathrm{~m}$ que se corresponde con un defecto de masa. Los valores de AAL -22 mGal y AB de -68 mGal, reflejan a priori una tendencia al equilibrio isostático.

\section{Estructura 11.- Sierras Mahuidas (Provincia de La Pampa)}

Las Sierras Mahuidas son un sistema serrano antiguo muy erosionado ubicado en la provincia de La Pampa. Comprende las sierras Choique Mahuida y Pichi Mahuida. La altura de esta estructura no supera los $200 \mathrm{~m}$. El $\mathrm{N}_{\mathrm{R}}$ es de - $0.73 \mathrm{~m}$ que se corresponde con un defecto de masa. Los valores de AAL -18 mGal y AB de -39 mGal, reflejan a priori una tendencia al equilibrio isostático.

\section{Estructura 12.- Sierras de la Ventana (Provincia de Buenos Aires)}

Corresponde al sistema de la Ventana al Sur-Oste de la provincia de Buenos Aires. La altura de esta estructura alcanza los $1239 \mathrm{~m}$. El $\mathrm{N}_{\mathrm{R}}$ es de $+0.69 \mathrm{~m}$ que se corresponde con la masa topográfica del sistema. Los valores de AAL +9 mGal y AB de -19 mGal, reflejan a priori que la estructura estaría en equilibrio isostático.

\section{Estructura 13.-Cuenca de Claromecó (Provincia de Buenos Aires)}

La Cuenca de Claromecó es una cuenca sedimentaria y se ubica en el extremo Sur de la provincia de Buenos Aires. La altura de esta estructura alcanza los $14 \mathrm{~m}$. El $\mathrm{N}_{\mathrm{R}}$ es de $-1.06 \mathrm{~m}$ que se corresponde con un defecto de masa de la cuenca. Los valores de AAL -35 mGal y AB de $-25 \mathrm{mGal}$, no reflejan a priori que la estructura esté en equilibrio, sin embargo estudios de la 
zona indican que la estructura se corresponde con un modelo de corteza distensiva compensada isostáticamente en el sistema de Airy (Introcaso y Ruiz, 2005).

\section{Estructura 14.- Sierras de Tandil (Provincia de Buenos Aires)}

Corresponde al sistema de Tandilia al Sur-Este de la provincia de Buenos Aires. La altura de esta estructura alcanza los $524 \mathrm{~m}$. El $\mathrm{N}_{\mathrm{R}}$ es de $+0.54 \mathrm{~m}$ que se corresponde con la masa topográfica del sistema. Sobre las Sierras de Tandil las anomalías de Bouguer son predominantemente positivas (Fig. 4.2) variando entre -15 y +35 mGal (Kostadinoff, 1995 y Del Cogliano, 2006). Consideraciones geológicas explican las anomalías AB positivas a partir de la presencia de -masas anómalas en la corteza (Del Cogliano, 2006) y demuestran el estado de equilibrio de la estructura.

\section{Estructura 15.- Cuenca del Salado (Provincia de Buenos Aires)}

Corresponde a la Cuenca sedimentaria del Salado ubicada en el extremo Nor-Este de la provincia de Buenos Aires. La altura de esta estructura alcanza los $48 \mathrm{~m}$. El $\mathrm{N}_{\mathrm{R}}$ es de $-0.95 \mathrm{~m}$ que se corresponde con un defecto de masa. Los valores de AAL $+17 \mathrm{mGal}$ y AB de $+11 \mathrm{mGal}$, reflejan a priori una tendencia que se correspondería con una sobrecompensación. (Introcaso y Ramos, 1984; Introcaso, 1993; Introcaso 1997; Introcaso y Ruiz, 2005).

\section{Estructura 16.- Sierras de Balcarce (Provincia de Buenos Aires)}

Corresponde al sistema de Tandilia al Este de la provincia de Buenos Aires. La altura de esta estructura alcanza los $300 \mathrm{~m}$. El $\mathrm{N}_{\mathrm{R}}$ es de $+1.01 \mathrm{~m}$ que se corresponde con la masa topográfica del sistema. Los valores de AAL +37 mGal y AB de -24 mGal, muestran un comportamiento similar a las Sierras de Tandil, ambas sierras pertenecen al sistema de Tandilla. En consecuencia la estructura no estaría compensada según un modelo isostático (Del Cogliano, 2006).

\subsection{Conclusiones del capítulo}

El análisis espectral de la ondulación del geoide $\mathrm{N}$ permite conocer las grandes contribuciones de masa y detectar anomalías a diferentes profundidades.

Con el filtrado del geoide se logró eliminar las masas profundas e identificar estructuras geológicas. A partir de las estructuras identificadas y correlacionadas con mapas de anomalías de aire libre y Bouguer se infiere a priori el estado isostático de las mismas. 
Capítulo VIII

\section{Análisis de las características corticales de estructuras geológicas a partir de ondulaciones del geoide isostático}

En este capítulo se especula sobre las características corticales de estructuras geológicas en equilibrio isostático a partir de la interpretación de desviaciones del geoide respecto del elipsoide de referencia. Los resultados obtenidos con este nuevo enfoque complementan y/o validan aquellos obtenidos a partir de análisis isostático tradicionales.

\subsection{Introducción}

Tradicionalmente los análisis isostáticos de estructuras geológicas se realizaron con anomalías de gravedad isostáticas. Dichas anomalías se calculan a partir de las anomalías de aire libre, aplicando dos correcciones, una por el efecto de la masa topográfica y la otra por efecto de la masa compensada teórica de acuerdo a un modelo isostático. Actualmente empezaron a tenerse en cuenta las ondulaciones del geoide para este tipo de estudios (Del Cogliano, 2006; Crovetto et al., 2006; Cornaglia, 2007).

Las ondulaciones del geoide generadas por una estructura teórica isostáticamente compensada pueden compararse con las ondulaciones del geoide observadas sobre la estructura real (en longitudes de onda compatibles con su ancho) y de esta forma disponer de otra vía de análisis isostático (Crovetto et al., 2006).

La información referida a la isostasia se corresponderá con longitudes de onda no mayores a unos pocos cientos de kilómetros, como consecuencia las anomalías de densidad en las profundidades de la Tierra deben ser previamente eliminadas (longitudes de onda medias y largas). Comparando el geoide observado y debidamente filtrado con el correspondiente a un modelo cortical perfectamente compensado será posible inferir sobre las características de la corteza (Del Cogliano, 2006). 


\subsection{Ondulación del Geoide e Isostasia}

Considerando que el potencial gravitatorio de la Tierra real $V_{\text {real }}$ puede expresarse como la suma del potencial producido por la Tierra regularizada $V_{\text {reg }}$ en el sentido isostático y un potencial perturbador isostático $T_{\text {isost }}$ producto de la topografía y el correspondiente modelo de compensación, es posible expresar (Del Cogliano, 2006):

$$
V_{\text {real }}=V_{\text {reg }}+T_{\text {isost }}+v_{I}
$$

De donde:

$$
T_{\text {isost }}=V_{\text {real }}-V_{\text {reg }}-V_{I}
$$

donde $v_{I}$ representa los desvíos del modelo isostático y los errores en la evaluación del mismo.

Si consideramos en el modelo de compensación de Airy, una estructura de referencia, con una corteza normal de espesor t, densidad de la corteza $\rho_{\mathrm{c}}$, y la densidad del manto $\rho_{\mathrm{m}}$; una estructura de altura H será compensada por la presencia de raíces de espesor r (ecuación 6.3), tal que:

$$
r=\frac{\rho_{c} H}{\rho_{m}-\rho_{c}}
$$

Se puede demostrar que el potencial perturbador isostático $T_{\text {isost }}$ producido por la masa topográfica de una estructura de altura H y raíz r será (Del Cogliano, 2006):

$$
T_{\text {isost }}=-2 \pi G \rho_{c} H\left[\frac{H}{2}+t+\frac{r}{2}\right]
$$

Aplicando la fórmula de Bruns (ecuación 7.1) en la ecuación (8.4):

$$
N_{\text {isost }}=\frac{2 \pi G \rho_{c} H}{\gamma}\left[\frac{H}{2}+t+\frac{r}{2}\right]
$$

La ecuación (8.5) resuelve la ondulación isostática $N_{\text {isost }}$ para una estructura compensada (Haxby y Turcotte, 1978) en términos de una función que depende únicamente de la altura de la estructura en estudio, establecidas las densidades y espesor cortical normal que serán asumidos como valores fijos 


\subsection{Análisis isostático de estructuras}

Es posible especular sobre las características de la corteza comparando el geoide observado y debidamente filtrado con el correspondiente a un modelo cortical perfectamente compensado (Del Cogliano, 2006).

Para la comparación tomaremos como geoide observado el obtenido del modelo geopotencial global EGM2008 y como geoide isostático el calculado con la ecuación (8.5), usando como parámetros de referencia (densidades y espesor de corteza) los correspondientes a la estructura.

La ondulación del geoide observada $N_{\text {obs }}$, puede escribirse como la suma de una componente regional $N_{\text {reg }}$ que refleja el comportamiento predominante de la zona y otra residual $N_{\text {res }}$ ligada a las particularidades de la estructura analizada. $N_{\text {obs }}$ se estima a través del modelo geopotencial al que designamos $N_{\text {mod }}$ y puede expresarse como:

$$
N_{\text {mod }}=N_{\text {reg }}+N_{\text {res }}+v_{n}
$$

donde $v_{\mathrm{n}}$ representa los errores del modelo geopotencial.

$$
N_{\text {res }}=N_{\text {mod }}-N_{\text {reg }}-v_{n}
$$

Si estas particularidades son atribuidas a las discrepancias entre la corteza real y la corteza regularizada, es posible asimilar la ondulación residual a la isostática (Del Cogliano, 2006).

Luego, si existe equilibrio isostático:

$$
N_{\text {res }}+v_{n} \cong N_{\text {isost }}+v_{I}
$$

donde $v_{\mathrm{I}}$ representa los desvíos del modelo isostático y los errores en la evaluación del mismo.

$$
v_{I}=N_{r e s}+v_{N}-N_{\text {isost }}
$$

Una vez eliminada la componente isostática de la ondulación residual, de acuerdo a (8.9), los valores $v_{I}$ muestran el eventual apartamiento de la estructura analizada del modelo isostático adoptado (Del Cogliano, 2006). 


\subsection{Estructura de Tandil}

Se escogió para este análisis la estructura de Tandil identificada en el Capítulo VII. El análisis de realizó sobre un perfil que secciona la estructura con una longitud de 220 km y un acimut de $129^{\circ}$ (Figura 8.1).

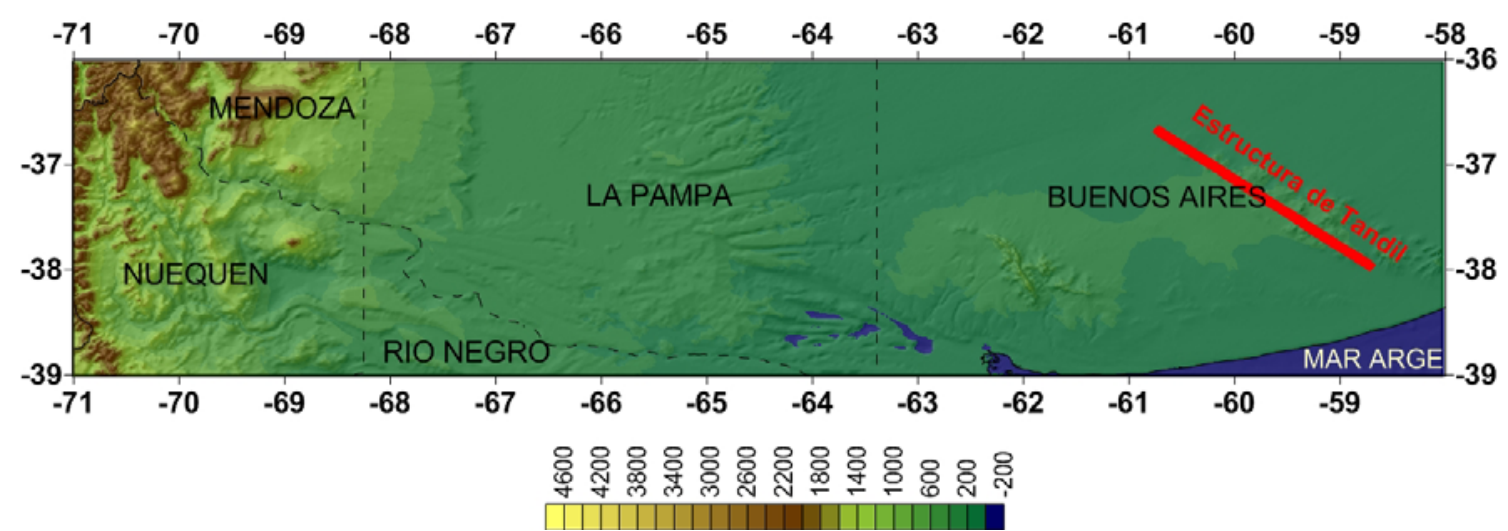

Figura 8.1.- Ubicación del perfil de la estructura de Tandil sobre mapa topográfico. Las unidades de la escala de color están en metros.

\section{Ondulación geoide isostático}

La estructura de Tandil está ubicada sobre el cratón del Río de La Plata, que se extiende desde Uruguay al Norte hasta la región de Tandilia al Sur. Los espesores de los cratones típicamente varían entre 40 y $45 \mathrm{~km}$. Una densidad de $2.84 \mathrm{gr} / \mathrm{cm} 3$ es considerada un valor medio para cortezas de estructuras cratónicas como Tandilla y es coherente con los resultados aportados por el modelo de corteza CRUST 5.1 (Mooney et al., 1998). En consecuencia, se asume tentativamente una corteza de espesor próximo a $42 \mathrm{~km}$ con una densidad promedio de $2.84 \mathrm{gr} / \mathrm{cm}^{3}$ sobre un manto de densidad $3.24 \mathrm{gr} / \mathrm{cm}^{3}$ (Del Cogliano, 2006).

Usando la ecuación (8.5) se obtuvieron las ondulaciones isostáticas $N_{\text {isost }}$ en el modelo isostático de Airy (Fowler, 2005). Las alturas fueron tomadas del modelo digital de elevaciones DTM2006. Como parámetros de referencia para la estructura de Tandil se tomaron los siguientes valores (Del Cogliano, 2006):

$\rho_{\mathrm{c}}$ densidad de la corteza: $2.84 \mathrm{~g} / \mathrm{cm}^{3}$,

$\rho_{\mathrm{m}}$ densidad del manto de $3.24 \mathrm{~g} / \mathrm{cm}^{3}$,

t espesor de corteza de referencia: $42 \mathrm{~km}$.

En la Figura 8.2 y Figura 8.3 se muestra el perfil topográfico y las ondulaciones del geoide isostático, respectivamente. Las ondulaciones del geoide isostático, siguen la forma de la 
topografía del perfil analizado, lo cual muestra que la ondulación isostática muestra los efectos de las masas topográficas.

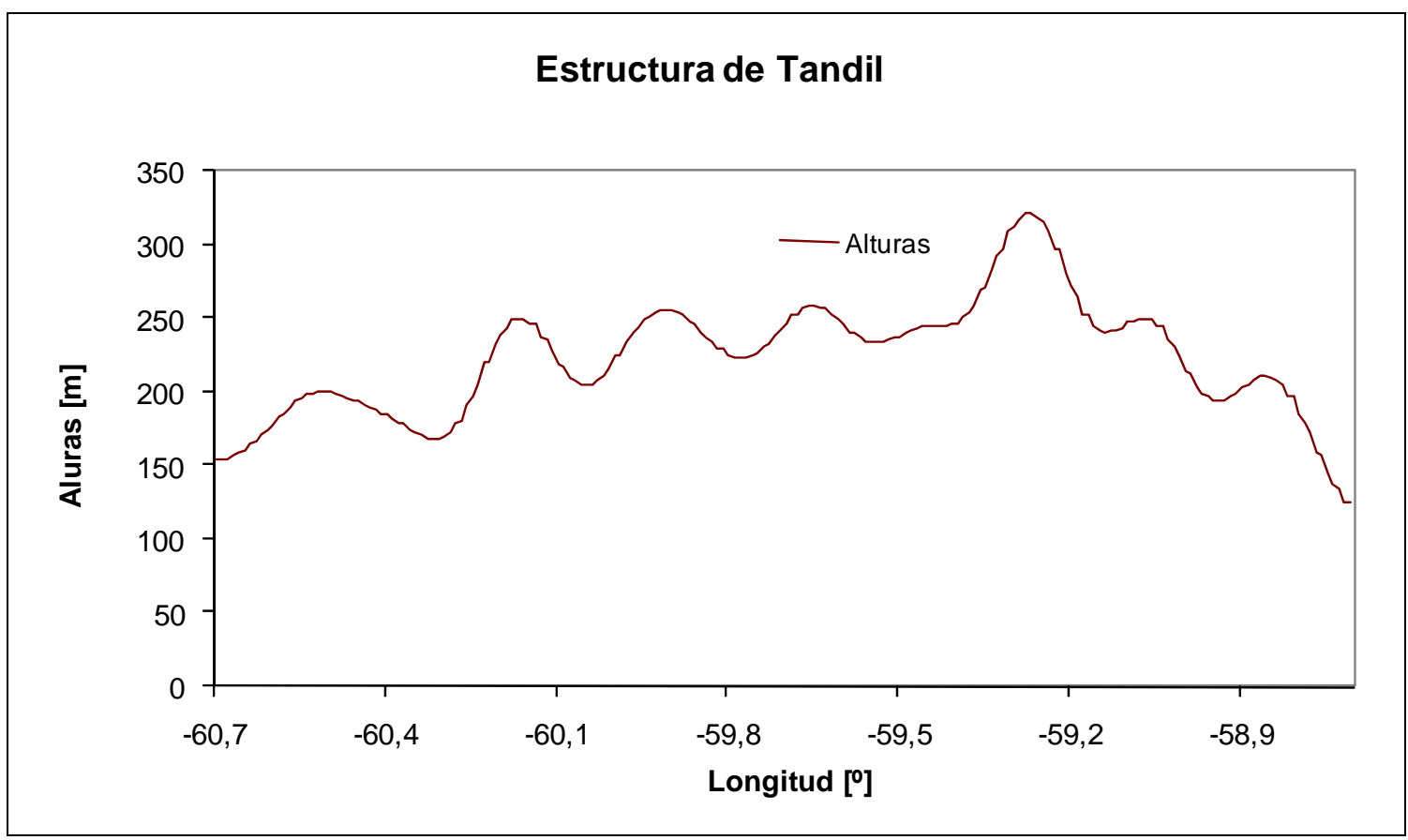

Figura 8.2.- Perfil topográfico del modelo DTM2006 sobre sección de la estructura de Tandil.

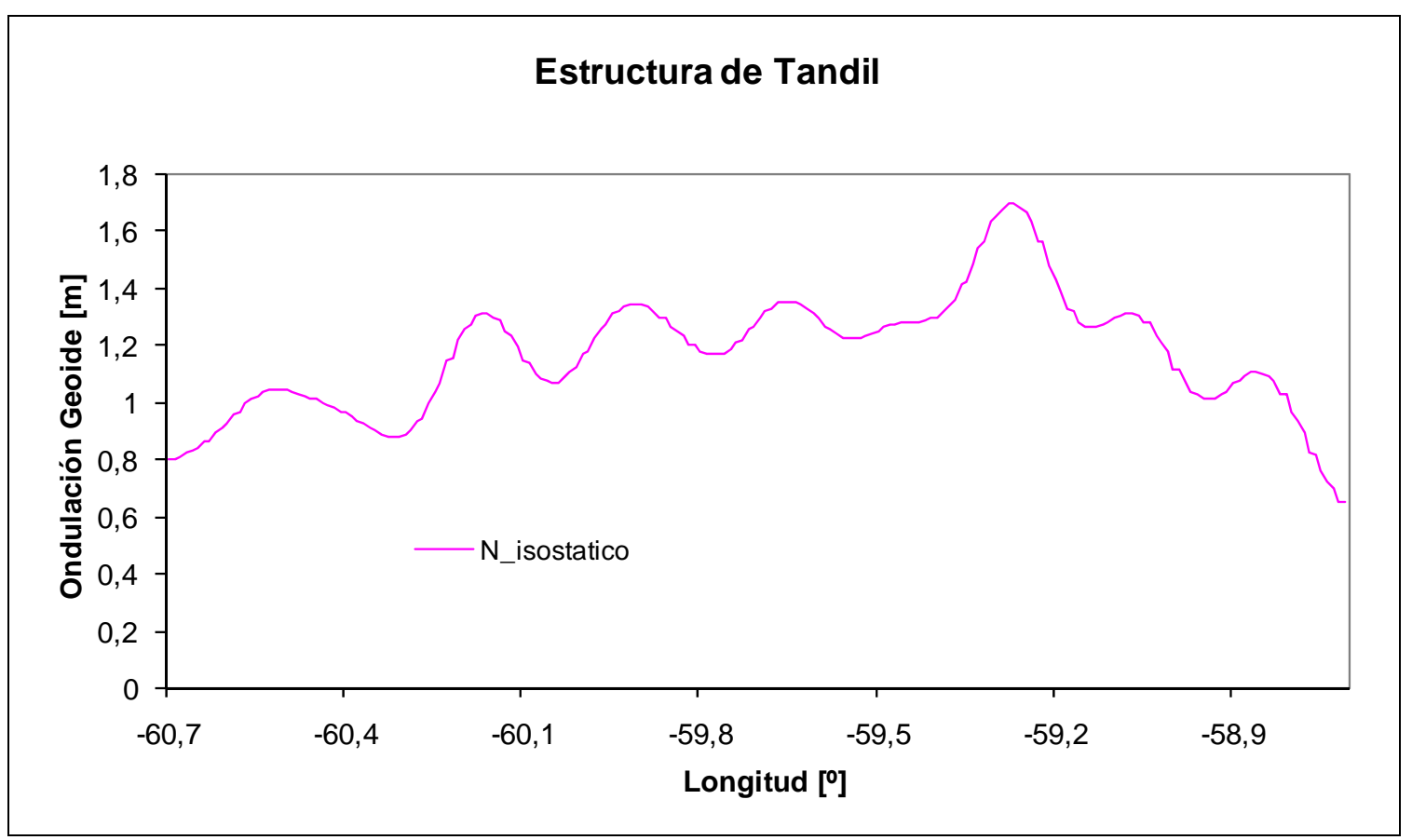

Figura 8.3.- Ondulación del geoide isostatico sobre sección de la estructura de Tandil. 
Para obtener el $N_{\text {res }}$ se utilizó la ecuación (8.7). El valor de ondulación del geoide $N_{\text {mod }}$ se obtuvo del modelo geopotencial EGM2008 completo, es decir hasta el grado 2190. Como ondulación del geoide regional $N_{\text {reg }}$ se tomó la ondulación resultante del modelo geopotencial truncado el desarrollo en grado 36 (longitud de onda asociada $\approx 1110 \mathrm{~km}$ y resolución $550 \mathrm{~km}$ ) con lo cual se filtran las estructuras mayores que 500 km. La Figura 8.4 muestra la ondulación del geoide residual obtenido con la ecuación (8.7).

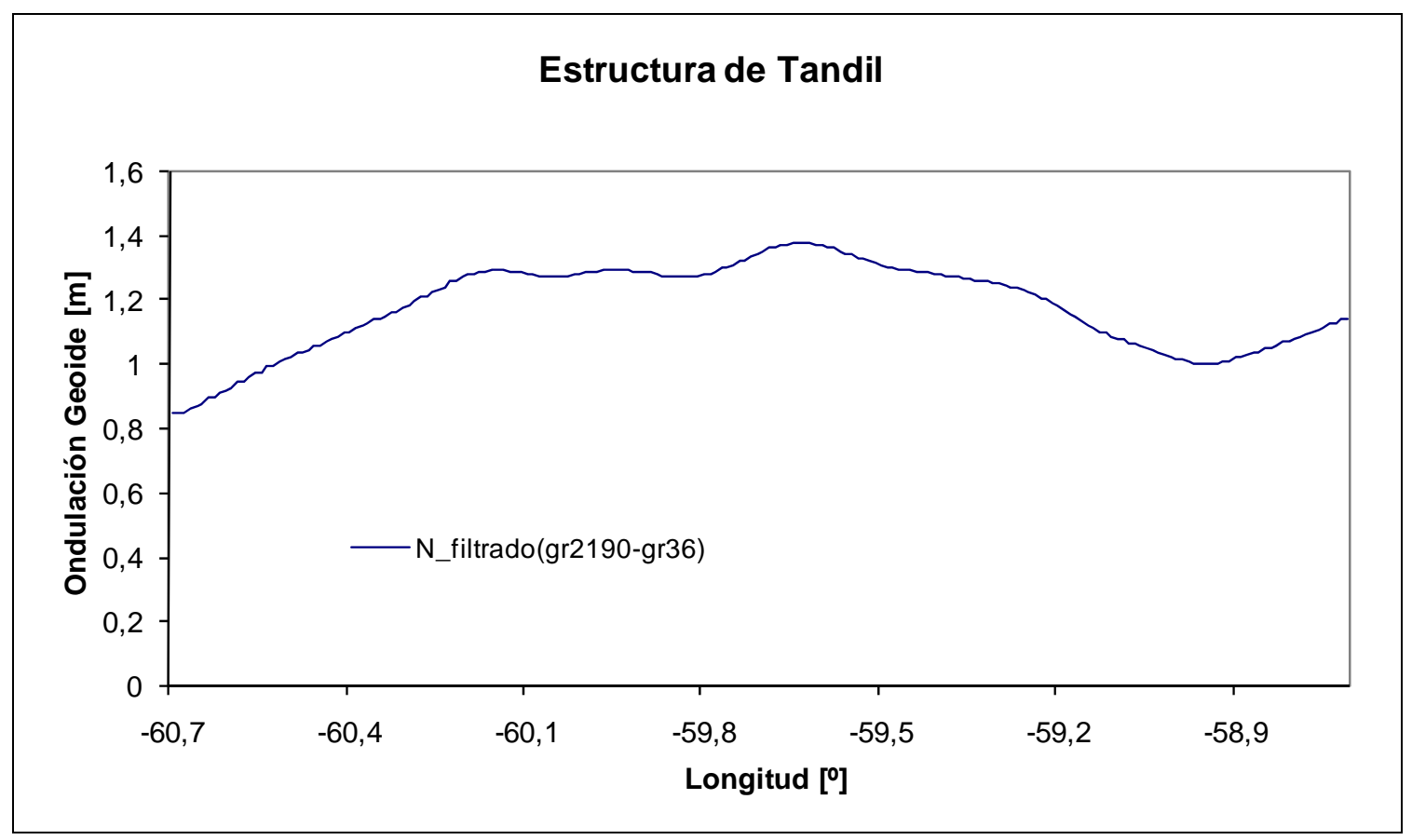

Figura 8.4.- Ondulación del geoide residual sobre la sección de Estructura de Tandil.

\subsection{Análisis de resultados}

A partir de la ecuación (8.9) se obtuvieron los desvíos que muestran el apartamiento de la estructura analizada del modelo isostatico adoptado. En la Tabla 8.1 se muestra el resumen estadístico de los desvíos. En la misma se advierte que la ondulación del geoide isostático tiene un buen ajuste respecto a la ondulación del geoide residual (filtrado) del modelo EGM2008, como lo muestra el promedio de los desvíos es de $0.01 \mathrm{~m}$ y la desviación estándar de $0.17 \mathrm{~m}$. El rango de los desvíos es 0.94 m, variando entre 0.49 m y -0.46 m (Figura 8.5 y 8.6). 


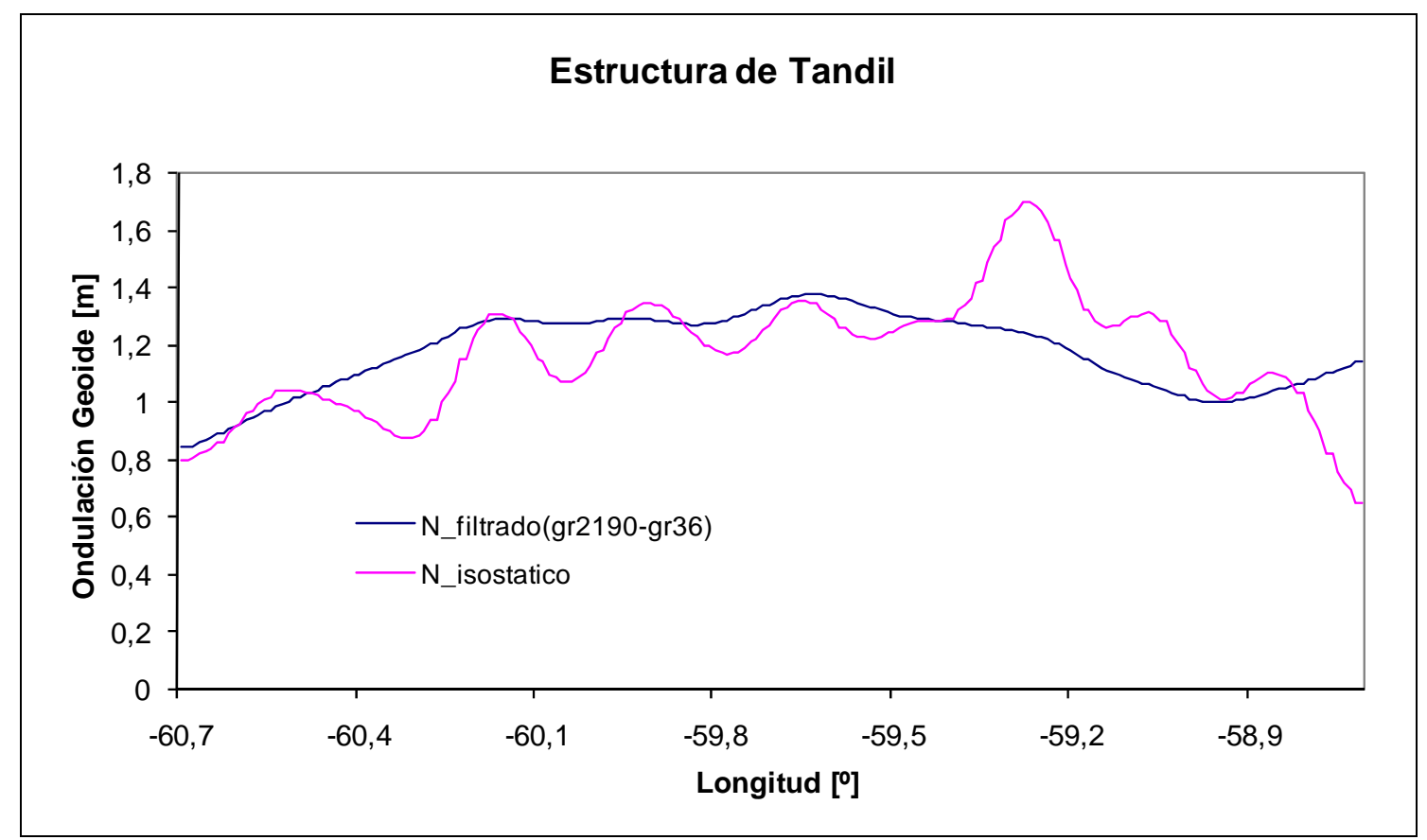

Figura 8.5.- Ondulación geoide residual e isostático sobre sección Tandil.

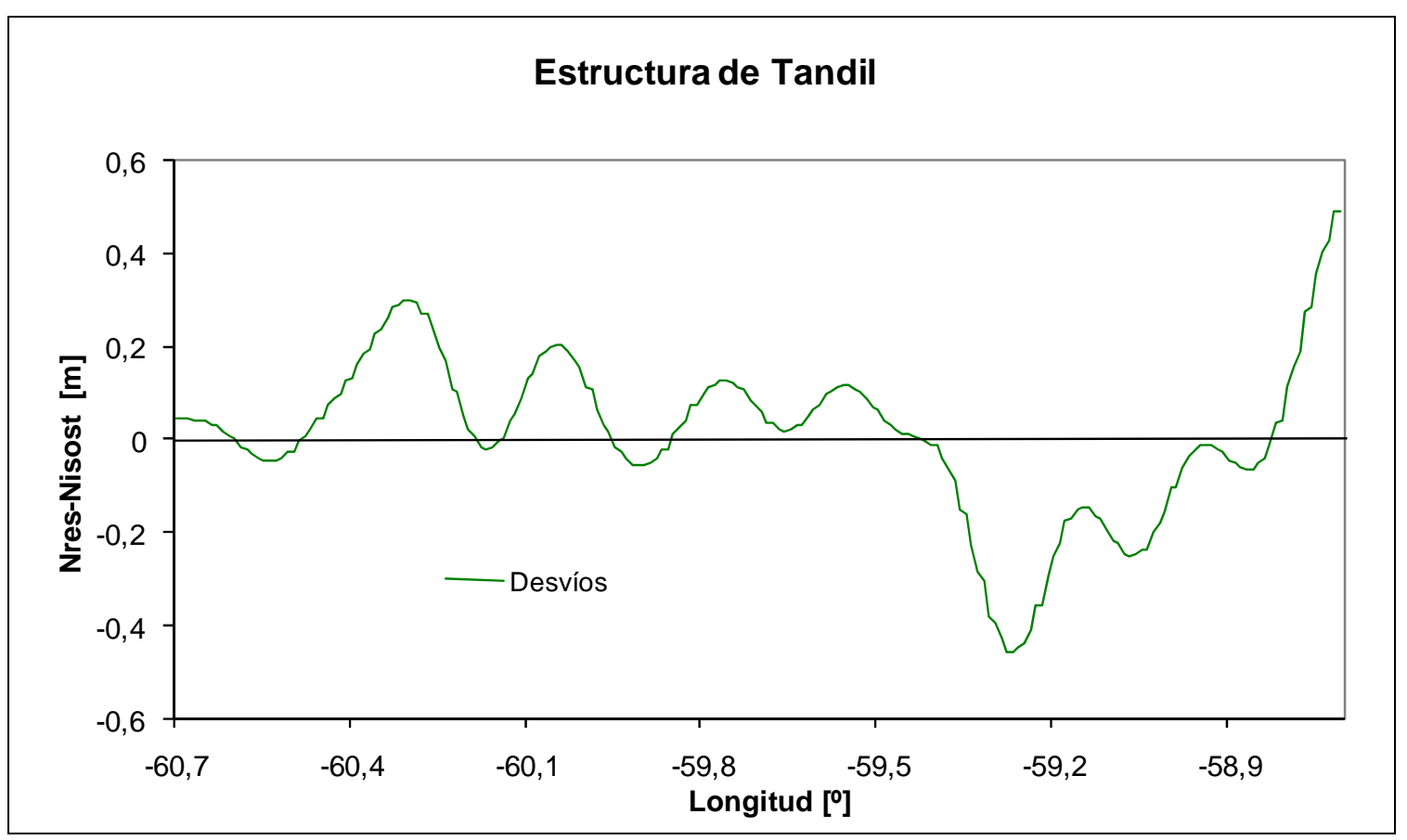

Figura 8.6.- Desvíos (ondulación geoide residual menos geoide isostatico) sobre sección Tandil.

Los resultados obtenidos muestran que los parámetros usados para el modelo teórico de corteza en la estructura de Tandil son los apropiados, corroborando además que la estructura estaría en equilibrio isostático.

Tabla 8.1.- Resumen estadístico de los desvíos entre geoide isostático y el geoide residual. 


\begin{tabular}{l|c|c|c}
\hline & $\begin{array}{c}\text { Ondulación } \\
\text { Isostática }\end{array}$ & $\begin{array}{c}\text { Ondulacion geoide } \\
\text { residual } \\
(\mathrm{n}=2190-\mathrm{n}=36)\end{array}$ & $\begin{array}{c}\text { Desvíos } \\
v_{I}=N_{\text {res }}-N_{\text {isost }}\end{array}$ \\
\hline Máximo [m] & 1.70 & 1.37 & 0.49 \\
\hline Mínimo [m] & 0.65 & 0.84 & -0.46 \\
\hline Rango [m] & 1.04 & 0.53 & 0.94 \\
\hline Promedio [m] & 1.16 & 1.17 & 0.01 \\
\hline Desv. estándar [m] & \pm 0.21 & \pm 0.14 & \pm 0.17 \\
\hline
\end{tabular}

\subsection{Conclusiones del Capítulo}

Es posible emplear la ondulación del geoide isostático sobre una estructura para inferir a partir de ella el balance isostático. Si esa condición es confirmada, es posible entonces validar las características corticales de la formación.

Analizando la estructura de Tandil en el sistema de Airy, el procedimiento empleado permite corroborar que la estructura estaría en equilibrio isostático y que los parámetros usados para el modelo teórico de corteza son los apropiados.

De esta manera se advierte que esta metodología puede complementar y validar el método tradicional que emplea anomalías de la gravedad. 


\section{Capítulo IX Conclusiones}

Con el presente trabajo se han identificado estructuras geológicas en un sector central de Argentina que comprende diversos escenarios topográficos: llanura el Este y zona central, montaña al Oeste y mar el extremo Sur-Este.

El estado isostático de estructuras geológicas se evaluó a partir del análisis de anomalías de aire libre y Bouguer. Estas anomalías gravimétricas se han calculado a partir de datos terrestres provenientes de la Red de Alta precisión del IGN, para toda el área de estudio.

Los datos gravimétricos terrestres utilizados están estandarizados en el sistema IGSN71, y han sido corregidos por el término de Honkasalo y procesados de acuerdo a las últimas normas para la reducción de los datos de gravedad (Hinze et al., 2005).

En el cálculo de las anomalías de aire libre se ha tenido en cuenta la corrección por la atmósfera y por altura. La corrección por altura, también conocida como corrección de aire libre, se obtuvo de acuerdo a las recomendaciones de las nuevas normas para la reducción de la gravedad usando el gradiente vertical de segundo orden de la gravedad de un modelo de Tierra elipsoidal en lugar de la aproximación de primer orden para una Tierra esférica usada tradicionalmente. La diferencia entre aplicar la corrección con una u otra aproximación es, en promedio, de $0.06 \mathrm{mGal}$ para los datos usados cuyas alturas varían entre 4 y $2200 \mathrm{~m}$. Para bajas elevaciones ( $<500 \mathrm{~m}$ ) ambas aproximaciones son consistentes entre sí, con diferencias menores a $0.1 \mathrm{mGal}$, sin embargo para mayores elevaciones la diferencia aumenta hasta $0.69 \mathrm{mGal}$ para alturas de $2200 \mathrm{~m}$.

La integración de anomalías de aire libre en el mar derivadas de la altimetría satelital del modelo DNSC08 permitió tener un mejor conocimiento e interpretación del campo de gravedad terrestre en términos de estructuras geológicas. Con la integración realizada se obtuvo una mejor representación de la cuenca de Claromecó al sur de la provincia de Buenos Aires, en la zona del Mar Argentino. 
Las anomalías de Bouguer remueven las masas topográficas existentes entre la estación y el geoide, para lo cual se utilizan diferentes aproximaciones sobre la forma, distribución y densidad de las masas topográfica. Se han usado dos aproximaciones para el cálculo de está anomalía: el modelo de lámina o placa plana de Bouguer y el modelo de capa esférica, obteniéndose anomalías de Bouguer planas y esféricas simples, respectivamente. Asimismo el efecto gravitacional de las masas topográficas circundantes al punto de cálculo no considerado por la placa de Bouguer ha sido considerado mediante la corrección topográfica, obteniéndose la anomalía de Bouguer plana completa. La corrección topográfica se obtuvo usando el programa Tc2DFT (Li, 1993) que usa la transformada de Fourier 2D utilizando una aproximación planar.

Se ha comparado la anomalía de Bouguer plana completa (ABPC) con las anomalías de Bouguer simples tanto en el modelo plano (ABPS) como esférico (ABES). Los resultados muestran que para zonas con cambios de elevación moderada, que corresponde a gran parte del área de trabajo, la elección de uno u otro enfoque es de menor importancia. Sin embargo en la zona de topografía movida (al Oeste de la zona de trabajo) se recomienda la selección de las anomalías de Bouguer planas completas.

En la confección de mapas de anomalías de Bouguer para la localización de estructuras locales en tierra se ha escogido la anomalía de Bouguer plana completa por ser la variable que toma en cuenta las anomalías de masa gravimétrica de acuerdo a las particularidades de la topografía. Para la identificación de estructuras en zona de mar, la situación es diferente, la corrección de Bouguer enmascara las estructuras locales. Por lo tanto se confeccionó un mapa de anomalías de Bouguer en tierra y anomalías de aire libre en el mar.

Para tener una evaluación de las anomalías de aire libre y Bouguer obtenidas se las comparó con el modelo geopotencial EGM2008. De la comparación se advierte un razonable ajuste, el $94 \%$ de los puntos evaluados (nodos de grilla) presentan diferencias de AAL menores que $\pm 30 \mathrm{mGal}$ y el $83 \%$ presentan diferencias de $\mathrm{AB}$ menores que $\pm 10 \mathrm{mGal}$.

Las anomalías de aire libre y Bouguer no pueden revelarnos el mecanismo de compensación (Pratt o Airy) pero la correlación que existe entre dichas anomalías y la topografía nos brinda información que sirve para un análisis a priori del estado isostático de la estructura. Del análisis cualitativo de las AAL y AB, se observa que en gran parte de la región (más del 80\%) los valores de AAL varían en un entorno de $\pm 20 \mathrm{mGal}$. En esta región los valores de $\mathrm{AB}$ presentan una imagen especular de la topografía, esto se advierte más claramente en el análisis por perfiles. Estos valores infieren a priori estructuras compensadas a escala regional. 
Valores positivos altos de AAL correlacionados con la topografía se muestran en el sector Oeste, a la altura del paralelo $-36^{\circ}$, que corresponden al Bordo Alto del Payún y Sierras de Chachauen, (provincia de Mendoza), con un pico de altura correspondiente al cerro Payún de 3680 m. Un comportamiento similar se advierte en las Sierra de Cochico y Cordillera del Viento (provincia de Neuquén). Estos valores mostrarían una estructura que no está en equilibrio isostático. Otro pico positivo de AAL se ubica al Este en el sistema de Tandil con valores de AB positivos, esta estructura no estaría compensada de acuerdo a los modelos isostáticos clásicos.

Los modelos geopotenciales brindan información del campo gravitacional terrestre global y pueden representarse como una descomposición espectral en longitudes de onda que permite analizar anomalías de masa a diferentes profundidades. Mediante el filtrado de la ondulación del geoide modelo EGM2008 se eliminó la componente de larga longitud de onda y se detectaron estructuras geológicas en la corteza para la zona de estudio. A partir de las estructuras identificadas y correlacionadas con mapas de anomalías de aire libre y Bouguer se infiere a priori el estado isostático de las mismas.

La estructura de Tandil muestra valores de anomalías de aire libre y Bouguer, que presumen un estado de descompensación de masas. Analizando la estructura a partir de las anomalías del geoide, en el sistema de Airy con parámetros de corteza normal establecidos para esa estructura por Del Cogliano (2006), se corroboró que los parámetros usados para el modelo teórico son los apropiados y que la estructura estaría en equilibrio isostático.

De esta manera se advierte que es posible emplear la ondulación del geoide isostático sobre una estructura para inferir a partir de ella el balance isostático y las características corticales. En consecuencia, esta metodología puede complementar y validar el método tradicional que emplea anomalías de la gravedad.

La metodología empleada resulta eficiente para la detección de estructuras geológicas en la corteza y el establecimiento a priori del estado isostático de las mismas. 


\section{Bibliografía Consultada y referencias citadas en el texto}

Andersen, O. B., and P. Knudsen (2009), DNSC08 mean sea surface and mean dynamic topography models, J. Geophys. Res., 114, C11001, doi:10.1029/2008JC005179.

Barthelmes, F. (2002) GFZ. Scientific Technical Reports STR 09/02. Definition of Functionals of the Geopotential and their calculation from Spherical Harmonics Models

Becker, J. J., D. T. Sandwell, W. H. F. Smith, J. Braud, B. Binder, J. Depner, D. Fabre, J. Factor, S. Ingalls, S-H. Kim, R. Ladner, K. Marks, S. Nelson, A. Pharaoh, R. Trimmer, J. Von Rosenberg, G. Wallace, P. Weatherall.,(2009). Global Bathymetry and Elevation Data at 30 Arc Seconds Resolution: SRTM30_PLUS, Marine Geodesy, 32:4, 355-371.

Brunini, C., G.Font, F.Galbán, E.Lauría, M.C.Pacino, R.Rodríguez, 2001. The Vertical Reference System in the Argentine Republic. Actas Symposium IAG.237-242

Cornaglia, L. (2007) La Sierra de San Luis, su estudio geofísico a partir de las ondulaciones del geoide. Entrega del premio “Ing. Eduardo E. Baglietto”. Anales Acad. Nac. de Ing. Buenos Aires, Tomo III:Pag.75-108.

Crovetto C., Molinari R. e Introcaso A. (2006). Aproximaciones para el cálculo del geoide isostático. Revista de la Asociación Geológica Argentina 61 (3):336-346.

Del Cogliano, D. (2006). Modelado del Geoide con GPS y Gravimetría. Caracterización de la estructura geológica de Tandil. Tesis Doctoral. FCEIyA - UNR.

Dermanis A. (1984). Kriging and collocation. A comparison. Manuscipta geodaetica. Vol 9. 159-167.

Dobrin, M.B. and Savit, C.H. (1988). Introduction to Geophysical Prospecting. Fourth Edition. McGraw-Hill. ISBN 10: 0070171963 ISBN 13: 978-0070171961.

Ecker E. and Mittermayer E. (1969). Gravity corrections for the influence of the atmosphere: Bulletin of Theoretical and Applied Geophysics, 70-80. 
Farr, T. G., P. A. Rosen, E. Caro, R. Crippen, R. Duren, S. Hensley, M. Kobrick, M. Paller, E. Rodriguez, L. Roth, D. Seal, S. Shaffer, J. Shimada, J. Umland, (2007).The Shuttle Radar Topography Mission, Rev. Geophys., 45, RG2004, doi: 10.1029/2005RG000183.

Forsberg R., (2003). An overview manual for the GRAVSOFT (Geodetic Gravity Field Modelling Programs), Kort og Matrikelstyrelsen, ed. DRAFT-1

Fowler C. M. R., (2005). The solid earth. An introduction to Global Geophysics. - Cambridge University Press. 2 edition -704 p.- ISBN 10: 52189307 0. ISBN 13: 978-0521893077.

Haxby W. and Turcotte D., (1978). On isostatic geoid anomalies. Journal Geophysical Journal Vol. 94. (B4)3876-3890

Hayford J.F. and Bowie W. (1912). The effect of topography and isostatic compensation upon the intensity of gravity. U. S. Coast Geod. Surv. Spec. Public. 10. 132 Pág.

Heiskanen W. and Moritz H. (1967) Physical Geodesy. W.H. Freeman and Company. San Fransisco and London.364 pág.

Heikkinen, M., (1979). On the Honkasalo term in tidal corrections to gravimetric observations, Bull.Geod., no. 53, p. 239-245.

Hinze W. J. et al., (2005). New standards for reducing gravity data: the North American gravity database, Geophysics, vol. 70, no 4, p. J25-J32.

Hinze W. J., (2003). Short note. Bouguer reduction density, why 2.67? , Geophysics, vol 68, no 5, p. 1559-1560.

Hofman B. and W. H. Moritz, (2005). Physical Geodesy, Springer Wien New York.

Honkasalo T., (1964). On the tidal gravity correction: Bulletin of Theoretical and Applied Geophysics, 6, 34-36.

IGM 130 IGN - 1879-2009. (2009) Instituto Geográfico Nacional. V.1. 144p. ISBN 978-98725448-2-9

Introcaso A. Pacino M.C. y Giuspi F. (1996). The Argentina-Chile Andes. Crustal thicknesses, isostasy, shortening and anomaly prediction from gravity studies. Third ISAG St. Malo (France). 63-66. 
Introcaso, A., (1997). Gravimetría. UNR Editora. Rosario. 355 pág.

Introcaso, A., (2003). Significativa descompensación isostática en la Cuenca del Colorado (República Argentina). Revista de la Asociación Geológica Argentina 58 (3): 474-478.

Introcaso, A. y Ruiz, F., (2005). El uso del geoide para determinar características corticales de una cuenca sedimentaria. Memorias del III Congreso de Geofísica. La Habana.

Introcaso, A. (2006). Geodesia Física. Boletín del Instituto de Fisiografía y Geología. Volumen Especial 1, Rosario. 128 pág. URL: http://www.fceia.unr.edu.ar/fisiografia.

Kuhn, M.; Featherstone, W. and Kirby J. - Complete spherical Bouguer gravity anomalies over Australia.(2009). Australian Journal of Earth Sciences. Vol 56 N² 2. P213-223

LaFehr, T. R., (1991). An exact solution for the gravity curvature, Bullard B, Geophysics, vol 56, no 8, August 1991.p.1179-1184.

Li, Yecai (1993). User’s Guide TOPOGEOP, Department of Geomatic Engineering University of Calgary, Alberta, CANADA.

Lillie, R. (1999). Whole earth geophysics. An introductory textbook for geologists and geophysisists. Prentice Hall. Upper Saddle River. New Jersey. 361 pág.

Mayer-Gürr, T. (2008) ITG-Grace03s www.geod.unibonn.de/itg-grace03.html.

Miranda, S. e Introcaso, A. (2000). Anomalías de ondulación del geoide isostático para los Andes Centrales de Bolovia en $20^{\circ}$ sur. Actas del vigésimo Congreso de la Asociación Argentina de Geofísicos y Geodestas. 207-211.

Mohr, P. J., and B. N. Taylor, 2001, The fundamental physical constant: Physics Today, 54, 616.

Pacino, M.C. and Tocho, C. (2009). Validation of EGM2008 over Argentina.- External quality evaluation reports of EGM08. Issue $\mathrm{n}^{\circ}$ 4. ISSN 1810-8555. 
Pavlis, N.K., Holmes, S.A., Kenyon, S.C. and Factor, J.K. (2008) An Earth Gravitational Model to degree 2160: EGM2008. Paper presented at the 2008 General Assembly of the European Geosciences Union, Vienna, Austria, April 13-18, 2008.

Pavlis, N. K., S. A. Holmes, S. Kenyon, and J. K. Factor (2012), The Development and Evaluation of the Earth Gravitational Model 2008 (EGM2008), J. Geophys. Res., doi:10.1029/2011JB008916, in press.

Pavlis N., Factor J., and Holmes S.(2005). Terrain-related gravimetric quantities computed for the next EGM.

Pedraza De Marchi ,A. C. y Tocho, C. (2010).Análisis de nuevos estándares para reducir datos de gravedad: aplicación en Tierra del Fuego. XXV Reunión Científica de la Asociación Argentina de Geofísicos y Geodesias. ISBN 978 - 987 - 25291-2-3. Pág. 239 - 240.

Pedraza De March,i A. C.; Tocho, C. y Ghidella M. (2010). Comparación de anomalías de gravedad derivadas de altimetría satelital con datos de gravedad marina en el margen continental argentino. XXV Reunión Científica de la Asociación Argentina de Geofísicos y Geodesias. ISBN 978 - 987 - 25291-2-3 Pág. 241

Rodriguez E., Morris C.S., Belz J.E., Chapin E.C., Martin J.M., Daffer W., and Hensley S. (2005). An Assessment of the SRTM Topographic Products. JPL. NASA. 143 pág.

Rummel, R. (2005). Geoid and Gravity in earth sciences - An overview. Text of book: Future Satellite Grvimetry and earth dynamics. Springer. ISBN 0-387-29796-0/ 978-0387-29796-5.

Sandwell D. and Smith W. (2009). Global marine gravity from retrcked Geosat and ERS-1 altimetry: Ridge segmentation versus spreading rate. Journal of geophysical research, Vo. 114, B01411, doi 1.1029/2008JB006008.

Talwani, M. (1998). Errors in the total Bouguer reduction. Short Note. Geophysics Vol. 63. P1125-1130.

Tapley B., Ries J., Bettadpur D., Chambers D., Cheng M., Condi F., Gunter B., Kang Z., Nagel P., Pastor R. Pekker T., Poole S., and Wang F. (2005). GGM02 - An improved Earth gravity field model from GRACE. Journal of geodesy. Doi 10.1007/s00190-005-0480-z. 
Tocho, C. (2006). A gravimetric geoid modelling. Tesis Doctoral. Facultad de Ciencias Astronómicas y Geofísicas. Universidad Nacional de La Plata.

Tocho, C. y Pacino M.C. (2009). Evaluación del modelo geopotencial terrestre EGM 2008 en Argentina. XXIV Reunión Científica AAGG 2009.

Torge W., (1989). Gravimetry, Walter de Gruyter Berlin, New York.- 465 pág. ISBN 3-11010702-3.

Torge, W. (2001). Geodesy. 3rd Edition.Walter de Gruyter. Berlin - New York.255 pág.

Turcotte D. and Schubert G. (2002).Geodynamics. Cambridge University Press. 2 edition 472 pág.- ISBN 10: 521 666244. ISBN 13: 978-0521666244.

Uotila U.A. (1980) Note to users of International Gravity standardization net 1971: Journal of geodesy, 54, 407-408.

Villella J.C. y Pacino M.C. (2010). Interpolación gravimétrica para el cálculo de los números geopotenciales de la red altimétrica de Argentina en zonas de alta montaña. Geoacta 35, $13-$ 26. ISSN 1852-7744.

Wenzel H., (1985). Hochauflosende fur des gravitationspotential der erde [1]: Wissenschaftliche arbeiten del Fachrichtung Vermessungswesen der Universitat Hannover, 137.

http://icgem.gfz-potsdam.de/ICGEM/ICGEM.html 


\section{Anexo 1}

\section{A.1.- Parámetros del elipsoide GRS 80}

En esta Tesis se usó el elipsoide GRS 80. Los parámetros del elipsoide GRS80 son los siguientes: el semieje mayor a es de $6.378 .137 \mathrm{~m}$; el semieje-menor b es $6.356 .752,3141 \mathrm{~m}$; el aplastamiento $\mathbf{f}$ es $0.003352810681 ; \mathbf{g}_{\mathbf{e}}$ es $9.7803267715 \mathrm{~m} / \mathrm{s}^{2} ;$ y $\mathbf{m}$ es $\omega^{2} \mathrm{a}^{2} \mathrm{~b}^{2} / \mathrm{GM}$, con un valor de 0.00344978600308 , donde $\omega$ es la velocidad angular $\left(7292115 \times 10^{-11}\right.$ radianes/s) y GM es la constante gravitacional geocéntrica $\left(3986005 \times 108 \mathrm{~m}^{3} / \mathrm{s}^{2}\right)$.

A.2.- La fórmula exacta para el cálculo de los coeficientes $\mu$ y $\lambda$ de la corrección de

\section{Bullard B}

La corrección de Bullard B o corrección esférica (Figura A.1), propuesta por Bullard en 1936, tiene el propósito de convertir la geometría de la lámina plana infinita de Bouguer a una capa esférica.

La fórmula exacta (LaFehr, 1991) para el cálculo de los coeficientes $\mu$ y $\lambda$ de la ecuación (2.17) se muestra en las ecuaciones (A.1), donde $\mathrm{S}$ es el radio de la capa esférica y se da un valor de $166,735 \mathrm{~km}$, el espesor de la capa $\mathrm{H}$ (altura de la estación) y en radio medio $\mathrm{R}_{0}$ de la tierra que se toma de $6371 \mathrm{~km}$.

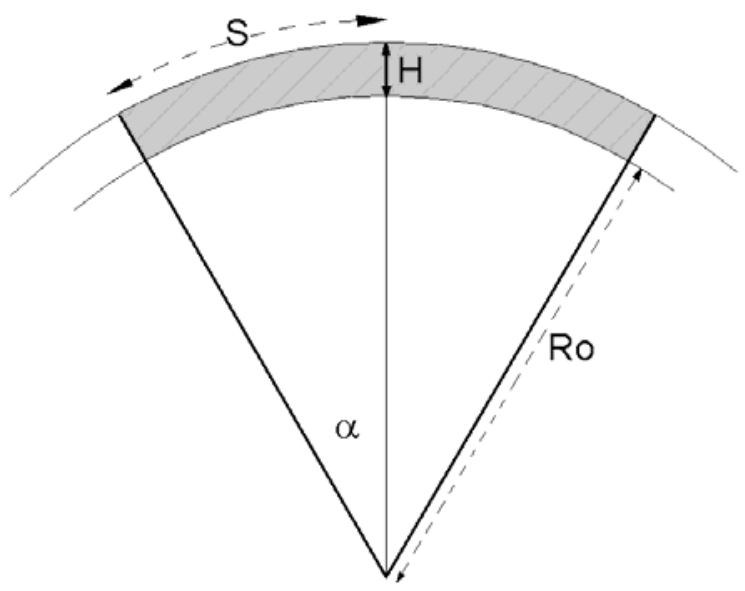

Figura A.1 -Corrección de Bullard B.

$$
R=R_{0}+H \quad \alpha=\frac{S}{R} \quad \delta=\frac{R_{0}}{R} \quad \eta=\frac{H}{R} \quad \mu=\frac{1}{3} \eta^{2}-\eta
$$


donde

$$
\lambda=\frac{1}{3}\left\{\left(d+f \delta+\delta^{2}\right)\left[(f-\delta)^{2}+k\right]^{1 / 2}+p+m \cdot \log _{e} \frac{n}{f-\delta+\left[(f-\delta)^{2}+k\right]^{1 / 2}}\right\}
$$

$d=3 \cos ^{2} \alpha-2$

$f=\cos \alpha$

$k=\operatorname{sen}^{2} \alpha$

$p=-6 \cos ^{2} \alpha \cdot \operatorname{sen}(\alpha / 2)+4 \operatorname{sen}^{3}(\alpha / 2)$

$m=-3 \operatorname{sen}^{2} \alpha \cos \alpha$

$n=2\left[\operatorname{sen}(\alpha / 2)-\operatorname{sen}^{2}(\alpha / 2)\right]$ 